\title{
CHARACTER TABLE OF A BOREL SUBGROUP OF THE REE GROUPS ${ }^{2} F_{4}\left(q^{2}\right)$
}

\author{
FRANK HIMSTEDT AND SHIH-CHANG HUANG
}

\begin{abstract}
We compute the conjugacy classes and character table of a Borel subgroup of the Ree groups ${ }^{2} F_{4}\left(2^{2 n+1}\right)$ for all $n \geqslant 1$ and prove that these Borel subgroups are $M$-groups. We determine the degrees of the irreducible characters of the Sylow-2-subgroups of ${ }^{2} F_{4}\left(2^{2 n+1}\right)$ and show that the IsaacsMalle-Navarro version of the McKay conjecture holds for ${ }^{2} F_{4}\left(2^{2 n+1}\right)$ in characteristic 2 . For most of the calculations we use CHEVIE.
\end{abstract}

\section{Introduction}

Let ${ }^{2} F_{4}\left(q^{2}\right)$ be the simple Ree group with $q^{2}=2^{2 n+1}$ and $n$ a positive integer. The character table of ${ }^{2} F_{4}\left(q^{2}\right)$ is known by work of $\mathrm{G}$. Malle, see [16] and the character table in CHEVIE [8].

In this paper we compute the (complex) irreducible characters of a Borel subgroup $B$ of ${ }^{2} F_{4}\left(q^{2}\right)$. Some of our methods are similar to those used by H. Enomoto and H. Yamada in [5]. We construct most of the irreducible characters of $B$ by inducing linear characters from suitable subgroups. To calculate the values of the remaining irreducible characters we use tensor products of characters, restrictions of unipotent characters of ${ }^{2} F_{4}\left(q^{2}\right)$ to $B$ and orthogonality relations.

We consider the character table of $B$ as a starting point for the construction of the irreducible characters of the maximal parabolic subgroups of ${ }^{2} F_{4}\left(q^{2}\right)$. These characters might be helpful in getting new information on the decomposition numbers and the degrees of low-dimensional representations of ${ }^{2} F_{4}\left(q^{2}\right)$ in non-defining characteristic along the same line as in [9] or [21].

We have implemented the character table of $B$ as a generic character table in the MAPLE [4] part of CHEVIE [8] and we use MAPLE-programs for restricting and inducing class functions. The use of CHEVIE allows us to easily compute scalar products of class functions and provides tests for the obtained character tables. For calculations with elements of ${ }^{2} F_{4}\left(q^{2}\right)$, we use computer programs written by C. Köhler and the first author in GAP [6].

In [20], B. Szegedy has shown that the Borel subgroups of most of the classical groups over finite fields of odd characteristic are $M$-groups, that means, all irreducible characters can be obtained by induction from linear characters of suitable subgroups. Our constructions of the irreducible characters of $B$ imply that the same is true for the Borel subgroups of ${ }^{2} F_{4}\left(q^{2}\right)$. 
As an application of the character table of $B$ we determine the degrees of the irreducible characters of a Sylow-2-subgroup $U$ of ${ }^{2} F_{4}\left(q^{2}\right)$ and for every degree we compute the number of irreducible characters of $U$ with this degree.

Finally, we consider the McKay conjecture for ${ }^{2} F_{4}\left(q^{2}\right)$. In [13] Isaacs, Malle and Navarro reduce the McKay conjecture to a question about finite simple groups: They show that every finite group will satisfy the McKay conjecture if every finite non-abelian simple group is good. By counting characters of $B$ and ${ }^{2} F_{4}\left(q^{2}\right)$ which are fixed by certain automorphisms, we prove that the groups ${ }^{2} F_{4}\left(q^{2}\right)$ are good for the prime 2 .

This paper is organized as follows: In Sections 2 and 3, we fix notation, state some properties of ${ }^{2} F_{4}\left(q^{2}\right)$ and give a short description of some conjugacy classes of ${ }^{2} F_{4}\left(q^{2}\right)$. In Section 4, we determine the character table of the Borel subgroup $B$. In Section 5, we prove that $B$ is an $M$-group and determine the degrees of the irreducible characters of the Sylow-2-subgroup $U$. Section 6 deals with the McKay conjecture for ${ }^{2} F_{4}\left(q^{2}\right)$ in characteristic 2. Details on conjugacy classes and character tables are given in an appendix. A CHEVIE file with the generic character table of $B$ is available from http://www. Ims.ac.uk/jcm/12/lms2008-001/appendix-a/.

\section{Notation and group-theoretical properties of ${ }^{2} F_{4}\left(q^{2}\right)$}

We choose the notation similar to that in [17] and [19]. Let $V$ be a Euclidean vector space with scalar product $(\cdot, \cdot)$, let $\left\{e_{1}, e_{2}, e_{3}, e_{4}\right\}$ be an orthonormal basis of $V$ and let $\Phi$ be the set consisting of the 48 vectors

$$
e_{i}, e_{i}+e_{j}, \frac{1}{2}\left(e_{i}+e_{j}+e_{k}+e_{l}\right)
$$

where $i, j, k, l \in\{ \pm 1, \pm 2, \pm 3, \pm 4\},|i|,|j|,|k|,|l|$ are different and $e_{-i}=-e_{i}$ for all $i$. The set $\Phi$ is a root system of type $F_{4}$ and the set $\Delta:=\left\{r_{1}, r_{2}, r_{3}, r_{4}\right\}$ with the simple roots

$$
r_{1}:=e_{2}-e_{3}, r_{2}:=e_{3}-e_{4}, r_{3}:=e_{4}, r_{4}:=\frac{1}{2}\left(e_{1}-e_{2}-e_{3}-e_{4}\right)
$$

is a basis of $\Phi$. We fix a total ordering on $V$ (and hence $\Phi$ ) as in $[\mathbf{1 7},(3.1)]$. The positive roots are the simple roots $r_{1}, r_{2}, r_{3}, r_{4}$ and

$$
\begin{gathered}
r_{5}:=r_{1}+r_{2}, r_{6}:=r_{2}+r_{3}, r_{7}:=r_{3}+r_{4}, r_{8}:=r_{1}+r_{2}+r_{3}, r_{9}:=r_{2}+2 r_{3}, \\
r_{10}:=r_{2}+r_{3}+r_{4}, r_{11}:=r_{1}+r_{2}+2 r_{3}, r_{12}:=r_{1}+r_{2}+r_{3}+r_{4}, \\
r_{13}:=r_{2}+2 r_{3}+r_{4}, r_{14}:=r_{1}+2 r_{2}+2 r_{3}, r_{15}:=r_{1}+r_{2}+2 r_{3}+r_{4}, \\
r_{16}:=r_{2}+2 r_{3}+2 r_{4}, r_{17}:=r_{1}+2 r_{2}+2 r_{3}+r_{4}, r_{18}:=r_{1}+r_{2}+2 r_{3}+2 r_{4}, \\
r_{19}:=r_{1}+2 r_{2}+3 r_{3}+r_{4}, r_{20}:=r_{1}+2 r_{2}+2 r_{3}+2 r_{4}, \\
r_{21}:=r_{1}+2 r_{2}+3 r_{3}+2 r_{4}, r_{22}:=r_{1}+2 r_{2}+4 r_{3}+2 r_{4}, \\
r_{23}:=r_{1}+3 r_{2}+4 r_{3}+2 r_{4}, r_{24}:=2 r_{1}+3 r_{2}+4 r_{3}+2 r_{4} .
\end{gathered}
$$

Let $L$ be a simple complex Lie algebra of type $F_{4}$ and let $\left\{h_{r} \mid r \in \Delta\right\} \cup\left\{e_{r} \mid r \in \Phi\right\}$ be a Chevalley basis of $L$ (in the sense of [2, Theorem 4.2.1]).

We fix an integer $n>0$ and set $\theta=2^{n}$ and $q:=\sqrt{2^{2 n+1}} \in \mathbb{R}_{>0}$. Let $\mathbb{F}_{q^{2}}$ be a finite field with $q^{2}$ elements, $\mathbb{F}$ its algebraic closure and $\mathbf{G}=\left\langle x_{r}(t) \mid r \in \Phi, t \in \mathbb{F}\right\rangle$ the Chevalley group over the field $\mathbb{F}$ constructed from the Lie algebra $L$. So $\mathbf{G}$ is 
a simple simply connected algebraic group of Dynkin type $F_{4}$, defined over $\mathbb{F}_{q^{2}}$. For $r \in \Phi$ let $\lambda(r):=(r, r)$ be the length of $r$. Let $r \mapsto \bar{r}$ be the permutation of order 2 of $\Phi$ as in $[\mathbf{1 7},(1.4)]$. By [17, Theorem 2.7], there is a map $F: \mathbf{G} \rightarrow \mathbf{G}$ sending $x_{r}(t)$ to $x_{\bar{r}}\left(t^{\lambda(\bar{r}) \theta}\right)$ and we get $\mathbf{G}^{F}={ }^{2} F_{4}\left(q^{2}\right)$. We will consider the following $F$-stable elements of $\mathbf{G}$ (see [17, p. 407]):

$$
\begin{aligned}
\alpha_{1}(t) & :=x_{3}\left(t^{\theta}\right) x_{2}(t) x_{6}\left(t^{\theta+1}\right), & \alpha_{7}(t) & :=x_{13}\left(t^{\theta}\right) x_{14}(t), \\
\alpha_{2}(t) & :=x_{6}\left(t^{\theta}\right) x_{9}(t), & \alpha_{8}(t) & :=x_{12}\left(t^{\theta}\right) x_{18}(t), \\
\alpha_{3}(t) & :=x_{4}\left(t^{\theta}\right) x_{1}(t), & \alpha_{9}(t) & :=x_{15}\left(t^{\theta}\right) x_{20}(t), \\
\alpha_{4}(t) & :=x_{7}\left(t^{\theta}\right) x_{5}(t) x_{12}\left(t^{\theta+1}\right), & \alpha_{10}(t) & :=x_{17}\left(t^{\theta}\right) x_{22}(t), \\
\alpha_{5}(t) & :=x_{8}\left(t^{\theta}\right) x_{16}(t) x_{21}\left(t^{\theta+1}\right), & \alpha_{11}(t) & :=x_{19}\left(t^{\theta}\right) x_{23}(t), \\
\alpha_{6}(t) & :=x_{10}\left(t^{\theta}\right) x_{11}(t) x_{19}\left(t^{\theta+1}\right), & \alpha_{12}(t) & :=x_{21}\left(t^{\theta}\right) x_{24}(t)
\end{aligned}
$$

with $t \in \mathbb{F}_{q^{2}}$ (here and in the following, we write $x_{i}(t)$ for $x_{r_{i}}(t)$ ). For group elements $x, y$ let $[x, y]:=x^{-1} y^{-1} x y$. The commutator relations are given in Table 1 where $j(i)=2,8,12,11$ corresponding to $i=1,4,5,6$ respectively.

Table 1 (Shinoda $[\mathbf{1 8},(2.3)])$ : Commutator relations in ${ }^{2} F_{4}\left(q^{2}\right)$.

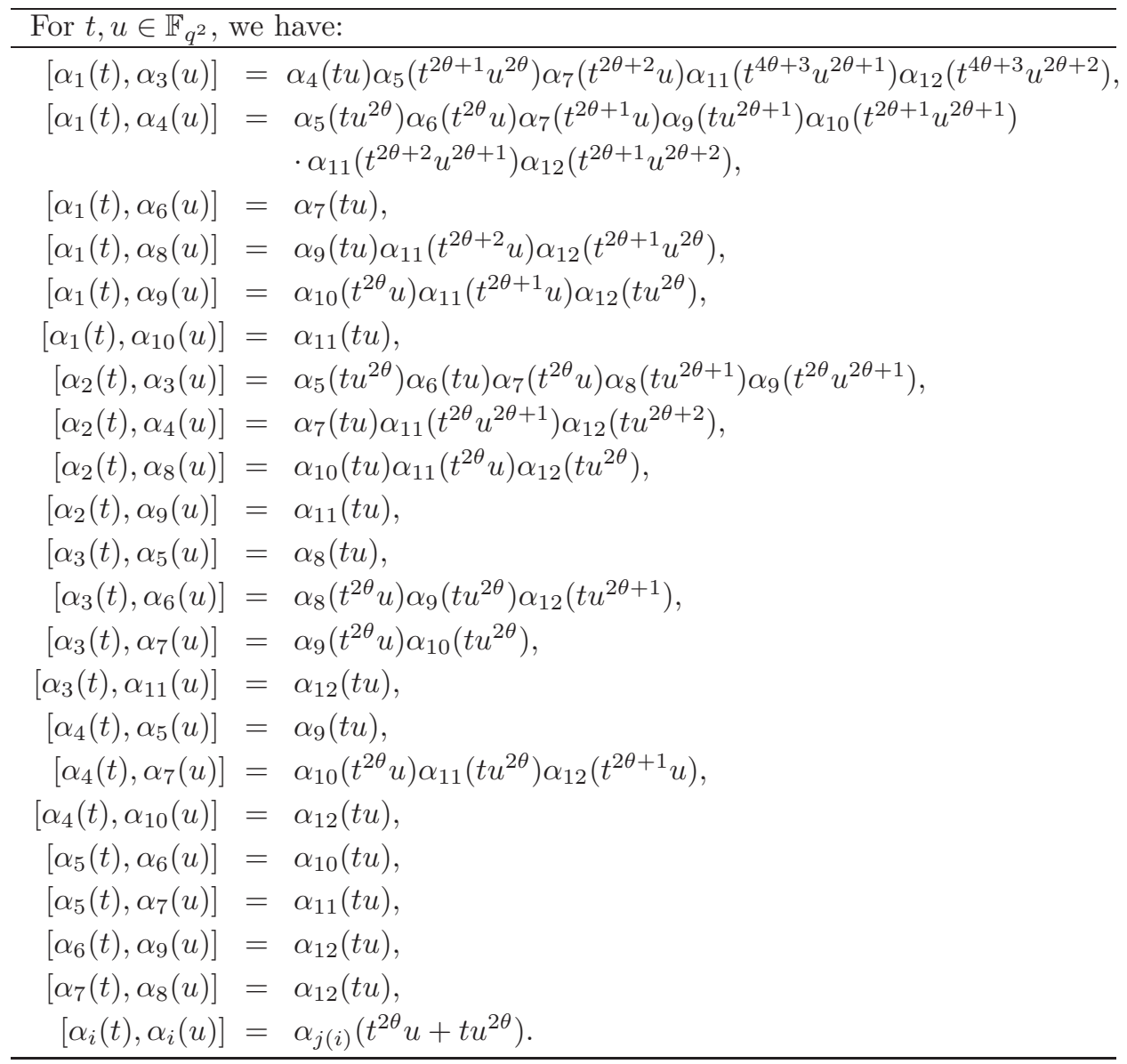


Note that the relation for $\left[\alpha_{2}(t), \alpha_{3}(u)\right]$ in $[18,(2.3)]$ contains a mistake. All commutators $\left[\alpha_{i}(t), \alpha_{j}(u)\right]$ not listed in Table 1 are equal to 1 . Furthermore, for $i=1,4,5,6$ we have

$$
\alpha_{i}(t) \alpha_{i}(u)=\alpha_{i}(u+t) \alpha_{j(i)}\left(t u^{2 \theta}\right) .
$$

For any field $K$, we denote its multiplicative group by $K^{\times}$. Fix an $F$-stable maximal torus $\mathbf{T}$ of $\mathbf{G}$, normalizing all root subgroups $X_{r}=\left\{x_{r}(t) \mid t \in \mathbb{F}\right\}$ of $\mathbf{G}$. We parametrize the elements of $\mathbf{T}$ in the same way as in $[19,(1.4)]$ : Let $X:=\mathbb{Z} \Phi$ be the root lattice of $\mathbf{G}$ with respect to $\mathbf{T}$. There is a natural isomorphism between the abelian groups $\mathbf{T}$ and $\operatorname{Hom}\left(X, \mathbb{F}^{\times}\right)$(see $[\mathbf{3}$, Section 1.11 and Proposition $3.1 .2(\mathrm{i})])$ and we write $h\left(z_{1}, z_{2}, z_{3}, z_{4}\right)$ for the element of $\mathbf{T}$ corresponding to $\chi \in \operatorname{Hom}\left(X, \mathbb{F}^{\times}\right)$with $\chi\left(e_{i}\right)=z_{i}(i=1,2,3,4)$. We mention that there is an alternative parametrization of the elements of $\mathbf{T}$ (which in fact we use for our GAP-programs): Let $\left\{\gamma_{1}, \gamma_{2}, \gamma_{3}, \gamma_{4}\right\}$ be a $\mathbb{Z}$-basis of the cocharacter group $Y$ dual to $\Delta$. By [3, Proposition 3.1.2 (ii)], we have $\mathbf{T} \cong Y \otimes \mathbb{F}^{\times}$as abelian groups. Every element of $Y \otimes \mathbb{F}^{\times}$can be written uniquely as $\sum_{i=1}^{4} \gamma_{i} \otimes \lambda_{i}$ with $\lambda_{i} \in \mathbb{F}^{\times}$and we write $\left(\lambda_{1}, \lambda_{2}, \lambda_{3}, \lambda_{4}\right)$ for the corresponding element of $\mathbf{T}$. The maps transforming one parametrization into the other are given by:

$$
\begin{aligned}
\left(\lambda_{1}, \lambda_{2}, \lambda_{3}, \lambda_{4}\right) & \mapsto h\left(\lambda_{1} \lambda_{2}^{2} \lambda_{3}^{3} \lambda_{4}^{2}, \lambda_{1} \lambda_{2} \lambda_{3}, \lambda_{2} \lambda_{3}, \lambda_{3}\right) \text { and } \\
h\left(z_{1}, z_{2}, z_{3}, z_{4}\right) & \mapsto\left(z_{2} z_{3}^{-1}, z_{3} z_{4}^{-1}, z_{4},\left(z_{1} z_{2}^{-1} z_{3}^{-1} z_{4}^{-1}\right)^{1 / 2}\right) .
\end{aligned}
$$

The action of $F$ on $\mathbf{T}$ is given by $F\left(h\left(z_{1}, z_{2}, z_{3}, z_{4}\right)\right)=h\left(z_{1}^{\theta} z_{2}^{\theta}, z_{1}^{\theta} z_{2}^{-\theta}, z_{3}^{\theta} z_{4}^{\theta}, z_{3}^{\theta} z_{4}^{-\theta}\right)$ and $\mathbf{T}^{F}$ consists precisely of the elements of the form $h\left(z_{1}, z_{1}^{2 \theta-1}, z_{2}, z_{2}^{2 \theta-1}\right)$ with $z_{i} \in \mathbb{F}_{q^{2}}^{\times}($see $[19,(1.4)$ and $(3.1)])$. As in [18] we usually only write $h\left(z_{1}, z_{2}\right)$ instead of $h\left(z_{1}, z_{1}^{2 \theta-1}, z_{2}, z_{2}^{2 \theta-1}\right)$.

Table 2 (Shinoda $[\mathbf{1 8},(2.4)])$ : Action of $\mathbf{T}^{F}$ on the root subgroups.

\begin{aligned} & \hline For $z_{1}, z_{2} \in \mathbb{F}_{q^{2}}^{\times}, u \in \mathbb{F}_{q^{2}}$, we have: \\ & \hline$h\left(z_{1}, z_{2}\right) \cdot \alpha_{1}(u) \cdot h\left(z_{1}, z_{2}\right)^{-1}=\alpha_{1}\left(z_{2}^{2-2 \theta} u\right) \\ &, h\left(z_{1}, z_{2}\right) \cdot \alpha_{2}(u) \cdot h\left(z_{1}, z_{2}\right)^{-1}=\alpha_{2}\left(z_{2}^{2 \theta} u\right),=\alpha_{3}\left(z_{1}^{2 \theta-1} z_{2}^{-1} u\right) \\ &, h\left(z_{1}, z_{2}\right) \cdot \alpha_{3}(u) \cdot h\left(z_{1}, z_{2}\right)^{-1}=\alpha_{4}\left(z_{1}^{2 \theta-1} z_{2}^{1-2 \theta} u\right) \\ &, h\left(z_{1}, z_{2}\right) \cdot \alpha_{4}(u) \cdot h\left(z_{1}, z_{2}\right)^{-1}=\alpha_{5}\left(z_{1}^{2-2 \theta} u\right) \\ &, h\left(z_{1}, z_{2}\right) \cdot \alpha_{5}(u) \cdot h\left(z_{1}, z_{2}\right)^{-1}=\alpha_{6}\left(z_{1}^{2 \theta-1} z_{2}^{2 \theta-1} u\right) \\ &, h\left(z_{1}, z_{2}\right) \cdot \alpha_{6}(u) \cdot h\left(z_{1}, z_{2}\right)^{-1}=\alpha_{7}\left(z_{1}^{2 \theta-1} z_{2} u\right) \\ &, h\left(z_{1}, z_{2}\right) \cdot \alpha_{7}(u) \cdot h\left(z_{1}, z_{2}\right)^{-1}=\alpha_{8}\left(z_{1} z_{2}^{-1} u\right) \\ &\left.,h\left(z_{1}, z_{2}\right) \cdot \alpha_{8}(u) \cdot h\left(z_{1}, z_{2}\right)^{-1}=z_{1} z_{2}^{1-2 \theta} u\right) \\ &\left.,h\left(z_{1}, z_{2}\right) \cdot \alpha_{9}(u) \cdot h\left(z_{1}, z_{2}\right)^{-1}=\alpha_{11} z_{2}^{2 \theta-1} u\right) \\ &\left.,h\left(z_{1}, z_{2}\right) \cdot \alpha_{10}(u) \cdot h\left(z_{1}, z_{2}\right)^{-1}=z_{2} u\right) \\ &, h\left(z_{1}, z_{2}\right) \cdot \alpha_{11}(u) \cdot h\left(z_{1}, z_{2}\right)^{-1}=\alpha_{12}\left(z_{1}^{2 \theta} u\right) \\ &. h\left(z_{1}, z_{2}\right) \cdot \alpha_{12}(u) \cdot h\left(z_{1}, z_{2}\right)^{-1}=\end{aligned}$

For $r \in \Phi$, let $n_{r}$ be the element in $\mathbf{N}:=N_{\mathbf{G}}(\mathbf{T})$, given by [2, Lemma 6.4.4]. Then $\mathbf{N}$ is generated by $\mathbf{T}$ and the elements $n_{r}$ for $r \in \Delta$. We have a canonical homomorphism with kernel $\mathbf{T}$ from $\mathbf{N}$ onto $\mathbf{W}$, the Weyl group of the root system $\Phi$, mapping $n_{r}$ to the reflection $w_{r}$ of $V$ at the hyperplane orthogonal to $r$. This allows 
us to identify $\mathbf{W}$ with $\mathbf{N} / \mathbf{T}$. As $\mathbf{N}$ and $\mathbf{T}$ are $F$-stable, this homomorphism induces an action of $F$ on $\mathbf{W}$. Define $n_{a}:=n_{r_{1}} n_{r_{4}}$ and $n_{b}:=n_{r_{6}} n_{r_{3}}=n_{r_{2}} n_{r_{3}} n_{r_{2}} n_{r_{3}}$. Then $\mathbf{N}^{F}$ is generated by $\mathbf{T}^{F}$ and the elements $n_{a}$ and $n_{b}$. The factor group $\mathbf{W}^{1}:=$ $\mathbf{N}^{F} / \mathbf{T}^{F}$ is isomorphic to a dihedral group of order 16 .

We have $n_{a} \alpha_{i}(t) n_{a}^{-1}=\alpha_{n_{a}(i)}(t)$ and $n_{b} \alpha_{i}(t) n_{b}^{-1}=\alpha_{n_{b}(i)}(t)$ where $n_{a}(i)$ and $n_{b}(i)$ are defined by Table 3 , see also $[18,(2.2)]$. Note that for $t \neq 0$, the elements $n_{a} \cdot \alpha_{3}(t) \cdot n_{a}^{-1}, n_{b} \cdot \alpha_{1}(t) \cdot n_{b}^{-1}$ and $n_{b} \cdot \alpha_{2}(t) \cdot n_{b}^{-1}$ cannot be written as a product of the $\alpha_{i}(i=1,2, \ldots, 12)$.

Table 3: Action of $\mathbf{W}^{1}$ on the root subgroups.

\begin{tabular}{c|rrrrrrrrrrrr}
\hline$i$ & 1 & 2 & 3 & 4 & 5 & 6 & 7 & 8 & 9 & 10 & 11 & 12 \\
\hline$n_{a}(i)$ & 4 & 8 & & 1 & 6 & 5 & 9 & 2 & 7 & 10 & 12 & 11 \\
$n_{b}(i)$ & & & 7 & 6 & 5 & 4 & 3 & 11 & 10 & 9 & 8 & 12 \\
\hline
\end{tabular}

The action of $\mathbf{W}$ on $\mathbf{T}$ is given by Table 4 .

Table 4: Action of $\mathbf{W}$ on $\mathbf{T}$.

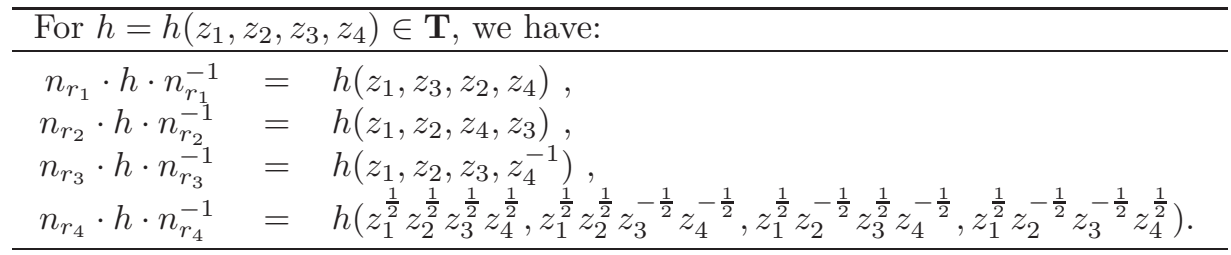

In particular: $n_{a} \cdot h\left(z_{1}, z_{2}\right) \cdot n_{a}^{-1}=h\left(z_{1}^{\theta} z_{2}^{\theta}, z_{1}^{\theta} z_{2}^{-\theta}\right)$ and $n_{b} \cdot h\left(z_{1}, z_{2}\right) \cdot n_{b}^{-1}=h\left(z_{1}, z_{2}^{-1}\right)$ (see also $[18,(2.4)])$.

Following [15], we fix some notation in Table 5 to parametrize conjugacy classes and characters: Let $\mathbb{Q}_{p^{\prime}}$ be the additive group of all rational numbers with a denominator not divisible by $p$. We fix an isomorphism of groups $\varphi_{1}: \mathbb{Q}_{p^{\prime}} / \mathbb{Z} \rightarrow \mathbb{F}^{\times}$as in $\left[3\right.$, Proposition 3.1.3], and the embedding $\varphi_{2}: \mathbb{Q}_{p^{\prime}} / \mathbb{Z} \hookrightarrow \mathbb{C}^{\times}, \frac{r}{s} \mapsto e^{2 \pi i r / s}$. (We describe the elements of $\mathbb{Q}_{p^{\prime}} / \mathbb{Z}$ via representatives $\frac{r}{s}$ in $\left.\mathbb{Q}_{p^{\prime}}(r, s \in \mathbb{Z}, p \nmid s)\right)$. Let $\phi_{n}$ denote the $n$th cyclotomic polynomial in $q$, for example: $\phi_{1}=q-1, \phi_{2}=q+1$, $\phi_{12}=q^{4}-q^{2}+1$.

For any finite group $H$ let $(\cdot, \cdot)_{H}$ be the usual scalar product on the space of class functions of $H$ and let $\operatorname{Irr}(H)$ be the set of (complex) irreducible characters of $H$. We denote the trivial character of $H$ by $\mathbf{1}_{H}$ or $\mathbf{1}$. If $\chi$ is a character of a subgroup $H_{1}$ of $H$ we write $\chi^{H}$ for the induced character, and if $\chi$ is a character of $H$ we write $\chi_{H_{1}}$ for the restriction of $\chi$ to the subgroup $H_{1}$ of $H$.

\section{The conjugacy classes of ${ }^{2} F_{4}\left(q^{2}\right)$}

The conjugacy classes of $\mathbf{G}^{F}={ }^{2} F_{4}\left(q^{2}\right)$ were determined by K. Shinoda in [19]. To fix notation and because we will use these conjugacy classes when restricting 
Table 5: Notation for generic roots of unity.

\begin{tabular}{c|cc}
\hline$\mu \in \mathbb{Q}_{p^{\prime}} / \mathbb{Z}$ & $\varphi_{1}(\mu) \in \mathbb{F}^{\times}$ & $\varphi_{2}(\mu) \in \mathbb{C}^{\times}$ \\
\hline$\frac{1}{3}$ & $\tilde{\varepsilon}_{3}$ & $\varepsilon_{3}$ \\
$\frac{1}{4}$ & $\tilde{\varepsilon}_{4}$ & $\varepsilon_{4}$ \\
$\frac{1}{q^{2}+\sqrt{2} q+1}$ & $\tilde{\varphi}_{8}^{\prime}$ & $\varphi_{8}^{\prime}$ \\
$\frac{1}{q^{2}-\sqrt{2} q+1}$ & $\tilde{\varphi}_{8}^{\prime \prime}$ & $\varphi_{8}^{\prime \prime}$ \\
$\frac{1}{\left(q^{2}+\sqrt{2} q+1\right)\left(q^{2}-1\right)}$ & $\tilde{\psi}_{8}^{\prime}$ & $\psi_{8}^{\prime}$ \\
$\frac{1}{\left(q^{2}-\sqrt{2} q+1\right)\left(q^{2}-1\right)}$ & $\tilde{\psi}_{8}^{\prime \prime}$ & $\psi_{8}^{\prime \prime}$ \\
$\frac{1}{q^{4}+\sqrt{2} q^{3}+q^{2}+\sqrt{2} q+1}$ & $\tilde{\varphi}_{24}^{\prime}$ & $\varphi_{24}^{\prime}$ \\
$\frac{1}{q^{4}-\sqrt{2} q^{3}+q^{2}-\sqrt{2} q+1}$ & $\tilde{\varphi}_{24}^{\prime \prime}$ & $\varphi_{24}^{\prime \prime}$ \\
$\frac{1}{q^{n}-1}$ & $\tilde{\zeta}_{n}$ & $\zeta_{n}$ \\
$\frac{1}{q^{n}+1}$ & $\tilde{\xi}_{n}$ & $\xi_{n}$ \\
$\frac{1}{\phi_{n}}$ & $\tilde{\varphi}_{n}$ & $\varphi_{n}$ \\
\hline
\end{tabular}

characters from ${ }^{2} F_{4}\left(q^{2}\right)$, we give a short description of these classes.

We call semisimple elements $s_{1}, s_{2} \in \mathbf{G}^{F}$ equivalent if and only if their centralizers $C_{\mathbf{G}}\left(s_{1}\right)$ and $C_{\mathbf{G}}\left(s_{2}\right)$ are $\mathbf{G}^{F}$-conjugate. Let $s_{1}, s_{2}$ be elements which are equivalent to each other. After conjugation in $\mathbf{G}^{F}$ we can suppose $C_{\mathbf{G}}\left(s_{1}\right)=C_{\mathbf{G}}\left(s_{2}\right)=$ : $C$. Then, $s_{1}$ and $s_{2}$ are contained in all maximal tori of $C$. Hence, we may always assume that two equivalent semisimple elements are contained in the same $F$-stable maximal torus of $\mathbf{G}$.

For each $F$-stable maximal torus $\tilde{\mathbf{T}}$ of $\mathbf{G}$ there is $g \in \mathbf{G}$ such that $\tilde{\mathbf{T}}^{g}=\mathbf{T}$ and $n:=g^{-1} F(g) \in \mathbf{N}=N_{\mathbf{G}}(\mathbf{T})$. The action of $F$ on $\tilde{\mathbf{T}}$ corresponds to the action of the $\operatorname{map}\left(F n^{-1}\right): \mathbf{G} \rightarrow \mathbf{G}, x \mapsto{ }^{n} F(x)$ on $\mathbf{T}$ (i.e. $F(\tilde{t})^{g}={ }^{n} F\left(\tilde{t}^{g}\right)$ for all $\tilde{t} \in \tilde{\mathbf{T}}$ ) and this action is already determined by $w:=n \mathbf{T} \in \mathbf{W}=N(\mathbf{T}) / \mathbf{T}$, so that we can write $\left(F w^{-1}\right): \mathbf{T} \rightarrow \mathbf{T}, t \mapsto{ }^{w} F(t)$. The $\mathbf{G}^{F}$-classes of $F$-stable maximal tori of $\mathbf{G}$ are parametrized by the $F$-conjugacy classes of $\mathbf{W}$ (cf. [3, Proposition 3.3.3]). Thus, we can describe the $\mathbf{G}^{F}$-classes of maximal tori of $\mathbf{G}^{F}$ by the sets $T_{j}:=\mathbf{T}^{\left(F w_{j}^{-1}\right)}$ of fixed points of $\left(F w_{j}^{-1}\right)$ on $\mathbf{T}$, where $w_{j}$ runs over a set of representatives for the $F$-conjugacy classes of $\mathbf{W}$. Representatives $w_{i}(i=1,2, \ldots, 11)$ for the $F$-conjugacy classes of $\mathbf{W}$ are given in [19, Table III], in particular:

$$
w_{1}=1, \quad w_{2}=w_{r_{1}}, \quad w_{3}=w_{r_{3}}, \quad w_{4}=w_{r_{2}} w_{r_{3}} w_{r_{2}} .
$$

In this article we will only deal with the maximal torus $T_{1}$. But since we plan to study characters of maximal parabolic subgroups of ${ }^{2} F_{4}\left(q^{2}\right)$ in subsequent work, we give a short description of all conjugacy classes of ${ }^{2} F_{4}\left(q^{2}\right)$ having non-empty intersection with one of the proper parabolic subgroups of ${ }^{2} F_{4}\left(q^{2}\right)$. For this reason we also describe the tori $T_{2}, T_{3}, T_{4}$. The sets $T_{j}:=\mathbf{T}^{\left(F w_{j}^{-1}\right)}$ of fixed points are given by Table 6 . 
Table 6: Some maximal tori of ${ }^{2} F_{4}\left(q^{2}\right)$.

$T_{1}=\left\{h\left(z_{1}, z_{1}^{2 \theta-1}, z_{2}, z_{2}^{2 \theta-1}\right) \mid z_{1}^{q^{2}-1}=z_{2}^{q^{2}-1}=1\right\}$
$T_{2}=\left\{h\left(z^{4 \theta^{3}+2 \theta^{2}+1}, z^{2 \theta^{2}+2 \theta-1}, z^{-2 \theta^{2}+2 \theta+1}, z^{-4 \theta^{3}+2 \theta^{2}+1}\right) \mid z^{q^{4}-1}=1\right\}$
$T_{3}=\left\{h\left(z^{2 \theta^{2}-2 \theta+1}, z^{4 \theta^{3}-6 \theta^{2}+4 \theta-1}, z^{2 \theta^{2}-1}, z^{-4 \theta^{4}+2 \theta^{2}}\right) \mid z^{\left(q^{2}-\sqrt{2} q+1\right)\left(q^{2}-1\right)}=1\right\}$
$T_{4}=\left\{h\left(z^{2 \theta^{2}+2 \theta+1}, z^{4 \theta^{3}+2 \theta^{2}-1}, z^{2 \theta^{2}-1}, z^{-4 \theta^{4}+2 \theta^{2}}\right) \mid z^{\left(q^{2}+\sqrt{2} q+1\right)\left(q^{2}-1\right)}=1\right\}$

A set of representatives for those conjugacy classes of ${ }^{2} F_{4}\left(q^{2}\right)$ having non-empty intersection with one of the proper parabolic subgroups is given in Tables A.1 and A.2 in the Appendix, see [19, Tables II, IV, V]. In this table $\zeta$ is an element of $\mathbb{F}_{q^{2}}$ such that the polynomial $X^{2 \theta-1}+X^{\theta-1}+\zeta$ does not have a root in $\mathbb{F}_{q^{2}}$. Such an element $\zeta$ exists because the map $\mathbb{F}_{q^{2}} \rightarrow \mathbb{F}_{q^{2}}, x \mapsto x^{2 \theta-1}+x^{\theta-1}$ is not injective, hence not surjective. Note that there is a mistake in [19, Table II]: the representative $u_{9}$ is conjugate to $u_{7}$ for certain values of $q$. This is why we choose a different representative for the class $c_{1,9}$.

The unipotent elements $x, x^{\prime}, x^{\prime \prime}$ occuring in Table A.2 depend on the congruence class of $\theta$ modulo 3 and are defined as follows:

$$
\begin{aligned}
x & :=x_{\beta_{1}}(1) x_{\beta_{2}}(1) x_{17}\left(\tau_{0}\right) x_{\gamma_{1}}(1) x_{\gamma_{2}}(1) x_{22}\left(\tau_{0}^{2 \theta}\right) \quad \text { and } \\
x^{\prime} & :=x_{\beta_{1}}(\eta) x_{\beta_{2}}\left(\eta^{l}\right) x_{17}\left(\tau_{1}\right) x_{\gamma_{1}}\left(\eta^{2 \theta}\right) x_{\gamma_{2}}\left(\eta^{2 \theta l}\right) x_{22}\left(\tau_{1}^{2 \theta}\right) \quad \text { and } \\
x^{\prime \prime} & :=x_{\beta_{1}}\left(\eta^{2}\right) x_{\beta_{2}}\left(\eta^{2 l}\right) x_{17}\left(\tau_{2}\right) x_{\gamma_{1}}\left(\eta^{4 \theta}\right) x_{\gamma_{2}}\left(\eta^{4 \theta l}\right) x_{22}\left(\tau_{2}^{2 \theta}\right),
\end{aligned}
$$

where

$$
\begin{cases}\beta_{1}=8, \beta_{2}=10, \gamma_{1}=18, \gamma_{2}=9 & \text { if } \theta \equiv 1 \bmod 3, \\ \beta_{1}=6, \beta_{2}=12, \gamma_{1}=11, \gamma_{2}=16 & \text { if } \theta \equiv-1 \bmod 3,\end{cases}
$$

for details see $[19,(3.3)]$.

\section{The character table of a Borel subgroup $B$}

Let $\mathbf{B}$ be the $F$-stable Borel subgroup $\mathbf{T U}$ of $\mathbf{G}$ where $\mathbf{U}$ is the product of all root subgroups of $\mathbf{G}$ to positive roots, and let $B:=\mathbf{B}^{F}$ be the corresponding Borel subgroup of $\mathbf{G}^{F}$.

Using the relations in Tables $1-4$ and the permutation character $\mathbf{1}_{B}^{G}$ one can determine the conjugacy classes of $B$ and the class fusions from $B$ to $G$. The calculations are similar to those for the Borel subgroup of Steinberg's triality groups ${ }^{3} D_{4}(q)$, see [7, Section 3.8]. We give a short description of the conjugacy classes of $B$. The group $B$ is the semidirect product of $T=\mathbf{T}^{F}$ and the unipotent normal subgroup $U=\mathbf{U}^{F}$. Every $x \in U$ can be written uniquely as

$$
x=\alpha_{1}\left(d_{1}\right) \alpha_{2}\left(d_{2}\right) \alpha_{3}\left(d_{3}\right) \cdots \alpha_{12}\left(d_{12}\right)
$$

with $d_{i} \in \mathbb{F}_{q^{2}}$. The elements of $T$ form a set of representatives for the semisimple conjugacy classes of $B$ and we parametrize these classes according to Table A.3 in the Appendix. A list of all $q^{4}+22 q^{2}+13$ conjugacy classes of $B$ is given in Table A.4 in the Appendix. The parameter sets $I_{1}, I_{2}, \ldots, I_{16}$ and the parameters $t_{a}$ in the 
representatives for the conjugacy classes of type $c_{1,36}$ and $c_{1,37}$ in Table A.4 are defined as follows: For every $a \in \mathbb{F}_{q^{2}}^{\times}$we choose an element $t_{a} \in \mathbb{F}_{q^{2}}-\left\{c^{2 \theta}+a c \mid c \in\right.$ $\mathbb{F}_{q^{2}}$. For group elements $x, y \in G$ we write $x \sim_{G} y$ if $x$ and $y$ are conjugate in $G$. We define:

$$
\begin{aligned}
I_{1} & :=I_{2}:=I_{7}:=\mathbb{F}_{q^{2}}-\{0,1\} \\
I_{3} & :=I_{6}:=\left\{a \in \mathbb{F}_{q^{2}}-\{0,1\} \mid X^{2 \theta}+a X+a \text { has a root in } \mathbb{F}_{q^{2}}\right\} \\
I_{4} & :=I_{5}:=\left\{a \in \mathbb{F}_{q^{2}}-\{0,1\} \mid X^{2 \theta}+a X+a \text { has no root in } \mathbb{F}_{q^{2}}\right\} \\
I_{8} & :=\left\{a \in \mathbb{F}_{q^{2}}^{\times} \mid \alpha_{2}(1) \alpha_{6}(a) \alpha_{8}(1) \sim_{G} \alpha_{5}(1) \alpha_{6}(1)\right\} \\
I_{9} & :=\left\{a \in \mathbb{F}_{q^{2}}^{\times} \mid \alpha_{2}(1) \alpha_{6}(a) \alpha_{8}(1) \sim_{G} \alpha_{5}(1) \alpha_{6}(1) \alpha_{8}(1)\right\} \\
I_{10} & :=\left\{a \in \mathbb{F}_{q^{2}}^{\times} \mid \alpha_{2}(1) \alpha_{6}(a) \alpha_{8}(1) \sim_{G} \alpha_{2}(1) \alpha_{6}(\zeta) \alpha_{8}(1)\right\} \\
I_{11} & :=I_{14}:=\left\{x^{2}+x \mid x \in \mathbb{F}_{q^{2}}-\{0,1\}\right\} \\
I_{12} & :=\left\{a \in \mathbb{F}_{q^{2}}^{\times} \mid \alpha_{1}(1) \alpha_{6}(1) \alpha_{8}(a) \sim_{G} \alpha_{2}(1) \alpha_{4}(1) \alpha_{5}(1)\right\} \\
I_{13} & \left.:=\left\{a \in \mathbb{F}_{q^{2}}^{\times} \mid \alpha_{1}(1) \alpha_{6}(1) \alpha_{8}(a) \sim_{G} \alpha_{2}(1) \alpha_{4}(1) \alpha_{5}(1)\right\} \alpha_{8}(1)\right\}, \\
I_{15} & :=\left\{a \in \mathbb{F}_{q^{2}}^{\times} \mid \alpha_{1}(1) \alpha_{2}(1) \alpha_{6}(1) \alpha_{8}(a) \sim_{G} \alpha_{2}(1) \alpha_{4}(1) \alpha_{5}(1)\right\} \\
I_{16} & :=\left\{a \in \mathbb{F}_{q^{2}}^{\times} \mid \alpha_{1}(1) \alpha_{2}(1) \alpha_{6}(1) \alpha_{8}(a) \sim_{G} \alpha_{2}(1) \alpha_{4}(1) \alpha_{5}(1) \alpha_{8}(1)\right\} .
\end{aligned}
$$

The next lemma will be used to calculate the values of several characters of $B$.

LEMMA 4.1. The following holds:

(a) $\left|\left\{(u, v) \in \mathbb{F}_{q^{2}} \times \mathbb{F}_{q^{2}}^{\times} \mid v^{2 \theta}+u v+u^{2 \theta+2}=0\right\}\right|=0$,

(b) $\left|\left\{(u, v) \in \mathbb{F}_{q^{2}} \times \mathbb{F}_{q^{2}}^{\times} \mid v^{2 \theta}+u v+u^{2 \theta+2}+u=0\right\}\right|=q^{2}-1$.

Proof. (a) Using the substitution $y=u v^{1-2 \theta}$ we get

$$
\begin{gathered}
\left|\left\{(u, v) \in \mathbb{F}_{q^{2}} \times \mathbb{F}_{q^{2}}^{\times} \mid v^{2 \theta}+u v+u^{2 \theta+2}=0\right\}\right|= \\
\left|\left\{(u, v) \in \mathbb{F}_{q^{2}} \times \mathbb{F}_{q^{2}}^{\times} \mid 1+u v^{1-2 \theta}+u^{2 \theta+2} v^{-2 \theta}=0\right\}\right|= \\
\left|\left\{(y, v) \in \mathbb{F}_{q^{2}} \times \mathbb{F}_{q^{2}}^{\times} \mid 1+y+y^{2 \theta+2}=0\right\}\right| .
\end{gathered}
$$

Suppose there is $y \in \mathbb{F}_{q^{2}}$ with $y^{2 \theta+2}+y+1=0$. Then there is $x \in \mathbb{F}_{q^{2}}^{\times}$with $y=x^{2(\theta-1)}$. Plugging in, we get $x^{-2}+x^{2 \theta-2}+1=0$ and hence $1+x^{2 \theta}+x^{2}=0$. Raising this equation to the $2 \theta$ th power and adding up we get $x^{4 \theta}=x^{2 \theta}$ and thus $x=0$ or $x=1$, a contradiction.

(b) Using the substitution $y=u v^{1-2 \theta}$ we have

$$
\begin{gathered}
\left|\left\{(u, v) \in \mathbb{F}_{q^{2}} \times \mathbb{F}_{q^{2}}^{\times} \mid v^{2 \theta}+u v+u^{2 \theta+2}+u=0\right\}\right|= \\
\left|\left\{(u, v) \in \mathbb{F}_{q^{2}}^{\times} \times \mathbb{F}_{q^{2}}^{\times} \mid 1+u v^{1-2 \theta}+u^{2 \theta+2} v^{-2 \theta}+u v^{-2 \theta}=0\right\}\right|= \\
\left|\left\{(y, v) \in \mathbb{F}_{q^{2}}^{\times} \times \mathbb{F}_{q^{2}}^{\times} \mid 1+y+y^{2 \theta+2}+v^{-1} y=0\right\}\right|= \\
\left|\left\{(y, v) \in \mathbb{F}_{q^{2}}^{\times} \times \mathbb{F}_{q^{2}}^{\times} \mid y^{-1}+1+y^{2 \theta+1}+v^{-1}=0\right\}\right|= \\
\left|\left\{y \in \mathbb{F}_{q^{2}}^{\times} \mid y^{-1}+1+y^{2 \theta+1} \neq 0\right\}\right|= \\
q^{2}-1-\left|\left\{y \in \mathbb{F}_{q^{2}}^{\times} \mid y^{2 \theta+2}+y+1=0\right\}\right|=q^{2}-1
\end{gathered}
$$

since the set occurring in the last equation is empty as we have seen in part (a). 
Now, we start to construct the irreducible characters of $B$. For abbreviation, set $\pi:=\tilde{\zeta}_{2}$ such that $\pi$ is a generator of $\mathbb{F}_{q^{2}}^{\times}$. Fix a nontrivial linear character $\phi: \mathbb{F}_{q^{2}} \rightarrow \mathbb{C}^{\times}$of the additive group of $\mathbb{F}_{q^{2}}$ such that $\left\{u^{2}+u \mid u \in \mathbb{F}_{q^{2}}\right\} \subseteq \operatorname{ker}(\phi)$ and $\phi(1)=-1$ (this is possible since $1 \notin\left\{u^{2}+u \mid u \in \mathbb{F}_{q^{2}}\right\}$ ). By Problem (2.1) in [12] we have:

$$
\begin{aligned}
& \sum_{t \in \mathbb{F}_{q^{2}}} \phi(t)=0 \quad \text { and } \\
& \sum_{t \in \mathbb{F}_{q^{2}}^{\times}} \phi(t)=-1 .
\end{aligned}
$$

Furthermore:

$$
\phi\left(u^{2}\right)=\phi(u) \text { for all } u \in \mathbb{F}_{q^{2}}
$$

since $\left\{u^{2}+u \mid u \in \mathbb{F}_{q^{2}}\right\} \subseteq \operatorname{ker}(\phi)$. For $i=1,2, \ldots, 12$, let $U_{i}:=\left\{\alpha_{i}(t) \mid t \in \mathbb{F}_{q^{2}}\right\}$ and $U^{i}:=U_{i} U_{i+1} \cdots U_{12}$. Note that each $U^{i}$ is a normal subgroup of $B$. Each element of $B$ can be written uniquely as

$$
h\left(z_{1}, z_{2}\right) \alpha_{1}\left(d_{1}\right) \alpha_{2}\left(d_{2}\right) \alpha_{3}\left(d_{3}\right) \cdots \alpha_{12}\left(d_{12}\right)
$$

with $z_{1}, z_{2} \in \mathbb{F}_{q^{2}}^{\times}, d_{1}, d_{2}, \ldots, d_{12} \in \mathbb{F}_{q^{2}} \subseteq \mathbb{F}$. The irreducible characters of $B$ can be constructed as follows:

For $k, l=0, \ldots, q^{2}-2$, let ${ }_{B} \chi_{1}(k, l)$ be the linear character of $B$ defined by

$$
h\left(\pi^{i}, \pi^{j}\right) \alpha_{1}\left(d_{1}\right) \alpha_{2}\left(d_{2}\right) \alpha_{3}\left(d_{3}\right) \cdots \alpha_{12}\left(d_{12}\right) \mapsto \zeta_{2}^{i k+j l} .
$$

For $k=0, \ldots, q^{2}-2$, define a linear character of $C_{T}\left(\alpha_{1}(1)\right) U$ by

$$
h_{2}(i) \alpha_{1}\left(d_{1}\right) \alpha_{2}\left(d_{2}\right) \alpha_{3}\left(d_{3}\right) \cdots \alpha_{12}\left(d_{12}\right) \mapsto \zeta_{2}^{i k} \phi\left(d_{1}\right) .
$$

Inducing this character to $B$, we obtain $B \chi_{2}(k)$.

For $k=0, \ldots, q^{2}-2$, define a linear character of $C_{T}\left(\alpha_{3}(1)\right) U$ by

$$
h_{3}(i) \alpha_{1}\left(d_{1}\right) \alpha_{2}\left(d_{2}\right) \alpha_{3}\left(d_{3}\right) \cdots \alpha_{12}\left(d_{12}\right) \mapsto \zeta_{2}^{i k} \phi\left(d_{3}\right) .
$$

Inducing this character to $B$, we obtain ${ }_{B} \chi_{5}(k)$. Let ${ }_{B} \chi_{8}$ be the character of $B$ induced from the following linear character of $U$ :

$$
\alpha_{1}\left(d_{1}\right) \alpha_{2}\left(d_{2}\right) \alpha_{3}\left(d_{3}\right) \cdots \alpha_{12}\left(d_{12}\right) \mapsto \phi\left(d_{1}+d_{3}\right) .
$$

Let ${ }_{B} \chi_{9}(k), k=0, \ldots, q^{2}-2$, be the character of $B$ induced from the following linear character of $C_{T}\left(\alpha_{4}(1)\right) U^{2}$ :

$$
h_{4}(i) \alpha_{2}\left(d_{2}\right) \alpha_{3}\left(d_{3}\right) \alpha_{4}\left(d_{4}\right) \cdots \alpha_{12}\left(d_{12}\right) \mapsto \zeta_{2}^{i k} \phi\left(d_{4}\right) .
$$

Let $B \chi_{10}$ be the character of $B$ induced from the following linear character of $U^{2}$ :

$$
\alpha_{2}\left(d_{2}\right) \alpha_{3}\left(d_{3}\right) \alpha_{4}\left(d_{4}\right) \cdots \alpha_{12}\left(d_{12}\right) \mapsto \phi\left(d_{2}+d_{4}\right) .
$$

Let ${ }_{B} \chi_{11}(k), k=0, \ldots, q^{2}-2$, be the character of $B$ induced from the following linear character of $C_{T}\left(\alpha_{5}(1)\right) U^{3}$ :

$$
h_{5}(i) \alpha_{3}\left(d_{3}\right) \alpha_{4}\left(d_{4}\right) \alpha_{5}\left(d_{5}\right) \cdots \alpha_{12}\left(d_{12}\right) \mapsto \zeta_{2}^{i k} \phi\left(d_{5}\right) .
$$

Let ${ }_{B} \chi_{12}(k), k=0, \ldots, q^{2}-2$, be the character of $B$ induced from the following linear character of $C_{T}\left(\alpha_{6}(1)\right) U^{3}$ :

$$
h_{6}(i) \alpha_{3}\left(d_{3}\right) \alpha_{4}\left(d_{4}\right) \alpha_{5}\left(d_{5}\right) \cdots \alpha_{12}\left(d_{12}\right) \mapsto \zeta_{2}^{i k} \phi\left(d_{6}\right) .
$$


Let ${ }_{B} \chi_{23}(k), k=0, \ldots, q^{2}-2$, be the character of $B$ induced from the following linear character of $C_{T}\left(\alpha_{7}(1)\right) U^{3}$ :

$$
h_{7}(i) \alpha_{3}\left(d_{3}\right) \alpha_{4}\left(d_{4}\right) \alpha_{5}\left(d_{5}\right) \cdots \alpha_{12}\left(d_{12}\right) \mapsto \zeta_{2}^{i k} \phi\left(d_{7}\right) .
$$

Let ${ }_{B} \chi_{24}$ be the character of $B$ induced from the following linear character of $U^{3}$ :

$$
\alpha_{3}\left(d_{3}\right) \alpha_{4}\left(d_{4}\right) \alpha_{5}\left(d_{5}\right) \cdots \alpha_{12}\left(d_{12}\right) \mapsto \phi\left(d_{3}+d_{7}\right) .
$$

Let ${ }_{B} \chi_{38}$ be the character of $B$ induced from the following linear character of $U_{2} U^{5}$ :

$$
\alpha_{2}\left(d_{2}\right) \alpha_{5}\left(d_{5}\right) \alpha_{6}\left(d_{6}\right) \alpha_{7}\left(d_{7}\right) \alpha_{8}\left(d_{8}\right) \cdots \alpha_{12}\left(d_{12}\right) \mapsto \phi\left(d_{7}+d_{8}\right) .
$$

Let ${ }_{B} \chi_{39}(k), k=0, \ldots, q^{2}-2$, be the character of $B$ induced from the following linear character of $C_{T}\left(\alpha_{9}(1)\right) U_{2} U^{5}$ :

$$
h_{8}(i) \alpha_{2}\left(d_{2}\right) \alpha_{5}\left(d_{5}\right) \alpha_{6}\left(d_{6}\right) \cdots \alpha_{9}\left(d_{9}\right) \cdots \alpha_{12}\left(d_{12}\right) \mapsto \zeta_{2}^{i k} \phi\left(d_{9}\right) .
$$

Let ${ }_{B} \chi_{40}$ be the character of $B$ induced from the following linear character of $U_{2} U^{5}$ :

$$
\alpha_{2}\left(d_{2}\right) \alpha_{5}\left(d_{5}\right) \alpha_{6}\left(d_{6}\right) \alpha_{7}\left(d_{7}\right) \alpha_{8}\left(d_{8}\right) \cdots \alpha_{12}\left(d_{12}\right) \mapsto \phi\left(d_{2}+d_{9}\right) .
$$

Let ${ }_{B} \chi_{42}(k), k=0, \ldots, q^{2}-2$, be the character of $B$ induced from the following linear character of $C_{T}\left(\alpha_{10}(1)\right) U_{3} U_{4} U_{6} U^{8}$ :

$$
h_{9}(i) \alpha_{3}\left(d_{3}\right) \alpha_{4}\left(d_{4}\right) \alpha_{6}\left(d_{6}\right) \alpha_{8}\left(d_{8}\right) \alpha_{9}\left(d_{9}\right) \alpha_{10}\left(d_{10}\right) \cdots \alpha_{12}\left(d_{12}\right) \mapsto \zeta_{2}^{i k} \phi\left(d_{10}\right)
$$

Let $B \chi_{43}$ be the character of $B$ induced from the following linear character of $U_{3} U_{4} U_{6} U^{8}$ :

$$
\alpha_{3}\left(d_{3}\right) \alpha_{4}\left(d_{4}\right) \alpha_{6}\left(d_{6}\right) \alpha_{8}\left(d_{8}\right) \alpha_{9}\left(d_{9}\right) \alpha_{10}\left(d_{10}\right) \cdots \alpha_{12}\left(d_{12}\right) \mapsto \phi\left(d_{3}+d_{10}\right) .
$$

It is not difficult to compute the values of the above characters using (1), (2), (3) and Lemma 4.1. We demonstrate how to determine the values of ${ }_{B} \chi_{23}(k)$, $k=0, \ldots, q^{2}-2$, on the conjugacy classes $c_{1,32}, c_{1,33}, c_{1,34}(a), c_{1,35}(a), c_{1,36}(a)$, $c_{1,37}(a)$ of $B$ : The set

$$
\left\{h_{i^{\prime}, u, v}:=h\left(\pi^{i^{\prime}}, 1\right) \alpha_{1}(u) \alpha_{2}(v) \mid i^{\prime}=1, \ldots, q^{2}-1, u, v \in \mathbb{F}_{q^{2}}\right\}
$$

is a set of representatives for the right cosets of $C_{T}\left(\alpha_{7}(1)\right) U^{3}$ in $B$ and for all $a \in \mathbb{F}_{q^{2}}^{\times}$and $d_{8} \in \mathbb{F}_{q^{2}}$ the element ${ }^{h_{i^{\prime}, u, v}}\left(\alpha_{3}(1) \alpha_{5}(a) \alpha_{6}(1) \alpha_{8}\left(d_{8}\right)\right)$ is equal to

$$
\alpha_{3}(\ldots) \cdots \alpha_{7}\left(\left(v^{2 \theta}+u v+u^{2 \theta+2}+u\right) \pi^{(2 \theta-1) i^{\prime}}\right) \cdots \alpha_{12}(\ldots) .
$$

Hence by the definition of induced characters (see [12, p. 62]), the value of ${ }_{B} \chi_{23}(k)$ on a conjugacy class of $B$ with representative $\alpha_{3}(1) \alpha_{5}(a) \alpha_{6}(1) \alpha_{8}\left(d_{8}\right)$ is

$$
\begin{gathered}
\sum_{i^{\prime}=1}^{q^{2}-1} \sum_{u, v \in \mathbb{F}_{q^{2}}} \phi\left(\left(v^{2 \theta}+u v+u^{2 \theta+2}+u\right) \pi^{(2 \theta-1) i^{\prime}}\right)= \\
\sum_{i^{\prime}=1}^{q^{2}-1} \sum_{u, v \in \mathbb{F}_{q^{2}}} \phi\left(\left(v^{2 \theta}+u v+u^{2 \theta+2}+u\right) \pi^{i^{\prime}}\right) .
\end{gathered}
$$

By Lemma $4.1(\mathrm{~b})$, the number of pairs $(u, v) \in \mathbb{F}_{q^{2}}$ with $v^{2 \theta}+u v+u^{2 \theta+2}+u=0$ 
is $q^{2}-1+2=q^{2}+1$. So, by (2) we have

$$
\begin{gathered}
\sum_{i^{\prime}=1}^{q^{2}-1} \sum_{u, v \in \mathbb{F}_{q^{2}}} \phi\left(\left(v^{2 \theta}+u v+u^{2 \theta+2}+u\right) \pi^{i^{\prime}}\right)= \\
\left(q^{2}+1\right) \cdot\left(q^{2}-1\right)+\left(q^{4}-\left(q^{2}+1\right)\right) \cdot(-1)=q^{2} .
\end{gathered}
$$

This gives the value of ${ }_{B} \chi_{23}(k)$ on the classes $c_{1,32}, c_{1,33}, c_{1,34}(a), \ldots, c_{1,37}(a)$.

Since $\left({ }_{B} \chi_{j},{ }_{B} \chi_{j}\right)_{B}=1$ for $j=8,10,24,38,40,43$ and $\left({ }_{B} \chi_{j}(k),{ }_{B} \chi_{j}(k)\right)_{B}=1$ for $j=2,5,9,11,12,23,39,42$ and $k$ as in Table A.5 in the Appendix the above characters are irreducible.

Next, we construct the remaining irreducible characters $\chi$ of $B$ with $U^{3} \subseteq \operatorname{ker}(\chi)$. Equivalently, we complete the character table of the factor group $B / U^{3} \cong T U_{1} U_{2}$. The group $L_{b}:=\left\langle T U_{1} U_{2}, n_{b}\right\rangle=C_{T}\left(\alpha_{1}(1) \alpha_{2}(1)\right) \times\left\langle U_{1}, n_{b}\right\rangle \cong \mathbb{Z}_{q^{2}-1} \times \operatorname{Sz}\left(q^{2}\right)$ is a Levi subgroup of the parabolic subgroup $P_{b}$ of ${ }^{2} F_{4}\left(q^{2}\right)$ and $T U_{1} U_{2}$ is a Borel subgroup of $L_{b}$. The character table of $\mathrm{Sz}\left(q^{2}\right)$ is contained in the CHEVIE library so that we can write down explicitly the irreducible characters of $L_{b}$. In particular, we see that $L_{b}$ has two families of $q^{2}-1$ irreducible characters of degree $\frac{q}{\sqrt{2}}\left(q^{2}-1\right)$. Restricting these characters to $T U_{1} U_{2}$ we obtain characters $\psi(k)$ and $\psi^{\prime}(k), k=$ $0,1, \ldots, q^{2}-2$ of $T U_{1} U_{2}$ such that, for every $k$, the characters $\psi(k)$ and $\psi^{\prime}(k)$ are complex-conjugate to each other. Inflating the $\psi(k)$ and $\psi^{\prime}(k)$ to $B$ gives us the characters ${ }_{B} \chi_{3}(k),{ }_{B} \chi_{4}(k)$ for $k=0,1,2 \ldots, q^{2}-2$. Computing scalar products with CHEVIE we see that these characters are irreducible. This completes the determination of the irreducible characters $\chi$ of $B$ with $U^{3} \subseteq \operatorname{ker}(\chi)$.

Let ${ }_{B} \chi_{6}:={ }_{B} \chi_{3}(0) \cdot{ }_{B} \chi_{5}(0)$ and ${ }_{B} \chi_{7}:={ }_{B} \chi_{4}(0) \cdot{ }_{B} \chi_{5}(0)$.

For $k=0,1$, we define the two linear characters

$$
\varphi_{k}: \alpha_{2}(1)^{i} \alpha_{3}\left(d_{3}\right) \alpha_{4}\left(d_{4}\right) \cdots \alpha_{12}\left(d_{12}\right) \mapsto(-1)^{i k} \phi\left(d_{5}+d_{6}\right)
$$

of $\left\langle\alpha_{2}(1)\right\rangle U^{3}$ and let ${ }_{B} \chi_{13}:=\varphi_{0}^{B}$ and ${ }_{B} \chi_{14}:=\varphi_{1}^{B}$.

Next, we construct ${ }_{B} \chi_{15}, B \chi_{16}, \ldots, B \chi_{22}$. We fix $\varepsilon_{4}=e^{2 \pi i / 4} \in \mathbb{C}$. For $k=0,1$ and $l=0,1,2,3$ we define a linear character of $\left\langle\alpha_{1}(1)\right\rangle U^{3}$ by

$$
\varphi_{k l}: \alpha_{1}(1)^{i} \alpha_{3}\left(d_{3}\right) \alpha_{4}\left(d_{4}\right) \alpha_{5}\left(d_{5}\right) \alpha_{6}\left(d_{6}\right) \cdots \alpha_{12}\left(d_{12}\right) \mapsto \varepsilon_{4}^{i l} \phi\left(k \cdot d_{3}+d_{4}+d_{5}+d_{6}\right) .
$$

Using (1), (2), (3) we can compute the values of $\varphi_{k l}^{B}$ and can then verify that $\left(\varphi_{k l}^{B}, \varphi_{k l}^{B}\right)_{B}=1$ for all $k, l$. So we get eight irreducible characters ${ }_{B} \chi_{15}, \ldots, \chi_{22}$.

Number the elements of $\mathbb{F}_{q^{2}}$ in some way, say $\mathbb{F}_{q^{2}}=\left\{x_{1}, x_{2}, x_{3}, \ldots, x_{q^{2}}\right\}$ with $x_{1}=0$. Let ${ }_{B} \chi_{25}(k), k=1, \ldots, q^{2}$, be the character of $B$ induced from the following linear character of $U^{3}: \alpha_{3}\left(d_{3}\right) \alpha_{4}\left(d_{4}\right) \alpha_{5}\left(d_{5}\right) \cdots \alpha_{12}\left(d_{12}\right) \mapsto \phi\left(x_{k} \cdot d_{3}+d_{5}+d_{7}\right)$. It is not difficult to compute the values of $\sum_{k=1}^{q^{2}} \chi_{25}(k)$. Using CHEVIE it is then easy to verify $\left(\sum_{k=1}^{q^{2}} B \chi_{25}(k), \sum_{k=1}^{q^{2}} B \chi_{25}(k)\right)_{B}=q^{2}$. Hence, the ${ }_{B} \chi_{25}(k)$ are $q^{2}$ different irreducible characters.

Next, we construct the characters ${ }_{B} \chi_{26}(k),{ }_{B} \chi_{27}(k),{ }_{B} \chi_{44}(k),{ }_{B} \chi_{45}(k),{ }_{B} \chi_{51}(k)$, ${ }_{B} \chi_{52}(k)$. The subgroup $H:=T U_{1} U_{2} U_{4} U_{5} U_{6} U_{7} \cdots U_{12}$ of $B$ is the semidirect product $H=T U_{4} U_{8} \ltimes U_{1} U_{2} U_{5} U_{6} U_{7} U_{9} U_{10} U_{11} U_{12}$ where $T U_{4} U_{8}$ is isomorphic to $T U_{1} U_{2}$. So by the construction of ${ }_{B} \chi_{3}(k),{ }_{B} \chi_{4}(k), T U_{4} U_{8}$ has two families of $q^{2}-1$ irreducible characters of degree $q\left(q^{2}-1\right) / \sqrt{2}$. By abuse of notation we denote these characters in the same way as for $T U_{1} U_{2}$ by $\psi(k)$ and $\psi^{\prime}(k), k=0,1, \ldots, q^{2}-2$, respectively. For every $k$ the characters $\psi(k)$ and $\psi^{\prime}(k)$ are complex-conjugate to each 
other and we have $\psi(k)(1)=q\left(q^{2}-1\right) / \sqrt{2}, \psi(k)\left(\alpha_{8}(1)\right)=-q / \sqrt{2}, \psi(k)\left(\alpha_{4}(1)\right)=$ $i q \sqrt{2}, \psi(k)\left(\alpha_{4}(1) \alpha_{8}(1)\right)=-i q / \sqrt{2}$ and $\psi(k)\left(h_{4}(i) u\right)=\zeta_{2}^{i k} \psi(k)(u)$ for all $u \in U_{4} U_{8}$. Inflating $\psi(k)$ and $\psi^{\prime}(k)$ to $H$ and inducing up to $B$ we get the characters $B \chi_{26}(k)$, ${ }_{B} \chi_{27}(k)$. For every $k$, by construction, the characters ${ }_{B} \chi_{26}(k),{ }_{B} \chi_{27}(k)$ are complexconjugate to each other.

Similarly, we construct the characters ${ }_{B} \chi_{44}(k),{ }_{B} \chi_{45}(k), k=0,1, \ldots, q^{2}-2$, from the subgroup $T U_{3} U_{4} U_{5} U_{6} U_{8} U_{9} U_{10} U_{11} U_{12}=T U_{6} U_{11} \ltimes U_{3} U_{4} U_{5} U_{8} U_{9} U_{10} U_{12}$ and the characters ${ }_{B} \chi_{51}(k),{ }_{B} \chi_{52}(k), k=0,1, \ldots, q^{2}-2$, from the subgroup $T U_{1} U_{2} U_{5} U_{6} U_{7} U_{10} U_{11} U_{12}=T U_{5} U_{12} \ltimes U_{1} U_{2} U_{6} U_{7} U_{10} U_{11}$. For every $k$, the characters ${ }_{B} \chi_{44}(k),{ }_{B} \chi_{45}(k)$ are complex-conjugate to each other and the same holds for ${ }_{B} \chi_{51}(k),{ }_{B} \chi_{52}(k)$.

The determination of the values of the characters ${ }_{B} \chi_{26}(k),{ }_{B} \chi_{27}(k),{ }_{B} \chi_{44}(k)$, ${ }_{B} \chi_{45}(k),{ }_{B} \chi_{51}(k)$ and ${ }_{B} \chi_{52}(k)$ is a non-trivial task. First, we only deal with the characters ${ }_{B} \chi_{26}(k)+{ }_{B} \chi_{27}(k),{ }_{B} \chi_{44}(k)+{ }_{B} \chi_{45}(k)$ and ${ }_{B} \chi_{51}(k)+{ }_{B} \chi_{52}(k)$ so that we can ignore the imaginary parts of the character values.

Using only the definition of induced characters and Lemma 4.1, it is not difficult to determine the values of ${ }_{B} \chi_{26}(k)+{ }_{B} \chi_{27}(k),{ }_{B} \chi_{44}(k)+{ }_{B} \chi_{45}(k),{ }_{B} \chi_{51}(k)+{ }_{B} \chi_{52}(k)$ except for the values of ${ }_{B} \chi_{26}(k)+{ }_{B} \chi_{27}(k)$ on the conjugacy classes $c_{1,46}(a), c_{1,47}(a)$, $c_{1,48}(a)$. For $a \in I_{8} \cup I_{9} \cup I_{10}$ we get

$$
\left({ }_{B} \chi_{26}(k)+{ }_{B} \chi_{27}(k)\right)\left(\alpha_{2}(1) \alpha_{6}(a) \alpha_{8}(1)\right)=\left(M_{a}-1\right) \sqrt{2} q^{3},
$$

where $M_{a}:=\left|\left\{x \in \mathbb{F}_{q^{2}} \mid x^{2 \theta+1}+a x^{2 \theta}+1=0\right\}\right|$. To determine the missing values of the character ${ }_{B} \chi_{26}(k)+{ }_{B} \chi_{27}(k)$ we use the two irreducible unipotent characters ${ }_{G} \chi_{2}$ and ${ }_{G} \chi_{3}$ of degree $q\left(q^{2}-1\right)\left(q^{2}+1\right)^{2}\left(q^{4}-q^{2}+1\right) / \sqrt{2}$ of $G={ }^{2} F_{4}\left(q^{2}\right)$ (see the remarks in Section 3). In particular, $G_{G} \chi_{2}$ and ${ }_{G} \chi_{3}$ are complex-conjugate to each other.

Using CHEVIE we can calculate $\left({ }_{B} \chi_{44}(k)+{ }_{B} \chi_{45}(k),{ }_{B} \chi_{44}(k)+{ }_{B} \chi_{45}(k)\right)_{B}=$ 2 and $\left({ }_{B} \chi_{51}(k)+{ }_{B} \chi_{52}(k),{ }_{B} \chi_{51}(k)+{ }_{B} \chi_{52}(k)\right)_{B}=2$. The obvious bounds $0 \leqslant$ $M_{a} \leqslant 2 \theta+1$ imply $\left({ }_{B} \chi_{26}(k)+{ }_{B} \chi_{27}(k),{ }_{B} \chi_{26}(k)+{ }_{B} \chi_{27}(k)\right)_{B}=2$ for all $k$. So the characters ${ }_{B} \chi_{26}(k),{ }_{B} \chi_{27}(k),{ }_{B} \chi_{44}(k),{ }_{B} \chi_{45}(k),{ }_{B} \chi_{51}(k),{ }_{B} \chi_{52}(k)$ are different irreducible characters for all $k$. Furthermore, we can compute the scalar products $\left(\left(G_{G} \chi_{2}\right)_{B},\left(G_{G} \chi_{2}\right)_{B}\right)_{B}=\left(\left(G_{G} \chi_{3}\right)_{B},\left(G_{G} \chi_{3}\right)_{B}\right)_{B}=4$ and $\left(\left(_{G} \chi_{2}\right)_{B},\left(G_{G} \chi_{3}\right)_{B}\right)_{B}=0$ and

$$
\begin{aligned}
\left({ }_{B} \chi_{3}(0)+{ }_{B} \chi_{4}(0),\left({ }_{G} \chi_{2}\right)_{B}+\left({ }_{G} \chi_{3}\right)_{B}\right)_{B} & =2, \\
\left({ }_{B} \chi_{44}(0)+{ }_{B} \chi_{45}(0),\left({ }_{G} \chi_{2}\right)_{B}+\left({ }_{G} \chi_{3}\right)_{B}\right)_{B} & =2, \\
\left({ }_{B} \chi_{51}(0)+{ }_{B} \chi_{52}(0),\left({ }_{G} \chi_{2}\right)_{B}+\left({ }_{G} \chi_{3}\right)_{B}\right)_{B} & =2 .
\end{aligned}
$$

So, $\left(G_{G} \chi_{2}\right)_{B}$ is a sum of four different irreducible characters of $B$ and the same holds for $\left(G \chi_{3}\right)_{B}$. Furthermore, $\left(G_{G} \chi_{2}\right)_{B},\left({ }_{G} \chi_{3}\right)_{B}$ have no constituent in common. Thus, $\left(G_{G} \chi_{2}\right)_{B}+\left(G_{G} \chi_{3}\right)_{B}$ is a real-valued character and is a sum of eight different irreducible characters. Furthermore, ${ }_{B} \chi_{3}(0),{ }_{B} \chi_{4}(0),{ }_{B} \chi_{44}(0),{ }_{B} \chi_{45}(0),{ }_{B} \chi_{51}(0),{ }_{B} \chi_{52}(0)$ are constituents of $\left(G_{G} \chi_{2}\right)_{B}+\left(G_{G} \chi_{3}\right)_{B}$, each with multiplicity one. Again using the bounds $0 \leqslant M_{a} \leqslant 2 \theta+1$, we get $\left({ }_{B} \chi_{26}(0)+{ }_{B} \chi_{27}(0),\left({ }_{G} \chi_{2}\right)_{B}+\left({ }_{G} \chi_{3}\right)_{B}\right)_{B}>0$ and therefore

$$
\left(G_{G} \chi_{2}\right)_{B}+\left({ }_{G} \chi_{3}\right)_{B}=\sum_{i \in\{3,4,26,27,44,45,51,52\}} B \chi_{i}(0)
$$

From (4) we can compute the missing values of ${ }_{B} \chi_{26}(k)+{ }_{B} \chi_{27}(k)$ (note that the 
missing character values are values on unipotent elements and therefore do not depend on $k$ ). As a consequence we get:

$$
M_{a}=\left|\left\{x \in \mathbb{F}_{q^{2}} \mid x^{2 \theta+1}+a x^{2 \theta}+1=0\right\}\right|= \begin{cases}3 & \text { if } a \in I_{8}, \\ 1 & \text { if } a \in I_{9}, \\ 0 & \text { if } a \in I_{10}\end{cases}
$$

The values of ${ }_{B} \chi_{44}(k),{ }_{B} \chi_{45}(k),{ }_{B} \chi_{51}(k)$ and ${ }_{B} \chi_{52}(k)$ can then be obtained using only the definition of induced characters and (4). Finally, we get the missing values of ${ }_{B} \chi_{26}(k)$ and ${ }_{B} \chi_{27}(k)$ from

$$
\begin{aligned}
& \left({ }_{G} \chi_{2}\right)_{B}={ }_{B} \chi_{3}(0)+{ }_{B} \chi_{26}(0)+{ }_{B} \chi_{44}(0)+{ }_{B} \chi_{51}(0) \text { and } \\
& \left({ }_{G} \chi_{3}\right)_{B}={ }_{B} \chi_{4}(0)+{ }_{B} \chi_{27}(0)+{ }_{B} \chi_{45}(0)+{ }_{B} \chi_{52}(0) .
\end{aligned}
$$

Let ${ }_{B} \chi_{28}:={ }_{B} \chi_{2}(0) \cdot{ }_{B} \chi_{26}(0),{ }_{B} \chi_{29}:={ }_{B} \chi_{2}(0) \cdot{ }_{B} \chi_{27}(0)$. Furthermore, we define ${ }_{B} \chi_{30}:={ }_{B} \chi_{3}(0) \cdot{ }_{B} \chi_{26}(0),{ }_{B} \chi_{31}:={ }_{B} \chi_{4}(0) \cdot{ }_{B} \chi_{26}(0),{ }_{B} \chi_{32}:={ }_{B} \chi_{3}(0) \cdot{ }_{B} \chi_{27}(0)$ and ${ }_{B} \chi_{33}:={ }_{B} \chi_{4}(0) \cdot{ }_{B} \chi_{27}(0)$.

Number the elements of $\mathbb{F}_{q^{2}}$ in some way, say $\mathbb{F}_{q^{2}}=\left\{x_{1}, x_{2}, x_{3}, \ldots, x_{q^{2}}\right\}$ with $x_{1}=0$. Let ${ }_{B} \chi_{41}(k), k=1, \ldots, q^{2}$ be the character of $B$ induced from the following linear character of $U_{2} U^{5}$ :

$$
\alpha_{2}\left(d_{2}\right) \alpha_{5}\left(d_{5}\right) \alpha_{6}\left(d_{6}\right) \alpha_{7}\left(d_{7}\right) \alpha_{8}\left(d_{8}\right) \alpha_{9}\left(d_{9}\right) \cdots \alpha_{12}\left(d_{12}\right) \mapsto \phi\left(x_{k} \cdot d_{2}+d_{7}+d_{9}\right) .
$$

It is not difficult to compute the values of $\sum_{k=1}^{q^{2}} \chi_{41}(k)$. Using CHEVIE it is then easy to verify $\left(\sum_{k=1}^{q^{2}} B \chi_{41}(k), \sum_{k=1}^{q^{2}} B \chi_{41}(k)\right)_{B}=q^{2}$. Hence, the ${ }_{B} \chi_{41}(k)$ are $q^{2}$ different irreducible characters.

$$
\text { Let }{ }_{B} \chi_{46}:={ }_{B} \chi_{5}(0) \cdot{ }_{B} \chi_{44}(0) \text { and }{ }_{B} \chi_{47}:={ }_{B} \chi_{5}(0) \cdot{ }_{B} \chi_{45}(0) \text {. }
$$

Next, we construct the characters ${ }_{B} \chi_{48}(k),{ }_{B} \chi_{49}(k)$. The group $H:=T U_{6} U_{11}$ is isomorphic to $T U_{1} U_{2}$ in a natural way. So by Clifford theory [12, Theorems (6.11) and (6.28), Corollary (6.17)] the group $U_{6} U_{11}$ has two families of $q^{2}-1$ irreducible characters of degree $q / \sqrt{2}$ corresponding to two different orbits under the action of $T$ on $\operatorname{Irr}\left(U_{6} U_{11}\right)$. Choose $\psi \in \operatorname{Irr}\left(U_{6} U_{11}\right)$ in the one family and $\psi^{\prime} \in \operatorname{Irr}\left(U_{6} U_{11}\right)$ in the other family.

Number the elements of $\mathbb{F}_{q^{2}}$ in some way, say $\mathbb{F}_{q^{2}}=\left\{x_{1}, x_{2}, x_{3}, \ldots, x_{q^{2}}\right\}$ with $x_{1}=0$. For $k=1, \ldots, q^{2}$, let ${ }_{B} \chi_{48}(k)$ be the character of $B$ induced from the following character of $U_{3} U_{4} U_{5} U_{6} U^{8}$ :

$$
\alpha_{3}\left(d_{3}\right) \alpha_{4}\left(d_{4}\right) \alpha_{5}\left(d_{5}\right) \cdots \alpha_{12}\left(d_{12}\right) \mapsto \phi\left(x_{k} \cdot d_{3}\right) \psi\left(\alpha_{6}\left(d_{6}\right) \alpha_{11}\left(d_{11}\right)\right)
$$

and let ${ }_{B} \chi_{49}(k)$ be the character of $B$ induced from the following character of $U_{3} U_{4} U_{5} U_{6} U^{8}$ :

$$
\alpha_{3}\left(d_{3}\right) \alpha_{4}\left(d_{4}\right) \alpha_{5}\left(d_{5}\right) \cdots \alpha_{12}\left(d_{12}\right) \mapsto \phi\left(x_{k} \cdot d_{3}\right) \psi^{\prime}\left(\alpha_{6}\left(d_{6}\right) \alpha_{11}\left(d_{11}\right)\right) .
$$

Number the elements of $\mathbb{F}_{q^{2}}$ in some way, say $\mathbb{F}_{q^{2}}=\left\{x_{1}, x_{2}, x_{3}, \ldots, x_{q^{2}}\right\}$ with $x_{1}=0$. Let ${ }_{B} \chi_{50}(k), k=1, \ldots, q^{2}$ be the character of $B$ induced from the following linear character of $U_{3} U_{4} U_{5} U^{8}$ :

$\alpha_{3}\left(d_{3}\right) \alpha_{4}\left(d_{4}\right) \alpha_{5}\left(d_{5}\right) \alpha_{8}\left(d_{8}\right) \alpha_{9}\left(d_{9}\right) \alpha_{10}\left(d_{10}\right) \alpha_{11}\left(d_{11}\right) \alpha_{12}\left(d_{12}\right) \mapsto \phi\left(x_{k} \cdot d_{4}+d_{10}+d_{11}\right)$.

It is not difficult to compute the values of $\sum_{k=1}^{q^{2}} \chi_{50}(k)$. Using CHEVIE it is then easy to verify $\left(\sum_{k=1}^{q^{2}} B \chi_{50}(k), \sum_{k=1}^{q^{2}} B \chi_{50}(k)\right)_{B}=q^{2}$. Hence, the ${ }_{B} \chi_{50}(k)$ are $q^{2}$ different irreducible characters. 
Let ${ }_{B} \chi_{53}:={ }_{B} \chi_{2}(0) \cdot{ }_{B} \chi_{51}(0),{ }_{B} \chi_{54}:={ }_{B} \chi_{2}(0){ }_{B} \chi_{52}(0),{ }_{B} \chi_{55}:={ }_{B} \chi_{3}(0) \cdot{ }_{B} \chi_{51}(0)$, ${ }_{B} \chi_{56}:={ }_{B} \chi_{4}(0) \cdot{ }_{B} \chi_{51}(0),{ }_{B} \chi_{57}:={ }_{B} \chi_{3}(0) \cdot{ }_{B} \chi_{52}(0),{ }_{B} \chi_{58}:={ }_{B} \chi_{4}(0) \cdot{ }_{B} \chi_{52}(0)$.

Finally, we construct the irreducible characters ${ }_{B} \chi_{34},{ }_{B} \chi_{35}(k),{ }_{B} \chi_{36}(k),{ }_{B} \chi_{37}(k)$, which is the most complicated part in computing the character table of $B$. The characters ${ }_{B} \chi_{i}$ for $i \in\{1,2, \ldots, 22\} \cup\{26,27, \ldots, 33\}$ are $q^{4}+7 q^{2}+12$ different irreducible characters of $B$ and have the normal subgroup $U_{7} U_{9} U_{10} U_{11} U_{12} \unlhd B$ in their kernel, and hence, we can identify these characters with irreducible characters of $\bar{B}:=B / U_{7} U_{9} U_{10} U_{11} U_{12}$. The characters ${ }_{B} \chi_{34},{ }_{B} \chi_{35}(k),{ }_{B} \chi_{36}(k),{ }_{B} \chi_{37}(k)$ will be the remaining irreducible characters of $B$ with $U_{7} U_{9} U_{10} U_{11} U_{12}$ in their kernel. So, with the above identification, we want to construct the remaining irreducible characters of $\bar{B}$. We proceed in several steps:

Step 1: The number of the missing irreducible characters.

Using the relations in Tables 1 and 2 , we see that $\bar{B}$ has exactly $q^{4}+10 q^{2}+8$ conjugacy classes. Hence, there are exactly $3 q^{2}-4$ irreducible characters of $\bar{B}$ missing. We use Clifford theory to construct these.

Step 2: Preparations for Clifford theory.

The group $\bar{U}:=U_{2} U_{5} U_{6} U_{8} U_{7} U^{9} / U_{7} U^{9}$ is an elementary abelian normal subgroup of $\bar{B}$. So, $\bar{B}$ acts on $\bar{U}$ and $\operatorname{Irr}(\bar{U})$ by conjugation. Using the relations in Tables 1 and 2, it is not difficult to compute representatives for the orbits of $\bar{B}$ on $\operatorname{Irr}(\bar{U})$. In particular, we see that, for $x \in \mathbb{F}_{q^{2}}$, the characters

$$
\varphi_{x}: \bar{U} \rightarrow \mathbb{C}, \alpha_{2}\left(d_{2}\right) \alpha_{5}\left(d_{5}\right) \alpha_{6}\left(d_{6}\right) \alpha_{8}\left(d_{8}\right) U_{7} U^{9} \mapsto \phi\left(x \cdot d_{2}+d_{6}+d_{8}\right)
$$

are pairwise non-conjugate under the action of $\bar{B}$ and the missing irreducible characters of $\bar{B}$ are exactly those covering one of the $\varphi_{x}$ for $x \in \mathbb{F}_{q^{2}}$.

Step 3: Structure of the inertia subgroups.

All the $\varphi_{x}$ have the same inertia subgroup in $\bar{B}$, namely

$$
\bar{H}:=U_{1} U_{2} U_{4} U_{5} U_{6} U_{8} U_{7} U^{9} / U_{7} U^{9} .
$$

Using the relations in Tables 1 and 2, we see that $\bar{H}$ is a special 2-group with center $Z(\bar{H})=\bar{U}$. So we want to find the irreducible characters of $\bar{H}$ covering one of the $\varphi_{x}$. We use the theory of character triples (see [12, Chapter 11]).

Step 4: Number of $\varphi_{x}$ which are covered by 1 or 4 irreducibles of $\bar{H}$, resp.

Using [12, Exercise (11.10), (11.12) (c) and (11.15) (b)], we see that for every $x \in \mathbb{F}_{q^{2}}$ there are exactly 1,2 or 4 irreducible characters of $\bar{H}$ covering $\varphi_{x}$. Since $\varphi_{x}^{\bar{H}}$ vanishes outside of $\bar{U}$ we can compute the values of the induced character $\varphi_{x}^{\bar{H}}$ explicitly and can verify $\left(\varphi_{x}^{\bar{H}}, \varphi_{x}^{\bar{H}}\right)_{\bar{H}}=q^{4}$ for all $x \in \mathbb{F}_{q^{2}}$.

Suppose there is $x \in \mathbb{F}_{q^{2}}$ such that there are exactly 2 irreducible characters of $\bar{H}$ covering $\varphi_{x}$. Let $\chi_{1}, \chi_{2} \in \operatorname{Irr}(\bar{H})$ be these two characters. By [12, Exercise (6.2)] there is $f \in \mathbb{N}$ such that $\varphi_{x}^{\bar{H}}=f \cdot\left(\chi_{1}+\chi_{2}\right)$ and thus $\left(\varphi_{x}^{\bar{H}}, \varphi_{x}^{\bar{H}}\right)_{\bar{H}}=2 \cdot f^{2}$. This is a contradiction, since 2 occurs with even multiplicity in $q^{4}$, but with odd multiplicity in $2 \cdot f^{2}$.

This shows: For every $x \in \mathbb{F}_{q^{2}}$ there are exactly 1 or 4 irreducible characters of $\bar{H}$ covering $\varphi_{x}$. Since we already know that there are exactly $3 q^{2}-4$ irreducible characters of $\bar{H}$ covering one of the $\varphi_{x}$, it follows that there are $\frac{q^{2}+4}{3}$ elements $x \in \mathbb{F}_{q^{2}}$ such that there is exactly one irreducible character of $\bar{H}$ covering $\varphi_{x}$ and 
there are $\frac{2 q^{2}-4}{3}$ elements $x \in \mathbb{F}_{q^{2}}$ such that are exactly 4 irreducible characters of $\bar{H}$ covering $\varphi_{x}$.

Step 5: $\varphi_{0}$.

Again using [12, Exercises (11.10), (11.12) (c) and (11.15) (b)], we see that $\varphi_{0}$ is one of the irreducible characters of $\bar{U}$ which is covered by exactly one irreducible character of $\bar{H}$.

Step 6: Families of characters.

The group $L:=\left\langle T, U_{3}, n_{a}\right\rangle$ acts on $\operatorname{Irr}(\bar{H})$ and $\operatorname{Irr}(\bar{U})$ by conjugation. For abbreviation, let $S:=\left\{\varphi_{x} \mid x \in \mathbb{F}_{q^{2}}\right\} \subseteq \operatorname{Irr}(\bar{U})$. Using the relations in Tables $1-3$, it is not difficult to see that the characters $\varphi_{x}$ in $S$ belong to four different orbits under the action of $L$, say $\mathcal{O}_{0}, \mathcal{O}_{1}, \mathcal{O}_{2}, \mathcal{O}_{3}$ and we can choose the notation such that $S \cap \mathcal{O}_{0}=\left\{\varphi_{0}\right\},\left|S \cap \mathcal{O}_{1}\right|=\frac{q^{2}-2}{6},\left|S \cap \mathcal{O}_{2}\right|=\frac{q^{2}}{2}-1,\left|S \cap \mathcal{O}_{3}\right|=\frac{q^{2}+1}{3}$. The cardinalities $\left|S \cap \mathcal{O}_{i}\right|, i=0,1,2,3$, the results of Step 4 and the fact that $\varphi_{0}$ is covered by exactly one irreducible character of $\bar{H}$ imply that the characters in $S \cap \mathcal{O}_{3}$ and $\varphi_{0}$ are the only ones of the $\varphi_{x}$ which are covered by exactly one irreducible character of $\bar{H}$. Each of the remaining $\varphi_{x}$ is covered by four irreducible characters of $\bar{H}$. (Note that for $q^{2}=8$, we have $\left|S \cap \mathcal{O}_{2}\right|=\left|S \cap \mathcal{O}_{3}\right|$. In this case we choose our notation so that the $\varphi_{x} \in S \cap \mathcal{O}_{3}$ are covered by exactly one irreducible character of $\bar{H}$.)

Step 7: Construction of characters.

We have seen in Step 6: For every $\varphi_{x} \in \mathcal{O}_{0} \cup \mathcal{O}_{3}$ the induced character $\varphi_{x}^{\bar{H}}$ has exactly one irreducible constituent, say $\chi_{x}$. Thus, there is $f \in \mathbb{N}$ with $\varphi_{x}^{\bar{H}}=f \cdot \chi_{x}$. Then we have $q^{4}=\left(\varphi_{x}^{\bar{H}}, \varphi_{x}^{\bar{H}}\right)_{\bar{H}}=f^{2}$ and hence, $f=q^{2}$. Since $\bar{H}$ is the inertia subgroup of $\varphi_{x}$ in $\bar{B}$ Clifford theory tells us that $\frac{1}{q^{2}} \varphi_{x}^{\bar{B}}$ is the only irreducible character of $\bar{B}$ covering $\varphi_{x}$. Number the elements $x \in \mathbb{F}_{q^{2}}$ with $\varphi_{x} \in \mathcal{O}_{3}$ in some way, say $x_{1}, x_{2}, \ldots, x_{\left(q^{2}+1\right) / 3}$. Let ${ }_{B} \chi_{34}$ be the inflation of $\frac{1}{q^{2}} \varphi_{0}^{\bar{B}}$ to $B$ and let ${ }_{B} \chi_{37}(k)$, $k=1,2, \ldots,\left(q^{2}+1\right) / 3$, be the inflation of $\frac{1}{q^{2}} \varphi_{x_{k}}^{\bar{B}}$ to $B$.

The remaining irreducible characters of $\bar{B}$ and $B$, respectively, can be constructed similarly: For every $\varphi_{x} \in \mathcal{O}_{1} \cup \mathcal{O}_{2}$ the induced character $\varphi_{x}^{\bar{H}}$ has exactly four irreducible constituents, say $\chi_{x, 1}, \ldots, \chi_{x, 4}$. Since $\bar{H} / \bar{U}$ is abelian, we can apply [12, Exercise (6.2)], which implies that there is $f \in \mathbb{N}$ with $\varphi_{x}^{\bar{H}}=f \cdot\left(\chi_{x, 1}+\chi_{x, 2}+\right.$ $\left.\chi_{x, 3}+\chi_{x, 4}\right)$. Then we have $q^{4}=\left(\varphi_{x}^{\bar{H}}, \varphi_{x}^{\bar{H}}\right)_{\bar{H}}=4 \cdot f^{2}$ and hence, $f=\frac{q^{2}}{2}$. Since $\bar{H}$ is the inertia subgroup of $\varphi_{x}$ in $\bar{B}$, Clifford theory tells us that $\frac{2}{q^{2}} \varphi_{x}^{\bar{B}}$ is a sum of four different irreducible characters of $\bar{B}$ and these are the only irreducible characters of $\bar{B}$ covering $\varphi_{x}$.

Label the irreducible constituents of the $\varphi_{x}^{\bar{B}}$ with $\varphi_{x} \in \mathcal{O}_{1}$ in some way, say $\bar{B} \chi_{35}(k), k=1,2, \ldots, 4 \cdot\left(q^{2}-2\right) / 6$. Analogously, label the irreducible constituents of the $\varphi_{x}^{\bar{B}}$ with $\varphi_{x} \in \mathcal{O}_{2}$ in some way, say ${ }_{B} \chi_{36}(k), k=1,2, \ldots, 4 \cdot\left(q^{2}-2\right) / 2$. By inflation, we get the irreducible characters ${ }_{B} \chi_{35}(k), k=1,2, \ldots, 4 \cdot\left(q^{2}-2\right) / 6$ and ${ }_{B} \chi_{36}(k), k=1,2, \ldots, 4 \cdot\left(q^{2}-2\right) / 2$ of $B$.

Step 8: Computation of character values.

The values of ${ }_{B} \chi_{34}$ can be computed directly using only the definition of induced characters and (1) and (2). The values of ${ }_{B} \chi_{35}(k),{ }_{B} \chi_{36}(k),{ }_{B} \chi_{37}(k)$ on the unipotent classes $c_{1,46}(a), c_{1,47}(a)$ and $c_{1,48}(a)$ depend on the parameter $a$ and we do 
not have a generic description of these classes. But, using orthogonality relations applied to $\bar{B}$, it is possible to determine the values of the sums $\sum_{k=1}^{4 \cdot\left(q^{2}-2\right) / 6}{ }_{B} \chi_{35}(k)$, $\sum_{k=1}^{4 \cdot\left(q^{2}-2\right) / 2}{ }_{B} \chi_{36}(k), \sum_{k=1}^{\left(q^{2}+1\right) / 3}{ }_{B} \chi_{37}(k)$. This completes the construction of the irreducible characters of $B$.

THEOREM 4.2. The character table of the Borel subgroup B is given by Tables A.5 and A.6 in the Appendix.

Proof. Computing scalar products with CHEVIE we see that ${ }_{B} \chi_{1}(k, l), \ldots,{ }_{B} \chi_{58}$ are $q^{4}+22 q^{2}+13$ irreducible and pairwise different characters.

We point out that we are not able to describe all values of all irreducible characters of $B$. This seems to be a usual phenomenon for generic character tables of parabolic subgroups (see for example [5]). For example: we cannot describe all values of ${ }_{B} \chi_{25}(k)$ generically because an inspection of small values of $q$ shows, that the values of ${ }_{B} \chi_{25}(k)$ on the classes $c_{1,34}(a), c_{1,35}(a), c_{1,36}(a), c_{1,37}(a)$ depend on the parameter $a$ and we do not have a generic description of these classes. For fixed $q$ (not too large), there is no difficulty in computing the values of all irreducible characters of $B$ using only the above definition of the characters.

\section{M-groups and characters of Sylow-2-subgroups}

In this section we show that the Borel subgroup $B$ is an $M$-group and determine the degrees of the irreducible characters of a Sylow-2-subgroup of ${ }^{2} F_{4}\left(q^{2}\right)$.

THEOREM 5.1. The Borel subgroup $B$ is an $M$-group, that means, all irreducible characters of $B$ can be obtained by inducing linear characters from suitable subgroups.

Proof. We show that ${ }_{B} \chi_{3}(k)$ is monomial, i.e., induced by a linear character. By construction $U^{3} \subseteq \operatorname{ker}\left({ }_{B} \chi_{3}(k)\right)$, so we can identify ${ }_{B} \chi_{3}(k)$ with an irreducible character of the factor group $\bar{B}:=B / U^{3}$. The group $\bar{B}$ is a direct product $\bar{B} \cong$ $C_{T}\left(\alpha_{1}(1) \alpha_{2}(1)\right) \times B_{\mathrm{Sz}}$ where $C_{T}\left(\alpha_{1}(1) \alpha_{2}(1)\right) \cong \mathbb{Z}_{q^{2}-1}$ and $B_{\mathrm{Sz}}$ is a group of order $q^{4}\left(q^{2}-1\right)$ isomorphic to a Borel subgroup of $\mathrm{Sz}\left(q^{2}\right)$. So there is a linear character $\lambda_{k} \in \operatorname{Irr}\left(C_{T}\left(\alpha_{1}(1) \alpha_{2}(1)\right)\right)$ and $\psi \in \operatorname{Irr}\left(B_{\mathrm{Sz}}\right)$ such that ${ }_{B} \chi_{3}(k)(h b)=\lambda_{k}(h) \psi(b)$ for all $h \in C_{T}\left(\alpha_{1}(1) \alpha_{2}(1)\right)$ and $b \in B_{\mathrm{Sz}}$. In particular, $\psi$ has degree $\frac{q}{\sqrt{2}}\left(q^{2}-1\right)$. We have $B_{\mathrm{Sz}} \cong \mathbb{Z}_{q^{2}-1} \ltimes \bar{U}$ where $\bar{U}$ is a group of order $q^{4}$. Clifford theory (see [12, Theorem (6.11), Corollary (6.17), Corollary (6.28)]) implies that $\psi$ is induced by an irreducible character of degree $\frac{q}{\sqrt{2}}$ of $\bar{U}$. Since $\bar{U}$ is a 2-group and hence an $M$ group we get that $\psi$ and then ${ }_{B} \chi_{3}(k)$ are monomial. Analogously, $B \chi_{4}(k),{ }_{B} \chi_{26}(k)$, ${ }_{B} \chi_{27}(k),{ }_{B} \chi_{44}(k),{ }_{B} \chi_{45}(k),{ }_{B} \chi_{51}(k),{ }_{B} \chi_{52}(k)$ are induced by linear characters.

By definition, $B \chi_{6}={ }_{B} \chi_{3}(0) \cdot{ }_{B} \chi_{5}(0)=\varphi^{B} \cdot \varphi^{B}$ where $\varphi$ is a character of the group $C_{T}\left(\alpha_{1}(1) \alpha_{2}(1)\right) U$ and $\varphi^{\prime}$ is a character of $C_{T}\left(\alpha_{3}(1)\right) U$. Mackey's tensor product decomposition [14, Corollary II.6.4] implies that ${ }_{B} \chi_{6}$ is induced by an irreducible character of degree $\frac{q}{\sqrt{2}}$ of $U=C_{T}\left(\alpha_{1}(1) \alpha_{2}(1)\right) U \cap C_{T}\left(\alpha_{3}(1)\right) U$. Since $U$ is a 2-group and hence an $M$-group it follows that ${ }_{B} \chi_{6}$ is induced by a linear character of some subgroup of $U$. Analogously, $B \chi_{7}, B \chi_{28}, B \chi_{29}, \ldots, B \chi_{33}, B \chi_{46}$, ${ }_{B} \chi_{47}, B \chi_{53}, B \chi_{54}, \ldots, B \chi_{58}$ are induced by linear characters. 
The characters ${ }_{B} \chi_{34},{ }_{B} \chi_{35}(k),{ }_{B} \chi_{36}(k),{ }_{B} \chi_{37}(k)$ are induced from the subgroup $U_{1} U_{2} U_{4} U_{5} U_{6} U^{8}$ which is a 2-group. Similarly, ${ }_{B} \chi_{48}(k),{ }_{B} \chi_{49}(k)$ are induced from the subgroup $U_{3} U_{4} U_{5} U_{6} U^{8}$. Therefore, all these characters are monomial.

The remaining irreducible characters of $B$ are monomial by construction.

Theorem 5.2. The degrees of the irreducible characters of the Sylow-2-subgroup U of ${ }^{2} F_{4}\left(q^{2}\right)$ are given by Table 7. In particular, the number of conjugacy classes of $U$ is $8 q^{6}+12 q^{4}-32 q^{2}+13$.

Table 7: Degrees of the irreducible characters of $U$.

\begin{tabular}{cl|cl}
\hline Degree & $\begin{array}{l}\text { Number of irreducible } \\
\text { characters }\end{array}$ & Degree & $\begin{array}{l}\text { Number of irreducible } \\
\text { characters }\end{array}$ \\
\hline 1 & $q^{4}$ & $q^{4}$ & $\frac{1}{3}\left(4 q^{6}-q^{4}-q^{2}-2\right)$ \\
$\frac{q}{\sqrt{2}}$ & $2 q^{4}-2 q^{2}$ & $q^{6}$ & $q^{6}-2 q^{2}+1$ \\
$q^{2}$ & $q^{4}-q^{2}$ & $\frac{q^{7}}{\sqrt{2}}$ & $2 q^{6}-2 q^{4}$ \\
$\frac{q^{3}}{\sqrt{2}}$ & $2 q^{4}-2 q^{2}$ & $q^{8}$ & $q^{6}-q^{4}$ \\
$\frac{q^{4}}{4}$ & $8 q^{4}-16 q^{2}+8$ & $\frac{q^{9}}{\sqrt{2}}$ & $2 q^{4}-2 q^{2}$ \\
$\frac{q^{4}}{2}$ & $\frac{1}{3}\left(8 q^{6}-14 q^{4}+4 q^{2}+2\right)$ & $\frac{q^{10}}{2}$ & $4 q^{4}-8 q^{2}+4$ \\
\hline
\end{tabular}

Proof. The torus $T$ acts on the set of conjugacy classes of $U$ and on $\operatorname{Irr}(U)$ by conjugation and the number of orbits of $T$ on each of these sets is $8 q^{2}+45$ (this follows from the fact that $B$ has exactly $8 q^{2}+45$ unipotent conjugacy classes, see Table A.4 in the Appendix, and Brauer's Permutation Lemma [11, Satz V.13.5]). Let $\psi \in \operatorname{Irr}(U), I_{\psi}$ be the inertia subgroup of $\psi$ in $B$ and $\chi \in \operatorname{Irr}(B)$ an irreducible character of $B$ covering $\psi$. By Clifford theory [12, Theorems (6.11) and (6.28), Corollary (6.17)] we know that the 2-part of the degree $\chi(1)$ is equal to the degree $\psi(1)$ and that the $2^{\prime}$-part of the degree $\chi(1)$ is equal to $\left[B: I_{\psi}\right]$, the size of the orbit of $\psi$ under the action of $T$. The claim now follows from Table A.6 in the Appendix and the fact that the number of orbits of $T$ on $\operatorname{Irr}(U)$ is $8 q^{2}+45$.

\section{McKay conjecture for ${ }^{2} F_{4}\left(q^{2}\right)$}

In [13], Isaacs, Malle and Navarro reduced the McKay conjecture to a question about finite simple groups. They showed that the conjecture is true for every finite group if every finite non-abelian simple group satisfies certain conditions. In this section, we prove that these conditions hold for $G={ }^{2} F_{4}\left(q^{2}\right)$.

Let $O=\operatorname{Out}(G)$. Then $O=\langle\alpha\rangle$ and $\operatorname{Aut}(G)=G \rtimes\langle\alpha\rangle$, where $\alpha$ is a field automorphism of order $2 n+1$. We write $\operatorname{Irr}_{2^{\prime}}(B)$ and $\operatorname{Irr}_{2^{\prime}}(G)$ for the set of irreducible characters of odd degree of $B$ and $G$, respectively. Since $B$ is $\alpha$-invariant we get an action of $O$ on $\operatorname{Irr}_{2^{\prime}}(B)$ and $\operatorname{Irr}_{2^{\prime}}(G)$. Our main task is to show that $\operatorname{Irr}_{2^{\prime}}(B)$ and $\operatorname{Irr}_{2^{\prime}}(G)$ are isomorphic $O$-sets. Our approach is similar to that in [10]: we want to use [12, Lemma (13.23)], so we have to count fixed points of subgroups $H \leqslant O$.

Lemma 6.1. Let $t \mid 2 n+1$ and $H=\left\langle\alpha^{t}\right\rangle$. The number of fixed points of $\operatorname{Irr}_{2^{\prime}}(B)$ under the action of $H$ is $2^{2 t}$. 
Proof. We get $\operatorname{Irr}_{2^{\prime}}(B)=\left\{B \chi_{1}(k, l)\right\} \cup\left\{{ }_{B} \chi_{2}(k)\right\} \cup\left\{{ }_{B} \chi_{5}(k)\right\} \cup\left\{{ }_{B} \chi_{8}\right\}$ from Table A.5 in the Appendix. From Table A.6 in the Appendix we see: $B \chi_{1}(k, l)^{\alpha^{t}}=$ ${ }_{B} \chi_{1}\left(2^{t} k, 2^{t} l\right)$ where we interpret the character parameters $k, l$ modulo $q^{2}-1$ (see the remarks on character parameter groups in Section 3.7 of the CHEVIE manual). So we get: ${ }_{B} \chi_{1}(k, l)^{\alpha^{t}}={ }_{B} \chi_{1}(k, l)$ if and only if $2^{t} k \equiv k$ and $2^{t} l \equiv l \bmod q^{2}-1$. This is equivalent with the fact that $k, l$ are multiples of $\frac{q^{2}-1}{2^{t}-1}$. So the number of fixed points of $H$ on $\left\{{ }_{B} \chi_{1}(k, l)\right\}$ is $\left(2^{t}-1\right)^{2}$. Similarly, the number of fixed points of $H$ on $\left\{{ }_{B} \chi_{2}(k)\right\}$ is $2^{t}-1$ and the same holds for the fixed points on $\left\{{ }_{B} \chi_{5}(k)\right\}$. Finally, we see from the character table of $B$ that ${ }_{B} \chi_{8}$ is fixed by $H$. So the number of fixed points of $\operatorname{Irr}_{2^{\prime}}(B)$ under the action of $H$ is: $\left(2^{t}-1\right)^{2}+2\left(2^{t}-1\right)+1=2^{2 t}$.

Lemma 6.2. Let $t \mid 2 n+1$ and $H=\left\langle\alpha^{t}\right\rangle$. The number of fixed points of $\operatorname{Irr}_{2^{\prime}}(G)$ under the action of $H$ is $2^{2 t}$.

Proof. Let $\operatorname{Irr}^{s s}(G)$ denote the set of semisimple irreducible characters of $G$. From the character table of ${ }^{2} F_{4}\left(q^{2}\right)$ in the CHEVIE library we see that $\operatorname{Irr}_{2^{\prime}}(G)=$ $\operatorname{Irr}^{s s}(G)$. Let $\mathcal{S}(G)$ be the set of all semisimple conjugacy classes of $G$.

We can assume that $\alpha$ is the restriction of the endomorphism of the algebraic group $\mathbf{G}$ which is given by $x_{r}(t) \mapsto x_{r}\left(t^{2}\right)$ (which we also denote by $\alpha$ ). Since $\alpha$ raises every element of the maximally split torus $\mathbf{T}$ to its 2 nd power we see that $\alpha^{t}$ acts on the semisimple conjugacy classes of $G$ like the $2^{t}$ th power map (this does not mean, that $\alpha^{t}$ maps every semisimple element of $G$ to its $2^{t}$ th power).

Since $G={ }^{2} F_{4}\left(q^{2}\right)$ is isomorphic to its dual group (in the sense of [3], Section 4.4, p. 120), the number of fixed points of $\alpha^{t}$ on $\operatorname{Irr}_{2^{\prime}}(G)=\operatorname{Irr}^{s s}(G)$ is equal to the number $\left|\mathcal{S}(G)^{\alpha^{t}}\right|$ of fixed points of $\alpha^{t}$ on $\mathcal{S}(G)$. By Remark (a) after [1, Lemma 4.1], we have $\left|\mathcal{S}(G)^{\alpha^{t}}\right|=\left|\mathcal{S}\left({ }^{2} F_{4}\left(2^{2 t}\right)\right)\right|$. From [3, Theorem 3.7.6] we get $\left|\mathcal{S}\left({ }^{2} F_{4}\left(2^{2 t}\right)\right)\right|=$ $2^{2 t}$.

TheOREM 6.3. For $q^{2}=2^{2 n+1} \geqslant 8$, the group ${ }^{2} F_{4}\left(q^{2}\right)$ is good for the prime 2 in the sense of $[13$, Section 10].

Proof. Note that ${ }^{2} F_{4}\left(q^{2}\right)$ has trivial Schur multiplier. Let $A:=B \rtimes\langle\alpha\rangle$ (where $\alpha$ is the field automorphism of $G$ as before). From Lemmas 6.1, 6.2 and [12, (13.23)], we know that there is an $A$-equivariant bijection ()$^{*}: \operatorname{Irr}_{2^{\prime}}(G) \rightarrow \operatorname{Irr}_{2^{\prime}}(B)$. The verification of the remaining properties for ${ }^{2} F_{4}\left(q^{2}\right)$ to be good is analogous to the proof of [10, Theorem 6.1].

In particular, the McKay conjecture for $p=2$ is true for ${ }^{2} F_{4}\left(q^{2}\right)$.

\section{Acknowledgments}

Part of this work was done during a visit of the first author at the Department of Mathematics of the University of Auckland in September 2007. He wishes to express his sincere thanks to all the persons of the department for their hospitality, and also to the Marsden Fund of New Zealand who supported his visit. The second author wishes to express his appreciation to the Foundation for Research, Science and Technology of New Zealand for supporting his research from 2005 to 2008, and also to the Japan Society for the Promotion of Science (JSPS) for supporting his research from 2008 to 2010. 
Appendix A.

Table A.1 (cf. Shinoda [19, Table IV]): Parametrization of those semisimple conjugacy classes of ${ }^{2} F_{4}\left(q^{2}\right)$ having non-empty intersection with one of the proper parabolic subgroups of ${ }^{2} F_{4}\left(q^{2}\right)$.

\begin{tabular}{|c|c|c|}
\hline Representative & Parameters & $\begin{array}{l}\text { Number of } \\
\text { Classes }\end{array}$ \\
\hline$h_{1}:=h(1,1,1,1)$ & & 1 \\
\hline$h_{2}(i):=h\left(1,1, \tilde{\zeta}_{2}^{i}, \tilde{\zeta}_{2}^{(2 \theta-1) i}\right)$ & $\begin{array}{l}i=0, \ldots, q^{2}-2 \\
i \neq 0\end{array}$ & $\frac{q^{2}-2}{2}$ \\
\hline$h_{3}(i):=h\left(\tilde{\zeta}_{2}^{i}, \tilde{\zeta}_{2}^{(2 \theta-1) i}, \tilde{\zeta}_{2}^{(2 \theta+1) i}, \tilde{\zeta}_{2}^{i}\right)$ & $\begin{array}{l}i=0, \ldots, q^{2}-2 \\
i \neq 0\end{array}$ & $\frac{q^{2}-2}{2}$ \\
\hline$h_{4}(i, j):=h\left(\tilde{\zeta}_{2}^{i}, \tilde{\zeta}_{2}^{(2 \theta-1) i}, \tilde{\zeta}_{2}^{j}, \tilde{\zeta}_{2}^{(2 \theta-1) j}\right)$ & $\begin{array}{l}i=0, \ldots, q^{2}-2 ; j=0, \ldots, q^{2}-2 \\
\text { many exceptions }\end{array}$ & $\frac{q^{4}-10 q^{2}+16}{16}$ \\
\hline$h_{5}:=h\left(\tilde{\varepsilon}_{3}, \tilde{\varepsilon}_{3}^{1-\theta}, \tilde{\varepsilon}_{3}^{-1-\theta}, \tilde{\varepsilon}_{3}^{-\theta}\right)$ & & 1 \\
\hline$h_{6}(i):=h\left(\tilde{\xi}_{2}^{i}, \tilde{\xi}_{2}^{(1-\theta) i}, \tilde{\xi}_{2}^{(-1-\theta) i}, \tilde{\xi}_{2}^{-\theta i}\right)$ & $\begin{array}{l}i=0, \ldots, q^{2} \\
i \neq 0, \frac{q^{2}+1}{3}, \frac{2\left(q^{2}+1\right)}{3}\end{array}$ & $\frac{q^{2}-2}{2}$ \\
\hline $\begin{aligned} & h_{7}(i):=h\left(\tilde{\zeta}_{4}^{\left(4 \theta^{3}+2 \theta^{2}+1\right) i},\right. \tilde{\zeta}_{4}^{\left(2 \theta^{2}+2 \theta-1\right) i}, \\
&\left.\tilde{\zeta}_{4}^{\left(-2 \theta^{2}+2 \theta+1\right) i}, \tilde{\zeta}_{4}^{\left.-4 \theta^{3}+2 \theta^{2}+1\right) i}\right)\end{aligned}$ & $\begin{array}{l}i=0, \ldots, q^{4}-2 \\
\quad \begin{array}{l}i \neq\left(q^{2}-1\right) l, \quad l=0, \ldots, q^{2} \\
i \neq\left(q^{2}+1\right) l, \quad l=0, \ldots, q^{2}-2\end{array}\end{array}$ & $\frac{q^{4}-2 q^{2}}{4}$ \\
\hline$h_{8}(i):=h\left(1,1, \tilde{\varphi}_{8}^{\prime \prime i}, \tilde{\varphi}_{8}^{\prime \prime-q^{2} i}\right)$ & $\begin{array}{l}i=0, \ldots, q^{2}-\sqrt{2} q \\
i \neq 0\end{array}$ & $\frac{q^{2}-\sqrt{2} q}{4}$ \\
\hline $\begin{array}{c}h_{9}(i):=h\left(\tilde{\psi}_{8}^{\prime \prime\left(2 \theta^{2}-2 \theta+1\right) i}, \tilde{\psi}_{8}^{\prime \prime\left(4 \theta^{3}-6 \theta^{2}+4 \theta-1\right) i},\right. \\
\left.\tilde{\psi}_{8}^{\prime \prime\left(2 \theta^{2}-1\right) i}, \tilde{\psi}_{8}^{\prime \prime(}\left(-4 \theta^{4}+2 \theta^{2}\right) i\right)\end{array}$ & $\begin{array}{l}i=0, \ldots, q^{4}-\sqrt{2} q^{3}+\sqrt{2} q-2 \\
i \neq\left(q^{2}-1\right) l, \quad l=0, \ldots, q^{2}-\sqrt{2} q \\
i \neq\left(q^{2}-\sqrt{2} q+1\right) l, \quad l=0, \ldots, q^{2}-2\end{array}$ & $\begin{array}{l}\frac{1}{8}\left(q^{4}-\sqrt{2} q^{3}\right. \\
\left.-2 q^{2}+2 \sqrt{2} q\right)\end{array}$ \\
\hline$h_{10}(i):=h\left(1,1, \tilde{\varphi}_{8}^{\prime i}, \tilde{\varphi}_{8}^{\prime-q^{2} i}\right)$ & $\begin{array}{l}i=0, \ldots, q^{2}+\sqrt{2} q \\
i \neq 0\end{array}$ & $\frac{q^{2}+\sqrt{2} q}{4}$ \\
\hline $\begin{array}{c}h_{11}(i):=h\left(\tilde{\psi}_{8}^{\prime\left(2 \theta^{2}+2 \theta+1\right) i}, \tilde{\psi}_{8}^{\prime\left(4 \theta^{3}+2 \theta^{2}-1\right) i},\right. \\
\left.\tilde{\psi}_{8}^{\prime\left(2 \theta^{2}-1\right) i}, \tilde{\psi}_{8}^{\prime\left(-4 \theta^{4}+2 \theta^{2}\right) i}\right)\end{array}$ & $\begin{array}{l}i=0, \ldots, q^{4}+\sqrt{2} q^{3}-\sqrt{2} q-2 \\
i \neq\left(q^{2}-1\right) l, \quad l=0, \ldots, q^{2}+\sqrt{2} q \\
i \neq\left(q^{2}+\sqrt{2} q+1\right) l, \quad l=0, \ldots, q^{2}-2\end{array}$ & $\begin{array}{l}\frac{1}{8}\left(q^{4}+\sqrt{2} q^{3}\right. \\
\left.-2 q^{2}-2 \sqrt{2} q\right)\end{array}$ \\
\hline
\end{tabular}

Table A.2 (K. Shinoda [19, Tables II, V]): Those conjugacy classes of ${ }^{2} F_{4}\left(q^{2}\right)$ having non-empty intersection with one of the proper parabolic subgroups of ${ }^{2} F_{4}\left(q^{2}\right)$. (For a definition of the element $\zeta \in \mathbb{F}_{q^{2}}$ occurring in the representative for $c_{1,9}$ and the group elements $x, x^{\prime}, x^{\prime \prime}$ in the representatives for $c_{5,2}, c_{5,3}, c_{5,4}$ respectively, see Section 3. Note that $x, x^{\prime}, x^{\prime \prime}$ depend on the congruence class of $\theta \bmod 3$.)

\begin{tabular}{lll}
\hline Notation & Representative & $\left|C_{2} F_{4}\left(q^{2}\right)\right|$ \\
\hline$c_{1,0}$ & 1 & $q^{24}\left(q^{12}+1\right)\left(q^{8}-1\right)\left(q^{6}+1\right)\left(q^{2}-1\right)$ \\
$c_{1,1}$ & $\alpha_{12}(1)$ & $q^{24}\left(q^{4}+1\right)\left(q^{2}-1\right)$ \\
$c_{1,2}$ & $\alpha_{10}(1)$ & $q^{20}\left(q^{4}-1\right)$ \\
$c_{1,3}$ & $\alpha_{5}(1) \alpha_{12}(1)$ & $2 q^{14}\left(q^{4}+1\right)\left(q^{2}-1\right)$ \\
$c_{1,4}$ & $\alpha_{5}(1)$ & $2 q^{14}\left(q^{4}+1\right)\left(q^{2}-1\right)$ \\
$c_{1,5}$ & $\alpha_{7}(1) \alpha_{8}(1)$ & $q^{16}$ \\
$c_{1,6}$ & $\alpha_{5}(1) \alpha_{7}(1)$ & $q^{14}$ \\
\hline
\end{tabular}


Character table of a Borel subgroup of the Ree groups ${ }^{2} F_{4}\left(q^{2}\right)$

Table A.2 (continued)

\begin{tabular}{|c|c|c|}
\hline Notation & Representative & $\left|C_{2} F_{4}\left(q^{2}\right)\right|$ \\
\hline$c_{1,7}$ & $\alpha_{5}(1) \alpha_{6}(1)$ & $6 q^{12}$ \\
\hline$c_{1,8}$ & $\alpha_{5}(1) \alpha_{6}(1) \alpha_{8}(1)$ & $2 q^{12}$ \\
\hline$c_{1,9}$ & $\alpha_{2}(1) \alpha_{6}(\zeta) \alpha_{8}(1)$ & $3 q^{12}$ \\
\hline$c_{1,10}$ & $\alpha_{2}(1) \alpha_{4}(1)$ & $2 q^{8}$ \\
\hline$c_{1,11}$ & $\alpha_{2}(1) \alpha_{4}(1) \alpha_{5}(1)$ & $4 q^{8}$ \\
\hline$c_{1,12}$ & $\alpha_{2}(1) \alpha_{4}(1) \alpha_{5}(1) \alpha_{8}(1)$ & $4 q^{8}$ \\
\hline$c_{1,13}$ & $\alpha_{2}(1) \alpha_{3}(1)$ & $2 q^{6}$ \\
\hline$c_{1,14}$ & $\alpha_{2}(1) \alpha_{3}(1) \alpha_{5}(1)$ & $2 q^{6}$ \\
\hline$c_{1,15}$ & $\alpha_{1}(1) \alpha_{3}(1)$ & $4 q^{4}$ \\
\hline$c_{1,16}$ & $\alpha_{1}(1) \alpha_{2}(1) \alpha_{3}(1)$ & $4 q^{4}$ \\
\hline$c_{1,17}$ & $\alpha_{1}(1) \alpha_{3}(1) \alpha_{5}(1)$ & $4 q^{4}$ \\
\hline$c_{1,18}$ & $\alpha_{1}(1) \alpha_{2}(1) \alpha_{3}(1) \alpha_{5}(1)$ & $4 q^{4}$ \\
\hline$c_{2,0}(i)$ & $h_{2}(i)$ & $q^{4}\left(q^{4}+1\right)\left(q^{2}-1\right)^{2}$ \\
\hline$c_{2,1}(i)$ & $h_{2}(i) \alpha_{12}(1)$ & $q^{4}\left(q^{2}-1\right)$ \\
\hline$c_{2,2}(i)$ & $h_{2}(i) \alpha_{5}(1)$ & $2 q^{2}\left(q^{2}-1\right)$ \\
\hline$c_{2,3}(i)$ & $h_{2}(i) \alpha_{5}(1) \alpha_{12}(1)$ & $2 q^{2}\left(q^{2}-1\right)$ \\
\hline$c_{3,0}(i)$ & $h_{3}(i)$ & $q^{2}\left(q^{2}+1\right)\left(q^{2}-1\right)^{2}$ \\
\hline$c_{3,1}(i)$ & $h_{3}(i) \alpha_{9}(1)$ & $q^{2}\left(q^{2}-1\right)$ \\
\hline$c_{4,0}(i, j)$ & $h_{4}(i, j)$ & $\left(q^{2}-1\right)^{2}$ \\
\hline$c_{5,0}$ & $h_{5}$ & $q^{6}\left(q^{6}+1\right)\left(q^{4}-1\right)$ \\
\hline$c_{5,1}$ & $h_{5} x_{17}(1) x_{22}(1)$ & $q^{6}\left(q^{2}+1\right)$ \\
\hline$c_{5,2}$ & $h_{5} x$ & $3 q^{4}$ \\
\hline$c_{5,3}$ & $h_{5} x^{\prime}$ & $3 q^{4}$ \\
\hline$c_{5,4}$ & $h_{5} x^{\prime \prime}$ & $3 q^{4}$ \\
\hline$c_{6,0}(i)$ & $h_{6}(i)$ & $q^{2}\left(q^{4}-1\right)\left(q^{2}+1\right)$ \\
\hline$c_{6,1}(i)$ & $h_{6}(i) x_{17}(1) x_{22}(1)$ & $q^{2}\left(q^{2}+1\right)$ \\
\hline$c_{7,0}(i)$ & $h_{7}(i)$ & $q^{4}-1$ \\
\hline$c_{8,0}(i)$ & $h_{8}(i)$ & $q^{4}\left(q^{2}-\sqrt{2} q+1\right)\left(q^{4}+1\right)\left(q^{2}-1\right)$ \\
\hline$c_{8,1}(i)$ & $h_{8}(i) x_{21}(1) x_{24}(1)$ & $q^{4}\left(q^{2}-\sqrt{2} q+1\right)$ \\
\hline$c_{8,2}(i)$ & $h_{8}(i) x_{8}(1) x_{16}(1) x_{21}(1)$ & $2 q^{2}\left(q^{2}-\sqrt{2} q+1\right)$ \\
\hline$c_{8,3}(i)$ & $h_{8}(i) x_{8}(1) x_{16}(1) x_{24}(1)$ & $2 q^{2}\left(q^{2}-\sqrt{2} q+1\right)$ \\
\hline$c_{9,0}(i)$ & $h_{9}(i)$ & $\left(q^{2}-\sqrt{2} q+1\right)\left(q^{2}-1\right)$ \\
\hline$c_{10,0}(i)$ & $h_{10}(i)$ & $q^{4}\left(q^{2}+\sqrt{2} q+1\right)\left(q^{4}+1\right)\left(q^{2}-1\right)$ \\
\hline$c_{10,1}(i)$ & $h_{10}(i) x_{21}(1) x_{24}(1)$ & $q^{4}\left(q^{2}+\sqrt{2} q+1\right)$ \\
\hline$c_{10,2}(i)$ & $h_{10}(i) x_{8}(1) x_{16}(1) x_{21}(1)$ & $2 q^{2}\left(q^{2}+\sqrt{2} q+1\right)$ \\
\hline$c_{10,3}(i)$ & $h_{10}(i) x_{8}(1) x_{16}(1) x_{24}(1)$ & $2 q^{2}\left(q^{2}+\sqrt{2} q+1\right)$ \\
\hline$c_{11,0}(i)$ & $h_{11}(i)$ & $\left(q^{2}+\sqrt{2} q+1\right)\left(q^{2}-1\right)$ \\
\hline
\end{tabular}


Table A.3: Parametrization of the semisimple conjugacy classes of $B$.

\begin{tabular}{|c|c|c|}
\hline Representative & Parameters & $\begin{array}{l}\text { Number of } \\
\text { Classes }\end{array}$ \\
\hline$h_{1}:=h(1,1,1,1)$ & & 1 \\
\hline$h_{2}(i):=h\left(\tilde{\zeta}_{2}^{i}, \tilde{\zeta}_{2}^{(2 \theta-1) i}, 1,1\right)$ & $\begin{array}{l}i=0, \ldots, q^{2}-2 \\
i \neq 0\end{array}$ & $q^{2}-2$ \\
\hline$h_{3}(i):=h\left(\tilde{\zeta}_{2}^{i}, \tilde{\zeta}_{2}^{(2 \theta-1) i}, \tilde{\zeta}_{2}^{(2 \theta-1) i}, \tilde{\zeta}_{2}^{\left(4 \theta^{2}-4 \theta+1\right) i}\right)$ & $\begin{array}{l}i=0, \ldots, q^{2}-2 \\
i \neq 0\end{array}$ & $q^{2}-2$ \\
\hline$h_{4}(i):=h\left(\tilde{\zeta}_{2}^{i}, \tilde{\zeta}_{2}^{(2 \theta-1) i}, \tilde{\zeta}_{2}^{i}, \tilde{\zeta}_{2}^{(2 \theta-1) i}\right)$ & $\begin{array}{l}i=0, \ldots, q^{2}-2 \\
i \neq 0\end{array}$ & $q^{2}-2$ \\
\hline$h_{5}(i):=h\left(1,1, \tilde{\zeta}_{2}^{i}, \tilde{\zeta}_{2}^{(2 \theta-1) i}\right)$ & $\begin{array}{l}i=0, \ldots, q^{2}-2 \\
i \neq 0\end{array}$ & $q^{2}-2$ \\
\hline$h_{6}(i):=h\left(\tilde{\zeta}_{2}^{i}, \tilde{\zeta}_{2}^{(2 \theta-1) i}, \tilde{\zeta}_{2}^{-i}, \tilde{\zeta}_{2}^{(1-2 \theta) i}\right)$ & $\begin{array}{l}i=0, \ldots, q^{2}-2 \\
i \neq 0\end{array}$ & $q^{2}-2$ \\
\hline$h_{7}(i):=h\left(\tilde{\zeta}_{2}^{i}, \tilde{\zeta}_{2}^{(2 \theta-1) i}, \tilde{\zeta}_{2}^{(1-2 \theta) i}, \tilde{\zeta}_{2}^{\left(-4 \theta^{2}+4 \theta-1\right) i}\right)$ & $\begin{array}{l}i=0, \ldots, q^{2}-2 \\
\quad i \neq 0\end{array}$ & $q^{2}-2$ \\
\hline$h_{8}(i):=h\left(\tilde{\zeta}_{2}^{(2 \theta-1) i}, \tilde{\zeta}_{2}^{\left(4 \theta^{2}-4 \theta+1\right) i}, \tilde{\zeta}_{2}^{i}, \tilde{\zeta}_{2}^{(2 \theta-1) i}\right)$ & $\begin{array}{l}i=0, \ldots, q^{2}-2 \\
i \neq 0\end{array}$ & $q^{2}-2$ \\
\hline$h_{9}(i):=h\left(\tilde{\zeta}_{2}^{(1-2 \theta) i}, \tilde{\zeta}_{2}^{\left(-4 \theta^{2}+4 \theta-1\right) i}, \tilde{\zeta}_{2}^{i}, \tilde{\zeta}_{2}^{(2 \theta-1) i}\right)$ & $\begin{array}{l}i=0, \ldots, q^{2}-2 \\
i \neq 0\end{array}$ & $q^{2}-2$ \\
\hline$h_{10}(i, j):=h\left(\tilde{\zeta}_{2}^{i}, \tilde{\zeta}_{2}^{(2 \theta-1) i}, \tilde{\zeta}_{2}^{j}, \tilde{\zeta}_{2}^{(2 \theta-1) j}\right)$ & $\begin{array}{l}i, j=0, \ldots, q^{2}-2 \\
i, j \neq 0 \\
j \neq \pm i, \pm(2 \theta-1) i \\
i \neq \pm(2 \theta-1) j\end{array}$ & $q^{4}-10 q^{2}+16$ \\
\hline
\end{tabular}

Table A.4: The conjugacy classes of $B$. (The parameter $a$ in the representatives for the conjugacy classes of type $c_{1,29}, c_{1,30}, c_{1,34}, c_{1,35}, c_{1,36}, c_{1,37}, c_{1,45}, c_{1,46}$, $c_{1,47}, c_{1,48}, c_{1,59}, c_{1,60}, c_{1,61}, c_{1,65}, c_{1,66}, c_{1,67}$ runs through the sets $I_{1}, I_{2}, \ldots$, $I_{16}$ respectively with $\left|I_{1}\right|=\left|I_{2}\right|=\left|I_{7}\right|=q^{2}-2,\left|I_{3}\right|=\left|I_{4}\right|=\left|I_{5}\right|=\left|I_{6}\right|=$ $\left|I_{9}\right|=\left|I_{11}\right|=\left|I_{14}\right|=\frac{q^{2}}{2}-1,\left|I_{8}\right|=\frac{q^{2}-2}{6},\left|I_{10}\right|=\frac{q^{2}+1}{3},\left|I_{12}\right|=\left|I_{16}\right|=\frac{q^{2}+\sqrt{2} q}{4}$, $\left|I_{13}\right|=\left|I_{15}\right|=\frac{q^{2}-\sqrt{2} q}{4}$. The sets $I_{1}, \ldots, I_{16}$ are defined in Section 4.)

\begin{tabular}{llll}
\hline Notation & Representative & $\left|C_{B}\right|$ & Fusion in $G$ \\
\hline$c_{1,0}$ & 1 & $q^{24}\left(q^{2}-1\right)^{2}$ & $c_{1,0}$ \\
$c_{1,1}$ & $\alpha_{12}(1)$ & $q^{24}\left(q^{2}-1\right)$ & $c_{1,1}$ \\
$c_{1,2}$ & $\alpha_{11}(1)$ & $q^{22}\left(q^{2}-1\right)$ & $c_{1,1}$ \\
$c_{1,3}$ & $\alpha_{10}(1)$ & $q^{20}\left(q^{2}-1\right)$ & $c_{1,2}$ \\
$c_{1,4}$ & $\alpha_{9}(1)$ & $q^{18}\left(q^{2}-1\right)$ & $c_{1,2}$ \\
$c_{1,5}$ & $\alpha_{8}(1)$ & $q^{18}\left(q^{2}-1\right)$ & $c_{1,1}$ \\
$c_{1,6}$ & $\alpha_{8}(1) \alpha_{11}(1)$ & $q^{18}$ & $c_{1,2}$ \\
\hline
\end{tabular}


Character table of a Borel subgroup of the Ree groups ${ }^{2} F_{4}\left(q^{2}\right)$

Table A.4 (continued)

\begin{tabular}{|c|c|c|c|}
\hline Notation & Representative & $\left|C_{B}\right|$ & Fusion in $G$ \\
\hline$c_{1,7}$ & $\alpha_{7}(1)$ & $q^{16}\left(q^{2}-1\right)$ & $c_{1,2}$ \\
\hline$c_{1,8}$ & $\alpha_{7}(1) \alpha_{8}(1)$ & $q^{16}$ & $c_{1,5}$ \\
\hline$c_{1,9}$ & $\alpha_{6}(1)$ & $2 q^{14}\left(q^{2}-1\right)$ & $c_{1,4}$ \\
\hline$c_{1,10}$ & $\alpha_{6}(1) \alpha_{11}(1)$ & $2 q^{14}\left(q^{2}-1\right)$ & $c_{1,3}$ \\
\hline$c_{1,11}$ & $\alpha_{6}(1) \alpha_{9}(1)$ & $q^{14}$ & $c_{1,6}$ \\
\hline$c_{1,12}$ & $\alpha_{5}(1)$ & $2 q^{14}\left(q^{2}-1\right)$ & $c_{1,4}$ \\
\hline$c_{1,13}$ & $\alpha_{5}(1) \alpha_{12}(1)$ & $2 q^{14}\left(q^{2}-1\right)$ & $c_{1,3}$ \\
\hline$c_{1,14}$ & $\alpha_{5}(1) \alpha_{7}(1)$ & $q^{14}$ & $c_{1,6}$ \\
\hline$c_{1,15}$ & $\alpha_{5}(1) \alpha_{6}(1)$ & $2 q^{12}$ & $c_{1,7}$ \\
\hline$c_{1,16}$ & $\alpha_{5}(1) \alpha_{6}(1) \alpha_{8}(1)$ & $2 q^{12}$ & $c_{1,8}$ \\
\hline$c_{1,17}$ & $\alpha_{4}(1)$ & $2 q^{12}\left(q^{2}-1\right)$ & $c_{1,4}$ \\
\hline$c_{1,18}$ & $\alpha_{4}(1) \alpha_{11}(1)$ & $2 q^{12}$ & $c_{1,6}$ \\
\hline$c_{1,19}$ & $\alpha_{4}(1) \alpha_{8}(1)$ & $2 q^{12}\left(q^{2}-1\right)$ & $c_{1,3}$ \\
\hline$c_{1,20}$ & $\alpha_{4}(1) \alpha_{8}(1) \alpha_{11}(1)$ & $2 q^{12}$ & $c_{1,6}$ \\
\hline$c_{1,21}$ & $\alpha_{4}(1) \alpha_{6}(1)$ & $2 q^{10}$ & $c_{1,7}$ \\
\hline$c_{1,22}$ & $\alpha_{4}(1) \alpha_{6}(1) \alpha_{11}(1)$ & $2 q^{10}$ & $c_{1,8}$ \\
\hline$c_{1,23}$ & $\alpha_{3}(1)$ & $q^{12}\left(q^{2}-1\right)$ & $c_{1,2}$ \\
\hline$c_{1,24}$ & $\alpha_{3}(1) \alpha_{11}(1)$ & $q^{12}$ & $c_{1,5}$ \\
\hline$c_{1,25}$ & $\alpha_{3}(1) \alpha_{6}(1)$ & $2 q^{12}$ & $c_{1,6}$ \\
\hline$c_{1,26}$ & $\alpha_{3}(1) \alpha_{6}(1) \alpha_{11}(1)$ & $2 q^{12}$ & $c_{1,6}$ \\
\hline$c_{1,27}$ & $\alpha_{3}(1) \alpha_{6}(1) \alpha_{9}(1)$ & $2 q^{12}$ & $c_{1,4}$ \\
\hline$c_{1,28}$ & $\alpha_{3}(1) \alpha_{6}(1) \alpha_{9}(1) \alpha_{11}(1)$ & $2 q^{12}$ & $c_{1,3}$ \\
\hline$c_{1,29}(a)$ & $\alpha_{3}(1) \alpha_{6}(1) \alpha_{9}(a)$ & $2 q^{12}$ & $c_{1,6}$ \\
\hline$c_{1,30}(a)$ & $\alpha_{3}(1) \alpha_{6}(1) \alpha_{9}(a) \alpha_{11}(1)$ & $2 q^{12}$ & $c_{1,6}$ \\
\hline$c_{1,31}$ & $\alpha_{3}(1) \alpha_{5}(1)$ & $q^{10}$ & $c_{1,6}$ \\
\hline$c_{1,32}$ & $\alpha_{3}(1) \alpha_{5}(1) \alpha_{6}(1)$ & $2 q^{10}$ & $c_{1,8}$ \\
\hline$c_{1,33}$ & $\alpha_{3}(1) \alpha_{5}(1) \alpha_{6}(1) \alpha_{8}(1)$ & $2 q^{10}$ & $c_{1,7}$ \\
\hline$c_{1,34}(a)$ & $\alpha_{3}(1) \alpha_{5}(a) \alpha_{6}(1)$ & $2 q^{10}$ & $c_{1,7}$ \\
\hline$c_{1,35}(a)$ & $\alpha_{3}(1) \alpha_{5}(a) \alpha_{6}(1)$ & $2 q^{10}$ & $c_{1,8}$ \\
\hline$c_{1,36}(a)$ & $\alpha_{3}(1) \alpha_{5}(a) \alpha_{6}(1) \alpha_{8}\left(t_{a}\right)$ & $2 q^{10}$ & $c_{1,7}$ \\
\hline$c_{1,37}(a)$ & $\alpha_{3}(1) \alpha_{5}(a) \alpha_{6}(1) \alpha_{8}\left(t_{a}\right)$ & $2 q^{10}$ & $c_{1,8}$ \\
\hline$c_{1,38}$ & $\alpha_{2}(1)$ & $q^{16}\left(q^{2}-1\right)$ & $c_{1,1}$ \\
\hline$c_{1,39}$ & $\alpha_{2}(1) \alpha_{12}(1)$ & $q^{16}$ & $c_{1,2}$ \\
\hline$c_{1,40}$ & $\alpha_{2}(1) \alpha_{9}(1)$ & $q^{14}$ & $c_{1,5}$ \\
\hline$c_{1,41}$ & $\alpha_{2}(1) \alpha_{8}(1)$ & $q^{12}$ & $c_{1,8}$ \\
\hline$c_{1,42}$ & $\alpha_{2}(1) \alpha_{6}(1)$ & $2 q^{14}$ & $c_{1,4}$ \\
\hline$c_{1,43}$ & $\alpha_{2}(1) \alpha_{6}(1) \alpha_{11}(1)$ & $2 q^{14}$ & $c_{1,3}$ \\
\hline$c_{1,44}$ & $\alpha_{2}(1) \alpha_{6}(1) \alpha_{9}(1)$ & $q^{14}$ & $c_{1,6}$ \\
\hline$c_{1,45}(a)$ & $\alpha_{2}(1) \alpha_{6}(a) \alpha_{9}(1)$ & $q^{14}$ & $c_{1,6}$ \\
\hline$c_{1,46}(a)$ & $\alpha_{2}(1) \alpha_{6}(a) \alpha_{8}(1)$ & $q^{12}$ & $c_{1,7}$ \\
\hline$c_{1,47}(a)$ & $\alpha_{2}(1) \alpha_{6}(a) \alpha_{8}(1)$ & $q^{12}$ & $c_{1,8}$ \\
\hline$c_{1,48}(a)$ & $\alpha_{2}(1) \alpha_{6}(a) \alpha_{8}(1)$ & $q^{12}$ & $c_{1,9}$ \\
\hline$c_{1,49}$ & $\alpha_{2}(1) \alpha_{4}(1)$ & $2 q^{8}$ & $c_{1,10}$ \\
\hline$c_{1,50}$ & $\alpha_{2}(1) \alpha_{4}(1) \alpha_{5}(1)$ & $4 q^{8}$ & $c_{1,11}$ \\
\hline$c_{1,51}$ & $\alpha_{2}(1) \alpha_{4}(1) \alpha_{5}(1) \alpha_{8}(1)$ & $4 q^{8}$ & $c_{1,12}$ \\
\hline$c_{1,52}$ & $\alpha_{2}(1) \alpha_{3}(1)$ & $2 q^{6}$ & $c_{1,13}$ \\
\hline$c_{1,53}$ & $\alpha_{2}(1) \alpha_{3}(1) \alpha_{5}(1)$ & $2 q^{6}$ & $c_{1,14}$ \\
\hline
\end{tabular}


Character table of a Borel subgroup of the Ree groups ${ }^{2} F_{4}\left(q^{2}\right)$

Table A.4 (continued)

\begin{tabular}{|c|c|c|c|}
\hline Notation & Representative & $\left|C_{B}\right|$ & Fusion in $G$ \\
\hline$c_{1,54}$ & $\alpha_{1}(1)$ & $2 q^{10}\left(q^{2}-1\right)$ & $c_{1,4}$ \\
\hline$c_{1,55}$ & $\alpha_{1}(1) \alpha_{12}(1)$ & $2 q^{10}$ & $c_{1,6}$ \\
\hline$c_{1,56}$ & $\alpha_{1}(1) \alpha_{8}(1)$ & $2 q^{8}$ & $c_{1,10}$ \\
\hline$c_{1,57}$ & $\alpha_{1}(1) \alpha_{5}(1)$ & $4 q^{8}$ & $c_{1,7}$ \\
\hline$c_{1,58}$ & $\alpha_{1}(1) \alpha_{5}(1) \alpha_{12}(1)$ & $4 q^{8}$ & $c_{1,8}$ \\
\hline$c_{1,59}(a)$ & $\alpha_{1}(1) \alpha_{6}(1) \alpha_{8}(a)$ & $2 q^{8}$ & $c_{1,10}$ \\
\hline$c_{1,60}(a)$ & $\alpha_{1}(1) \alpha_{6}(1) \alpha_{8}(a)$ & $2 q^{8}$ & $c_{1,11}$ \\
\hline$c_{1,61}(a)$ & $\alpha_{1}(1) \alpha_{6}(1) \alpha_{8}(a)$ & $2 q^{8}$ & $c_{1,12}$ \\
\hline$c_{1,62}$ & $\alpha_{1}(1) \alpha_{2}(1)$ & $2 q^{10}\left(q^{2}-1\right)$ & $c_{1,3}$ \\
\hline$c_{1,63}$ & $\alpha_{1}(1) \alpha_{2}(1) \alpha_{12}(1)$ & $2 q^{10}$ & $c_{1,6}$ \\
\hline$c_{1,64}$ & $\alpha_{1}(1) \alpha_{2}(1) \alpha_{8}(1)$ & $2 q^{8}$ & $c_{1,10}$ \\
\hline$c_{1,65}(a)$ & $\alpha_{1}(1) \alpha_{2}(1) \alpha_{6}(1) \alpha_{8}(a)$ & $2 q^{8}$ & $c_{1,10}$ \\
\hline$c_{1,66}(a)$ & $\alpha_{1}(1) \alpha_{2}(1) \alpha_{6}(1) \alpha_{8}(a)$ & $2 q^{8}$ & $c_{1,11}$ \\
\hline$c_{1,67}(a)$ & $\alpha_{1}(1) \alpha_{2}(1) \alpha_{6}(1) \alpha_{8}(a)$ & $2 q^{8}$ & $c_{1,12}$ \\
\hline$c_{1,68}$ & $\alpha_{1}(1) \alpha_{2}(1) \alpha_{6}(1)$ & $4 q^{8}$ & $c_{1,7}$ \\
\hline$c_{1,69}$ & $\alpha_{1}(1) \alpha_{2}(1) \alpha_{4}(1)$ & $4 q^{8}$ & $c_{1,8}$ \\
\hline$c_{1,70}$ & $\alpha_{1}(1) \alpha_{3}(1)$ & $4 q^{4}$ & $c_{1,15}$ \\
\hline$c_{1,71}$ & $\alpha_{1}(1) \alpha_{3}(1) \alpha_{5}(1)$ & $4 q^{4}$ & $c_{1,17}$ \\
\hline$c_{1,72}$ & $\alpha_{1}(1) \alpha_{2}(1) \alpha_{3}(1)$ & $4 q^{4}$ & $c_{1,16}$ \\
\hline$c_{1,73}$ & $\alpha_{1}(1) \alpha_{2}(1) \alpha_{3}(1) \alpha_{5}(1)$ & $4 q^{4}$ & $c_{1,18}$ \\
\hline$c_{2,0}(i)$ & $h_{2}(i)$ & $q^{4}\left(q^{2}-1\right)^{2}$ & $c_{2,0}(i)$ \\
\hline$c_{2,1}(i)$ & $h_{2}(i) \alpha_{2}(1)$ & $q^{4}\left(q^{2}-1\right)$ & $c_{2,1}(i)$ \\
\hline$c_{2,2}(i)$ & $h_{2}(i) \alpha_{1}(1)$ & $2 q^{2}\left(q^{2}-1\right)$ & $c_{2,2}(i)$ \\
\hline$c_{2,3}(i)$ & $h_{2}(i) \alpha_{1}(1) \alpha_{2}(1)$ & $2 q^{2}\left(q^{2}-1\right)$ & $c_{2,3}(i)$ \\
\hline$c_{3,0}(i)$ & $h_{3}(i)$ & $q^{2}\left(q^{2}-1\right)^{2}$ & $c_{3,0}(i)$ \\
\hline$c_{3,1}(i)$ & $h_{3}(i) \alpha_{3}(1)$ & $q^{2}\left(q^{2}-1\right)$ & $c_{3,1}(i)$ \\
\hline$c_{4,0}(i)$ & $h_{4}(i)$ & $q^{4}\left(q^{2}-1\right)^{2}$ & $c_{2,0}(2 \theta i)$ \\
\hline$c_{4,1}(i)$ & $h_{4}(i) \alpha_{8}(1)$ & $q^{4}\left(q^{2}-1\right)$ & $c_{2,1}(2 \theta i)$ \\
\hline$c_{4,2}(i)$ & $h_{4}(i) \alpha_{4}(1)$ & $2 q^{2}\left(q^{2}-1\right)$ & $c_{2,2}(2 \theta i)$ \\
\hline$c_{4,3}(i)$ & $h_{4}(i) \alpha_{4}(1) \alpha_{8}(1)$ & $2 q^{2}\left(q^{2}-1\right)$ & $c_{2,3}(2 \theta i)$ \\
\hline$c_{5,0}(i)$ & $h_{5}(i)$ & $q^{4}\left(q^{2}-1\right)^{2}$ & $c_{2,0}(i)$ \\
\hline$c_{5,1}(i)$ & $h_{5}(i) \alpha_{12}(1)$ & $q^{4}\left(q^{2}-1\right)$ & $c_{2,1}(i)$ \\
\hline$c_{5,2}(i)$ & $h_{5}(i) \alpha_{5}(1)$ & $2 q^{2}\left(q^{2}-1\right)$ & $c_{2,2}(i)$ \\
\hline$c_{5,3}(i)$ & $h_{5}(i) \alpha_{5}(1) \alpha_{12}(1)$ & $2 q^{2}\left(q^{2}-1\right)$ & $c_{2,3}(i)$ \\
\hline$c_{6,0}(i)$ & $h_{6}(i)$ & $q^{4}\left(q^{2}-1\right)^{2}$ & $c_{2,0}(2 \theta i)$ \\
\hline$c_{6,1}(i)$ & $h_{6}(i) \alpha_{11}(1)$ & $q^{4}\left(q^{2}-1\right)$ & $c_{2,1}(2 \theta i)$ \\
\hline$c_{6,2}(i)$ & $h_{6}(i) \alpha_{6}(1)$ & $2 q^{2}\left(q^{2}-1\right)$ & $c_{2,2}(2 \theta i)$ \\
\hline$c_{6,3}(i)$ & $h_{6}(i) \alpha_{6}(1) \alpha_{11}(1)$ & $2 q^{2}\left(q^{2}-1\right)$ & $c_{2,3}(2 \theta i)$ \\
\hline$c_{7,0}(i)$ & $h_{7}(i)$ & $q^{2}\left(q^{2}-1\right)^{2}$ & $c_{3,0}(i)$ \\
\hline$c_{7,1}(i)$ & $h_{7}(i) \alpha_{7}(1)$ & $q^{2}\left(q^{2}-1\right)$ & $c_{3,1}(i)$ \\
\hline$c_{8,0}(i)$ & $h_{8}(i)$ & $q^{2}\left(q^{2}-1\right)^{2}$ & $c_{3,0}(i)$ \\
\hline$c_{8,1}(i)$ & $h_{8}(i) \alpha_{9}(1)$ & $q^{2}\left(q^{2}-1\right)$ & $c_{3,1}(i)$ \\
\hline$c_{9,0}(i)$ & $h_{9}(i)$ & $q^{2}\left(q^{2}-1\right)^{2}$ & $c_{3,0}(-i)$ \\
\hline$c_{9,1}(i)$ & $h_{9}(i) \alpha_{10}(1)$ & $q^{2}\left(q^{2}-1\right)$ & $c_{3,1}(-i)$ \\
\hline$c_{10,0}(i, j)$ & $h_{10}(i, j)$ & $\left(q^{2}-1\right)^{2}$ & $c_{4,0}(i, j)$ \\
\hline
\end{tabular}


Character table of a Borel subgroup of the Ree groups ${ }^{2} F_{4}\left(q^{2}\right)$

Table A.5: Parametrization of the irreducible characters of $B$.

\begin{tabular}{|c|c|c|c|}
\hline Character & Degree & Parameters & Number of Characters \\
\hline$B \chi_{1}(k, l)$ & 1 & $k, l=0, \ldots, q^{2}-2$ & $\left(q^{2}-1\right)^{2}$ \\
\hline${ }_{B} \chi_{2}(k)$ & $q^{2}-1$ & $k=0, \ldots, q^{2}-2$ & $q^{2}-1$ \\
\hline${ }_{B} \chi_{3}(k)$ & $\frac{q}{\sqrt{2}}\left(q^{2}-1\right)$ & $k=0, \ldots, q^{2}-2$ & $q^{2}-1$ \\
\hline${ }_{B} \chi_{4}(k)$ & $\frac{q}{\sqrt{2}}\left(q^{2}-1\right)$ & $k=0, \ldots, q^{2}-2$ & $q^{2}-1$ \\
\hline$B \chi_{5}(k)$ & $q^{2}-1$ & $k=0, \ldots, q^{2}-2$ & $q^{2}-1$ \\
\hline$B \chi_{6}$ & $\frac{q}{\sqrt{2}}\left(q^{2}-1\right)^{2}$ & & 1 \\
\hline$B \chi_{7}$ & $\frac{q}{\sqrt{2}}\left(q^{2}-1\right)^{2}$ & & 1 \\
\hline$B \chi_{8}$ & $\left(q^{2}-1\right)^{2}$ & & 1 \\
\hline$B \chi_{9}(k)$ & $q^{2}\left(q^{2}-1\right)$ & $k=0, \ldots, q^{2}-2$ & $q^{2}-1$ \\
\hline$B \chi_{10}$ & $q^{2}\left(q^{2}-1\right)^{2}$ & & 1 \\
\hline$B \chi_{11}(k)$ & $q^{4}\left(q^{2}-1\right)$ & $k=0, \ldots, q^{2}-2$ & $q^{2}-1$ \\
\hline${ }_{B} \chi_{12}(k)$ & $q^{4}\left(q^{2}-1\right)$ & $k=0, \ldots, q^{2}-2$ & $q^{2}-1$ \\
\hline${ }_{B} \chi_{13}$ & $\frac{q^{4}}{2}\left(q^{2}-1\right)^{2}$ & & 1 \\
\hline$B \chi_{14}$ & $\frac{q^{4}}{2}\left(q^{2}-1\right)^{2}$ & & 1 \\
\hline$B \chi_{15}$ & $\frac{q^{4}}{4}\left(q^{2}-1\right)^{2}$ & & 1 \\
\hline$B \chi_{16}$ & $\frac{q^{4}}{4}\left(q^{2}-1\right)^{2}$ & & 1 \\
\hline$B \chi_{17}$ & $\frac{q^{4}}{4}\left(q^{2}-1\right)^{2}$ & & 1 \\
\hline${ }_{B} \chi_{18}$ & $\frac{q^{4}}{4}\left(q^{2}-1\right)^{2}$ & & 1 \\
\hline${ }_{B} \chi_{19}$ & $\frac{q^{4}}{4}\left(q^{2}-1\right)^{2}$ & & 1 \\
\hline$B \chi_{20}$ & $\frac{q^{4}}{4}\left(q^{2}-1\right)^{2}$ & & 1 \\
\hline${ }_{B} \chi_{21}$ & $\frac{q^{4}}{4}\left(q^{2}-1\right)^{2}$ & & 1 \\
\hline$B \chi_{22}$ & $\frac{q^{4}}{4}\left(q^{2}-1\right)^{2}$ & & 1 \\
\hline${ }_{B} \chi_{23}(k)$ & $q^{4}\left(q^{2}-1\right)$ & $k=0, \ldots, q^{2}-2$ & $q^{2}-1$ \\
\hline$B \chi_{24}$ & $q^{4}\left(q^{2}-1\right)^{2}$ & & 1 \\
\hline$B \chi_{25}(k)$ & $q^{4}\left(q^{2}-1\right)^{2}$ & $k=1, \ldots, q^{2}$ & $q^{2}$ \\
\hline${ }_{B} \chi_{26}(k)$ & $\frac{q^{3}}{\sqrt{2}}\left(q^{2}-1\right)$ & $k=0, \ldots, q^{2}-2$ & $q^{2}-1$ \\
\hline${ }_{B} \chi_{27}(k)$ & $\frac{q^{3}}{\sqrt{2}}\left(q^{2}-1\right)$ & $k=0, \ldots, q^{2}-2$ & $q^{2}-1$ \\
\hline$B \chi_{28}$ & $\frac{q^{3}}{\sqrt{2}}\left(q^{2}-1\right)^{2}$ & & 1 \\
\hline$B \chi_{29}$ & $\frac{q^{3}}{\sqrt{2}}\left(q^{2}-1\right)^{2}$ & & 1 \\
\hline$B \chi_{30}$ & $\frac{q^{4}}{2}\left(q^{2}-1\right)^{2}$ & & 1 \\
\hline$B \chi_{31}$ & $\frac{q^{4}}{2}\left(q^{2}-1\right)^{2}$ & & 1 \\
\hline
\end{tabular}


Character table of a Borel subgroup of the Ree groups ${ }^{2} F_{4}\left(q^{2}\right)$

Table A.5 (continued)

\begin{tabular}{|c|c|c|c|}
\hline Character & Degree & Parameters & Number of Characters \\
\hline$B \chi_{32}$ & $\frac{q^{4}}{2}\left(q^{2}-1\right)^{2}$ & & 1 \\
\hline$B \chi_{33}$ & $\frac{q^{4}}{2}\left(q^{2}-1\right)^{2}$ & & 1 \\
\hline$B \chi_{34}$ & $q^{4}\left(q^{2}-1\right)^{2}$ & & 1 \\
\hline${ }_{B} \chi_{35}(k)$ & $\frac{q^{4}}{2}\left(q^{2}-1\right)^{2}$ & $k=1, \ldots, 4 \cdot \frac{q^{2}-2}{6}$ & $4 \cdot \frac{q^{2}-2}{6}$ \\
\hline$B \chi_{36}(k)$ & $\frac{q^{4}}{2}\left(q^{2}-1\right)^{2}$ & $k=1, \ldots, 4 \cdot \frac{q^{2}-2}{2}$ & $4 \cdot \frac{q^{2}-2}{2}$ \\
\hline$B \chi_{37}(k)$ & $q^{4}\left(q^{2}-1\right)^{2}$ & $k=1, \ldots, \frac{q^{2}+1}{3}$ & $\frac{q^{2}+1}{3}$ \\
\hline$B \chi_{38}$ & $q^{6}\left(q^{2}-1\right)^{2}$ & & 1 \\
\hline${ }_{B} \chi_{39}(k)$ & $q^{6}\left(q^{2}-1\right)$ & $k=0, \ldots, q^{2}-2$ & $q^{2}-1$ \\
\hline$B \chi_{40}$ & $q^{6}\left(q^{2}-1\right)^{2}$ & & 1 \\
\hline${ }_{B} \chi_{41}(k)$ & $q^{6}\left(q^{2}-1\right)^{2}$ & $k=1, \ldots, q^{2}$ & $q^{2}$ \\
\hline${ }_{B} \chi_{42}(k)$ & $q^{8}\left(q^{2}-1\right)$ & $k=0, \ldots, q^{2}-2$ & $q^{2}-1$ \\
\hline$B \chi_{43}$ & $q^{8}\left(q^{2}-1\right)^{2}$ & & 1 \\
\hline${ }_{B} \chi_{44}(k)$ & $\frac{q^{7}}{\sqrt{2}}\left(q^{2}-1\right)$ & $k=0, \ldots, q^{2}-2$ & $q^{2}-1$ \\
\hline${ }_{B} \chi_{45}(k)$ & $\frac{q^{7}}{\sqrt{2}}\left(q^{2}-1\right)$ & $k=0, \ldots, q^{2}-2$ & $q^{2}-1$ \\
\hline$B \chi_{46}$ & $\frac{q^{7}}{\sqrt{2}}\left(q^{2}-1\right)^{2}$ & & 1 \\
\hline$B \chi_{47}$ & $\frac{q^{7}}{\sqrt{2}}\left(q^{2}-1\right)^{2}$ & & 1 \\
\hline${ }_{B} \chi_{48}(k)$ & $\frac{q^{7}}{\sqrt{2}}\left(q^{2}-1\right)^{2}$ & $k=1, \ldots, q^{2}$ & $q^{2}$ \\
\hline${ }_{B} \chi_{49}(k)$ & $\frac{q^{7}}{\sqrt{2}}\left(q^{2}-1\right)^{2}$ & $k=1, \ldots, q^{2}$ & $q^{2}$ \\
\hline${ }_{B} \chi_{50}(k)$ & $q^{8}\left(q^{2}-1\right)^{2}$ & $k=1, \ldots, q^{2}$ & $q^{2}$ \\
\hline$B \chi_{51}(k)$ & $\frac{q^{9}}{\sqrt{2}}\left(q^{2}-1\right)$ & $k=0, \ldots, q^{2}-2$ & $q^{2}-1$ \\
\hline${ }_{B} \chi_{52}(k)$ & $\frac{q^{9}}{\sqrt{2}}\left(q^{2}-1\right)$ & $k=0, \ldots, q^{2}-2$ & $q^{2}-1$ \\
\hline$B \chi_{53}$ & $\frac{q^{9}}{\sqrt{2}}\left(q^{2}-1\right)^{2}$ & & 1 \\
\hline$B \chi_{54}$ & $\frac{q^{9}}{\sqrt{2}}\left(q^{2}-1\right)^{2}$ & & 1 \\
\hline$B \chi_{55}$ & $\frac{q^{10}}{2}\left(q^{2}-1\right)^{2}$ & & 1 \\
\hline$B \chi_{56}$ & $\frac{q^{10}}{2}\left(q^{2}-1\right)^{2}$ & & 1 \\
\hline$B \chi_{57}$ & $\frac{q^{10}}{2}\left(q^{2}-1\right)^{2}$ & & 1 \\
\hline$B \chi_{58}$ & $\frac{q^{10}}{2}\left(q^{2}-1\right)^{2}$ & & 1 \\
\hline
\end{tabular}


Character table of a Borel subgroup of the Ree groups ${ }^{2} F_{4}\left(q^{2}\right)$

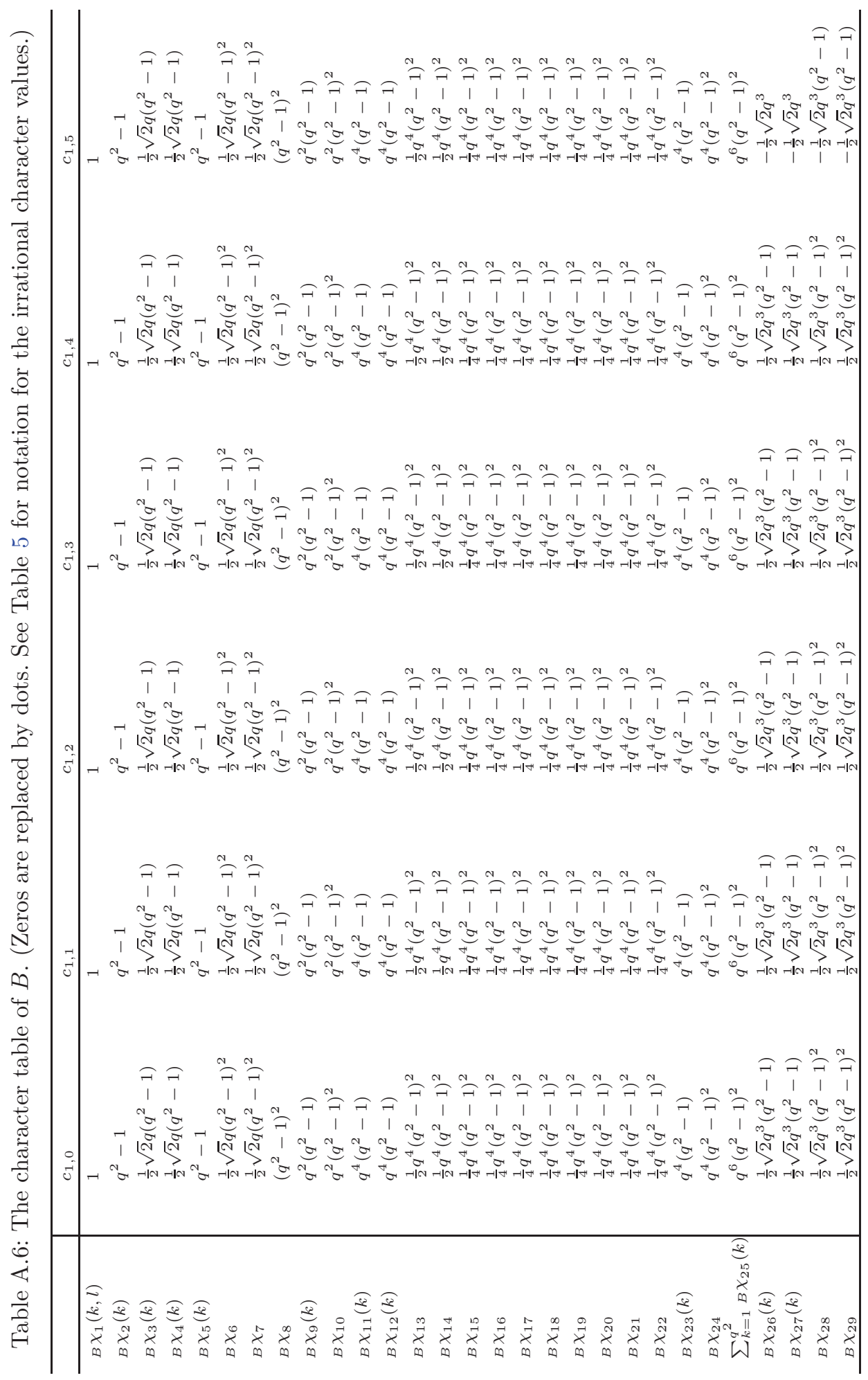


Character table of a Borel subgroup of the Ree groups ${ }^{2} F_{4}\left(q^{2}\right)$

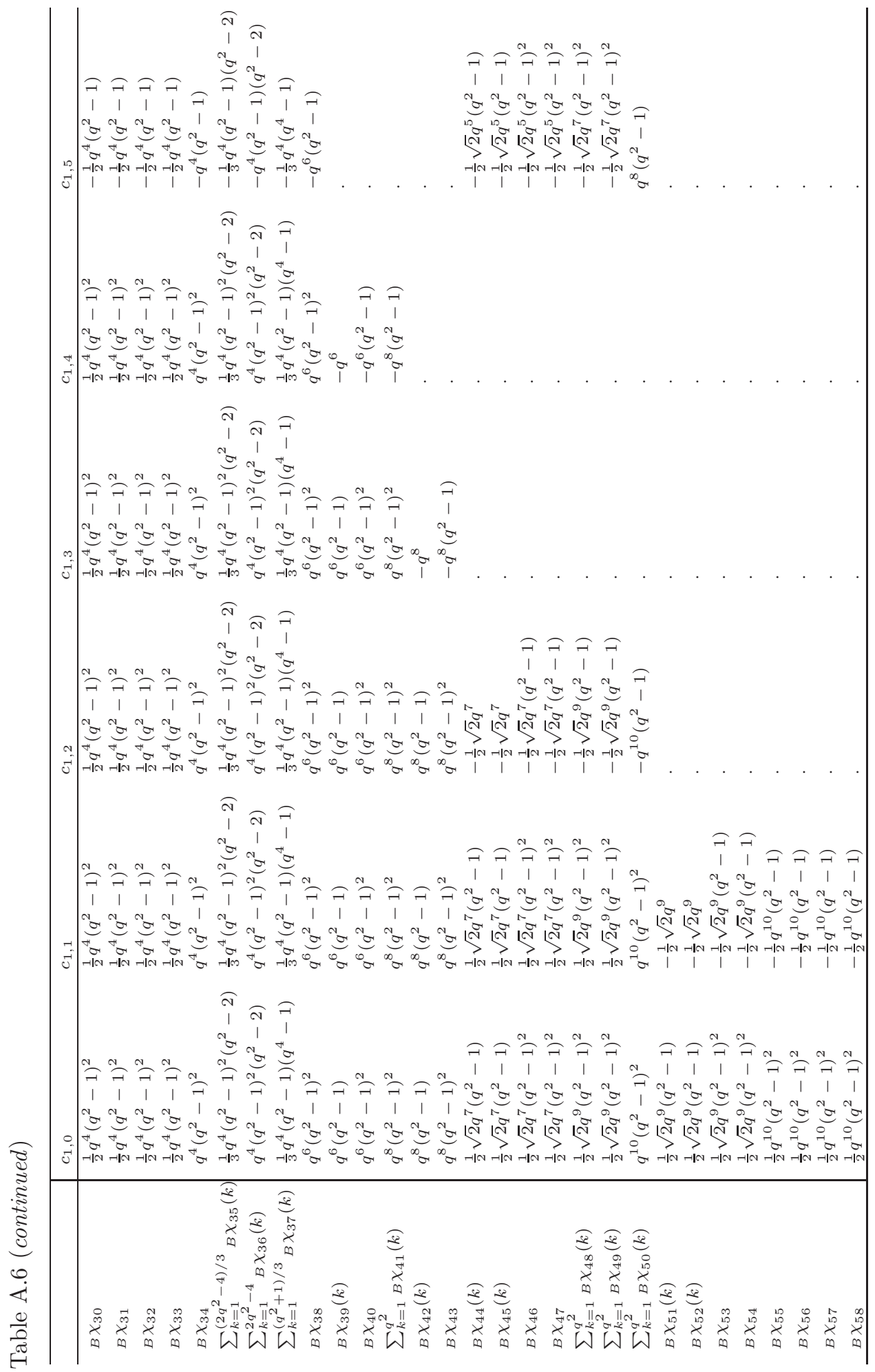


Character table of a Borel subgroup of the Ree groups ${ }^{2} F_{4}\left(q^{2}\right)$

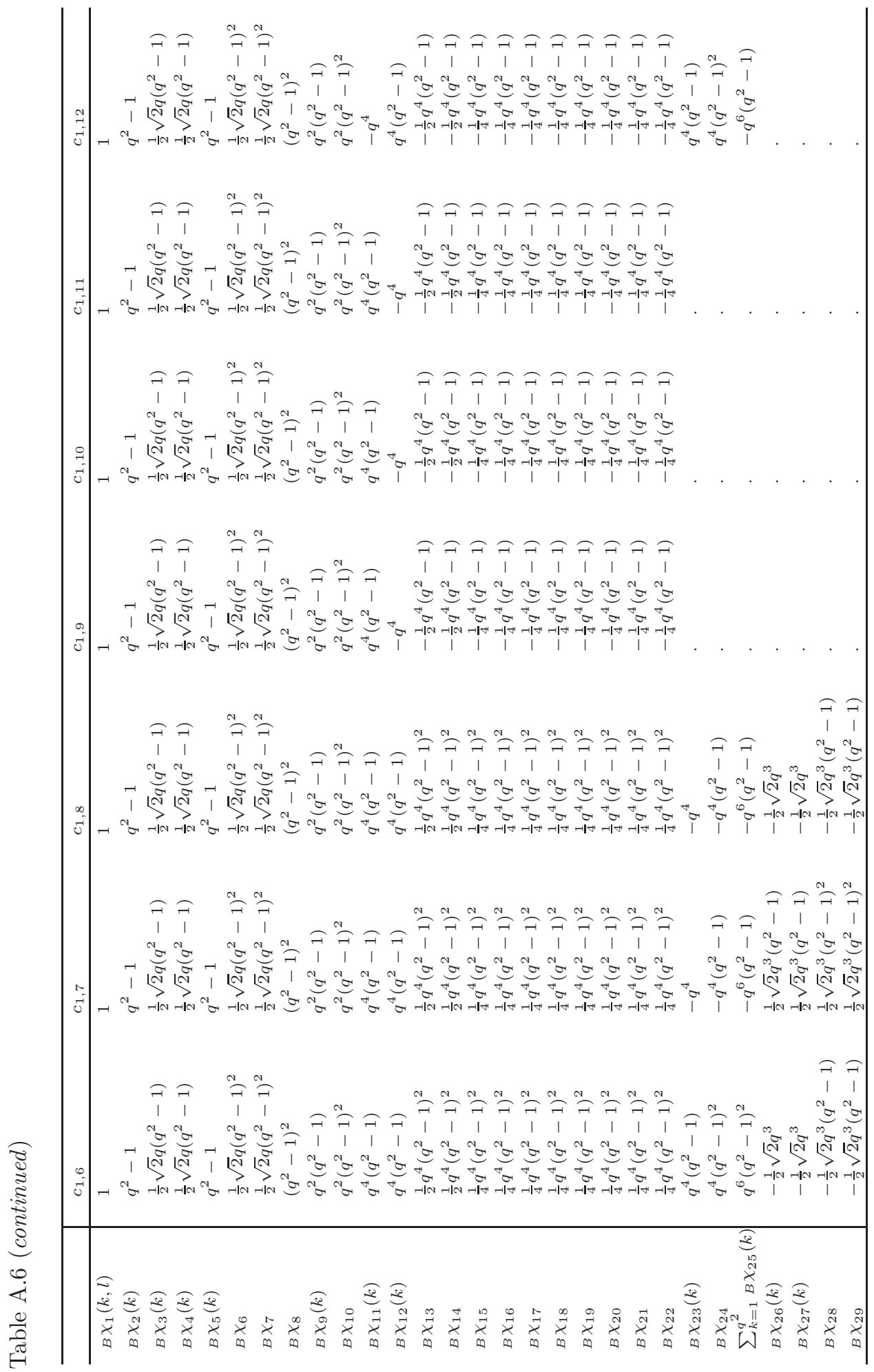


Character table of a Borel subgroup of the Ree groups ${ }^{2} F_{4}\left(q^{2}\right)$

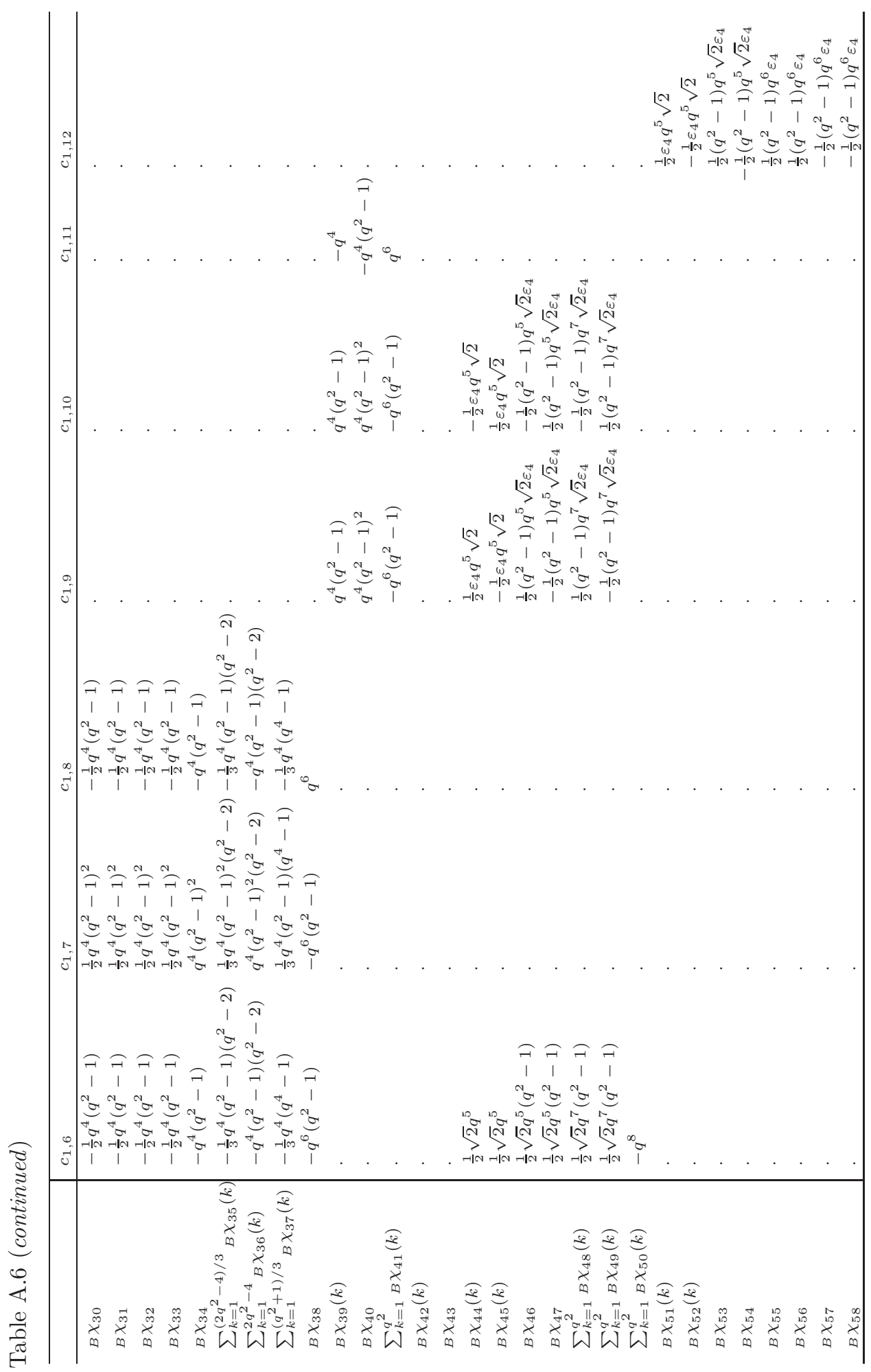


Character table of a Borel subgroup of the Ree groups ${ }^{2} F_{4}\left(q^{2}\right)$

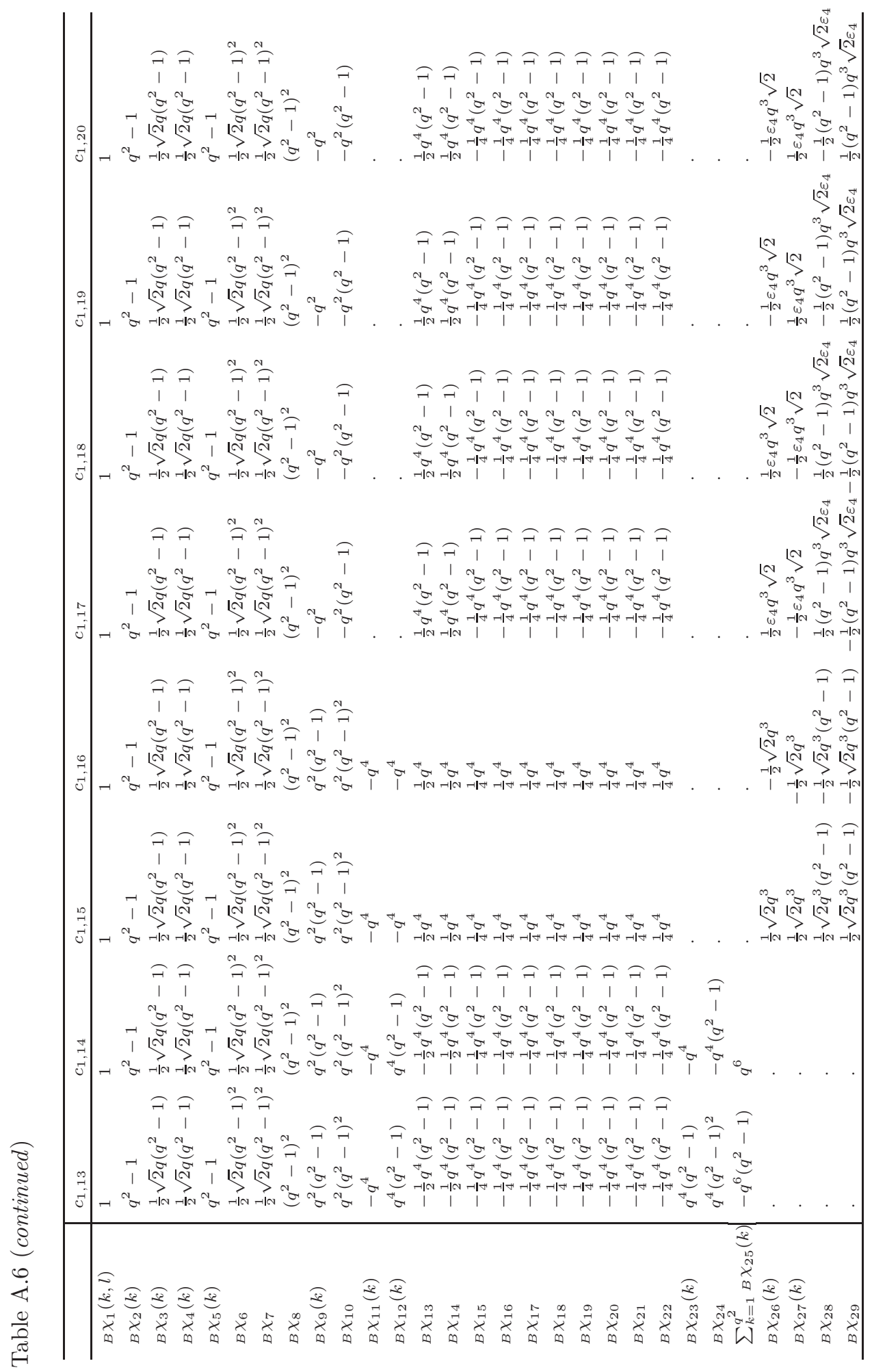


Character table of a Borel subgroup of the Ree groups ${ }^{2} F_{4}\left(q^{2}\right)$

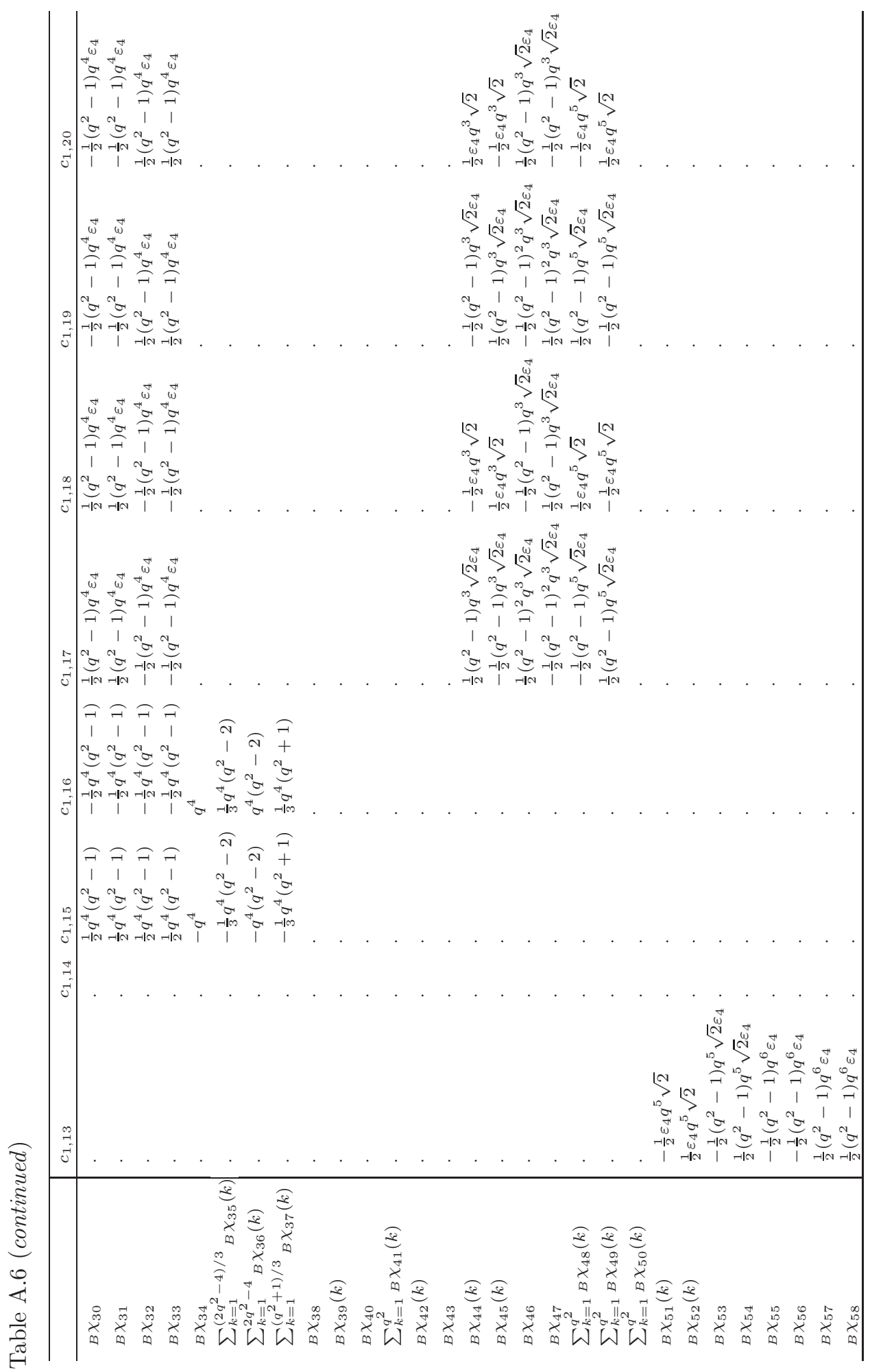


Character table of a Borel subgroup of the Ree groups ${ }^{2} F_{4}\left(q^{2}\right)$

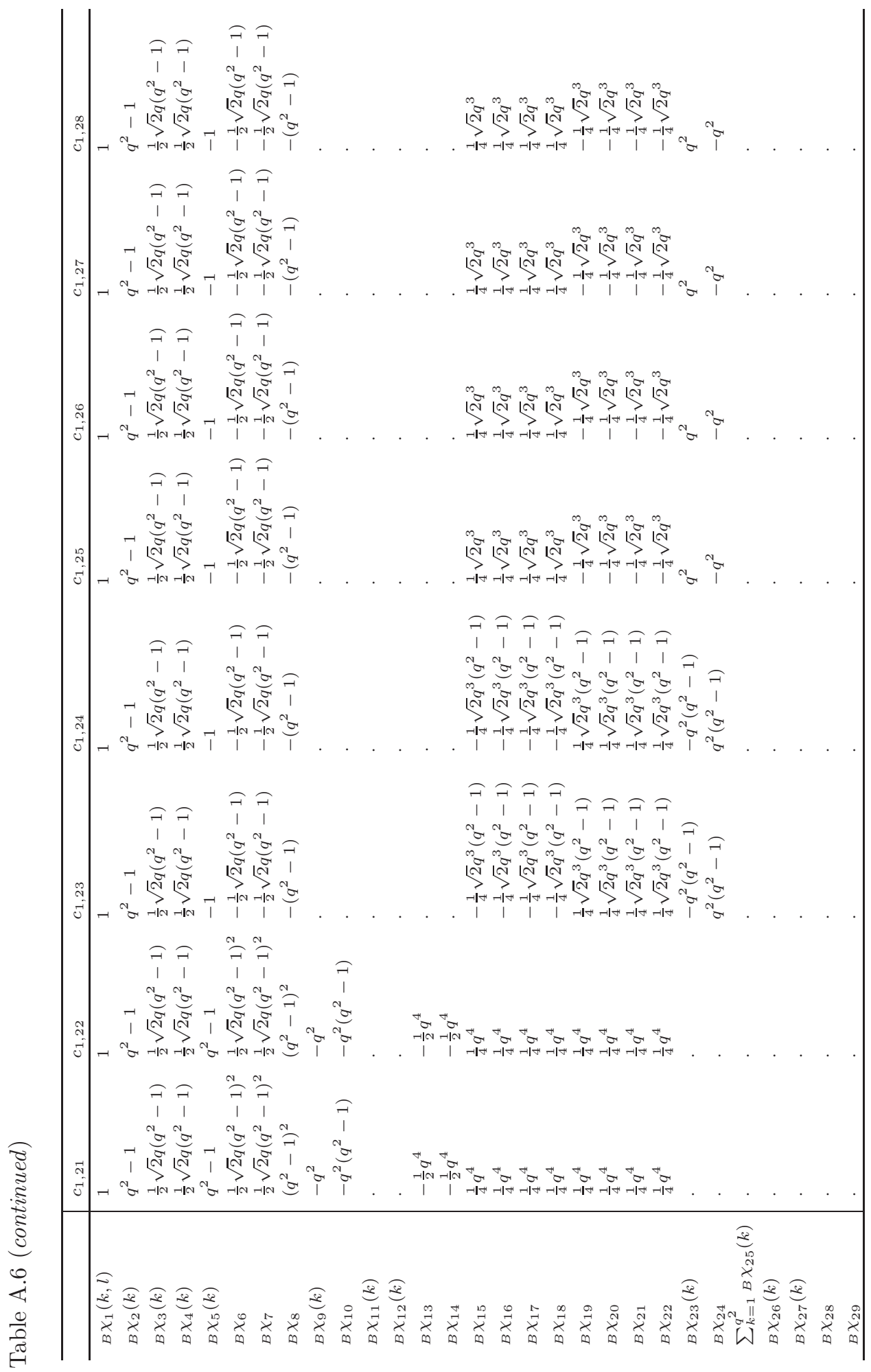


Character table of a Borel subgroup of the Ree groups ${ }^{2} F_{4}\left(q^{2}\right)$

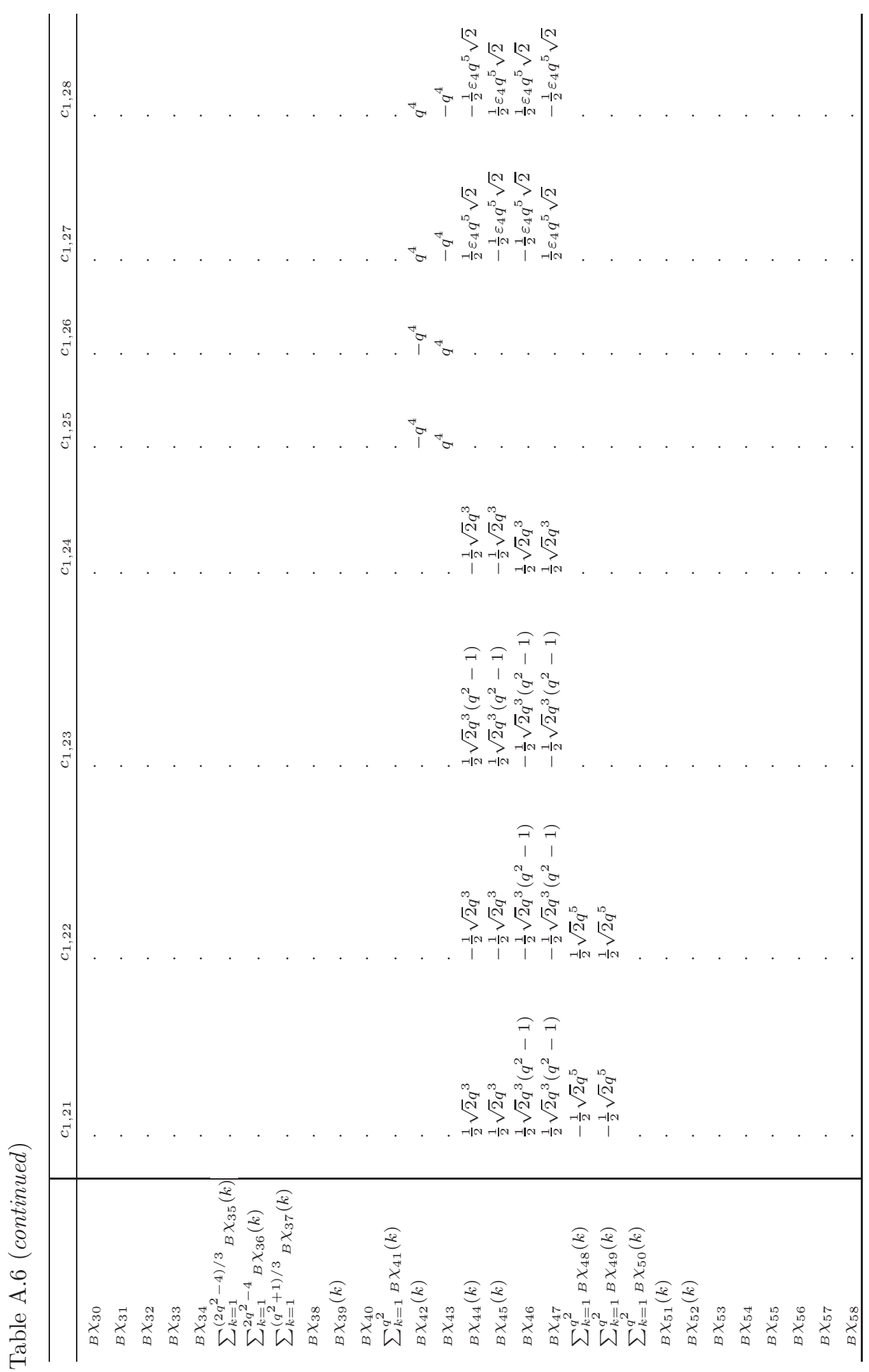


Character table of a Borel subgroup of the Ree groups ${ }^{2} F_{4}\left(q^{2}\right)$

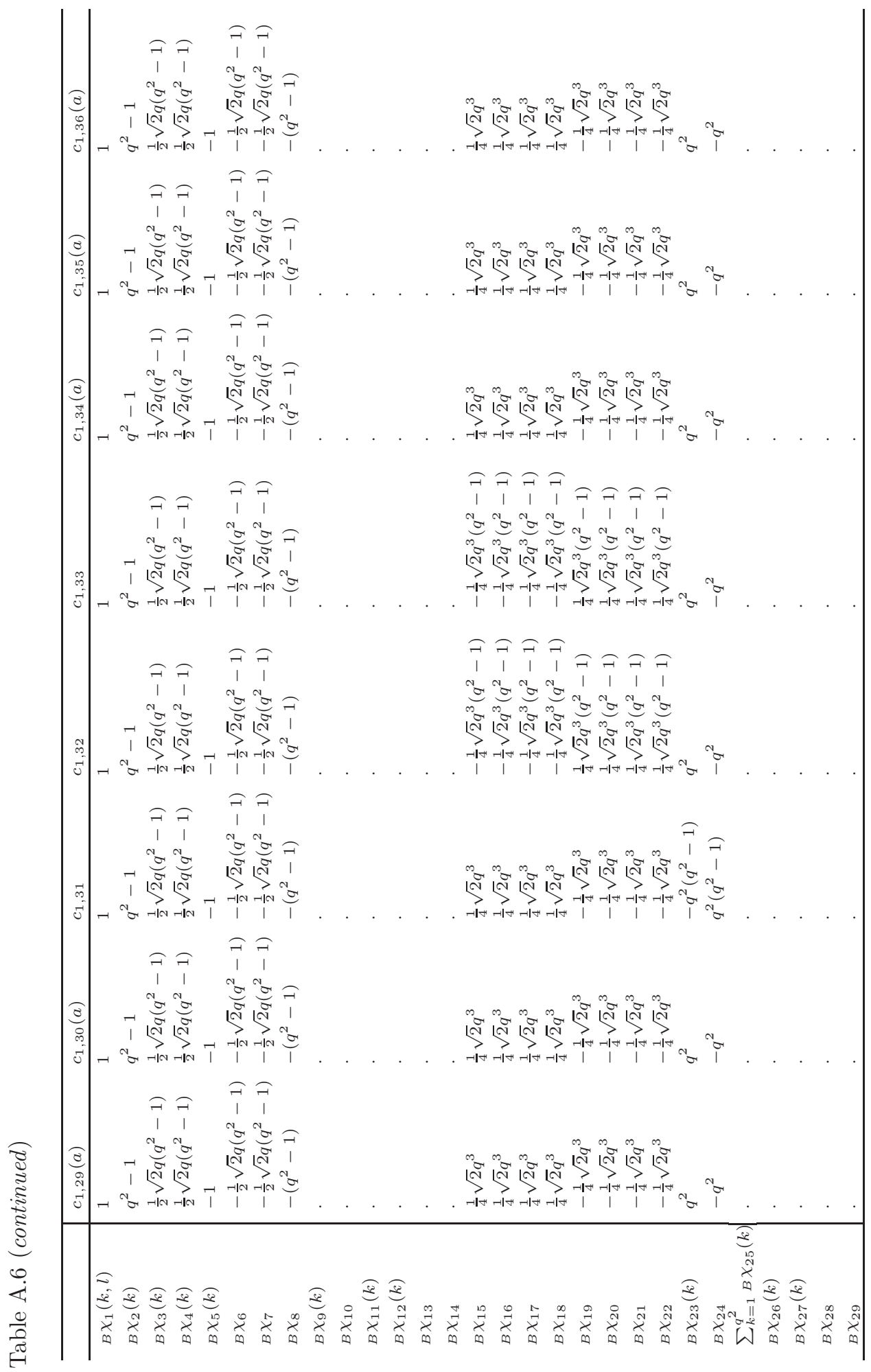


Character table of a Borel subgroup of the Ree groups ${ }^{2} F_{4}\left(q^{2}\right)$

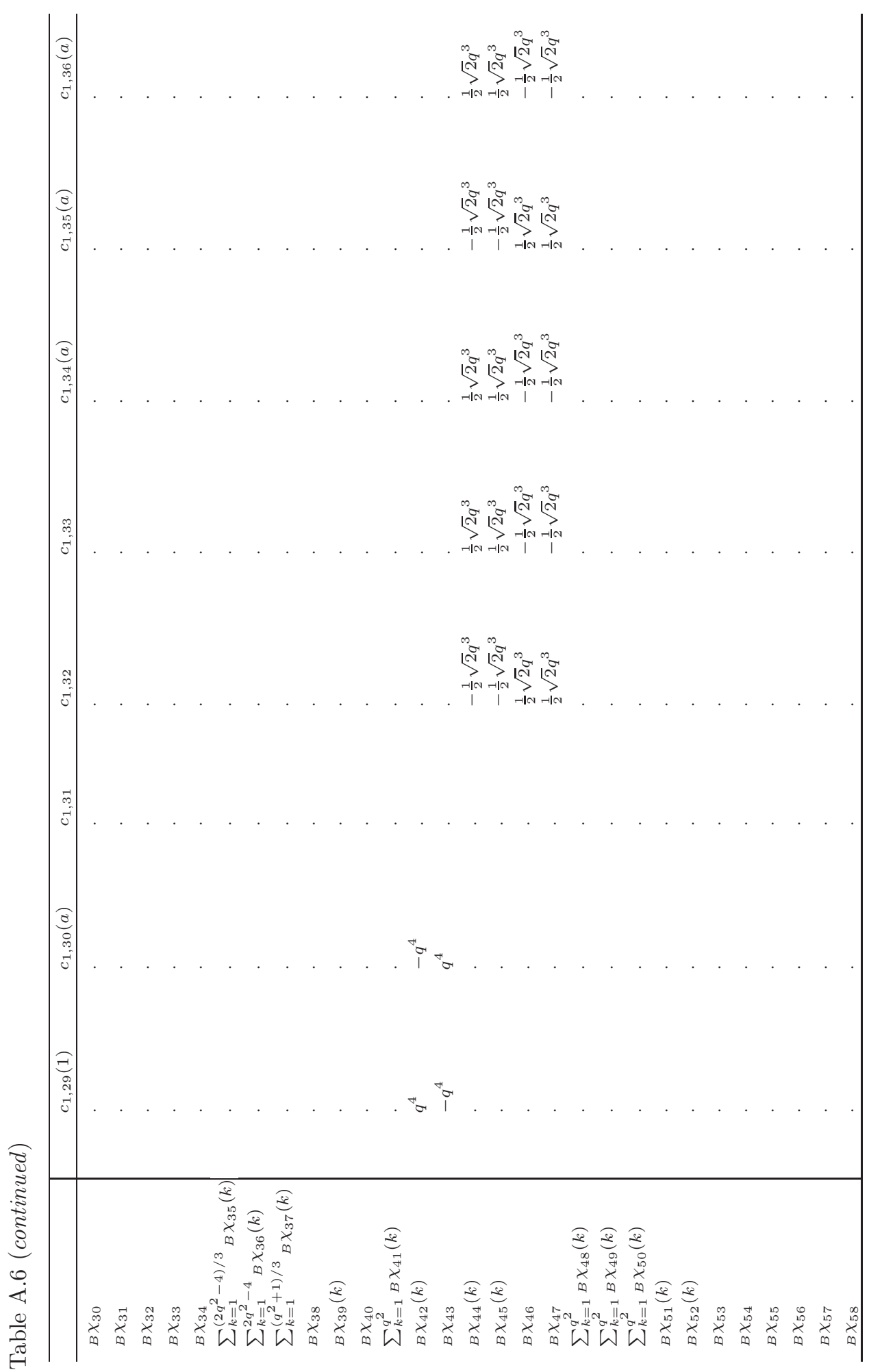


Character table of a Borel subgroup of the Ree groups ${ }^{2} F_{4}\left(q^{2}\right)$

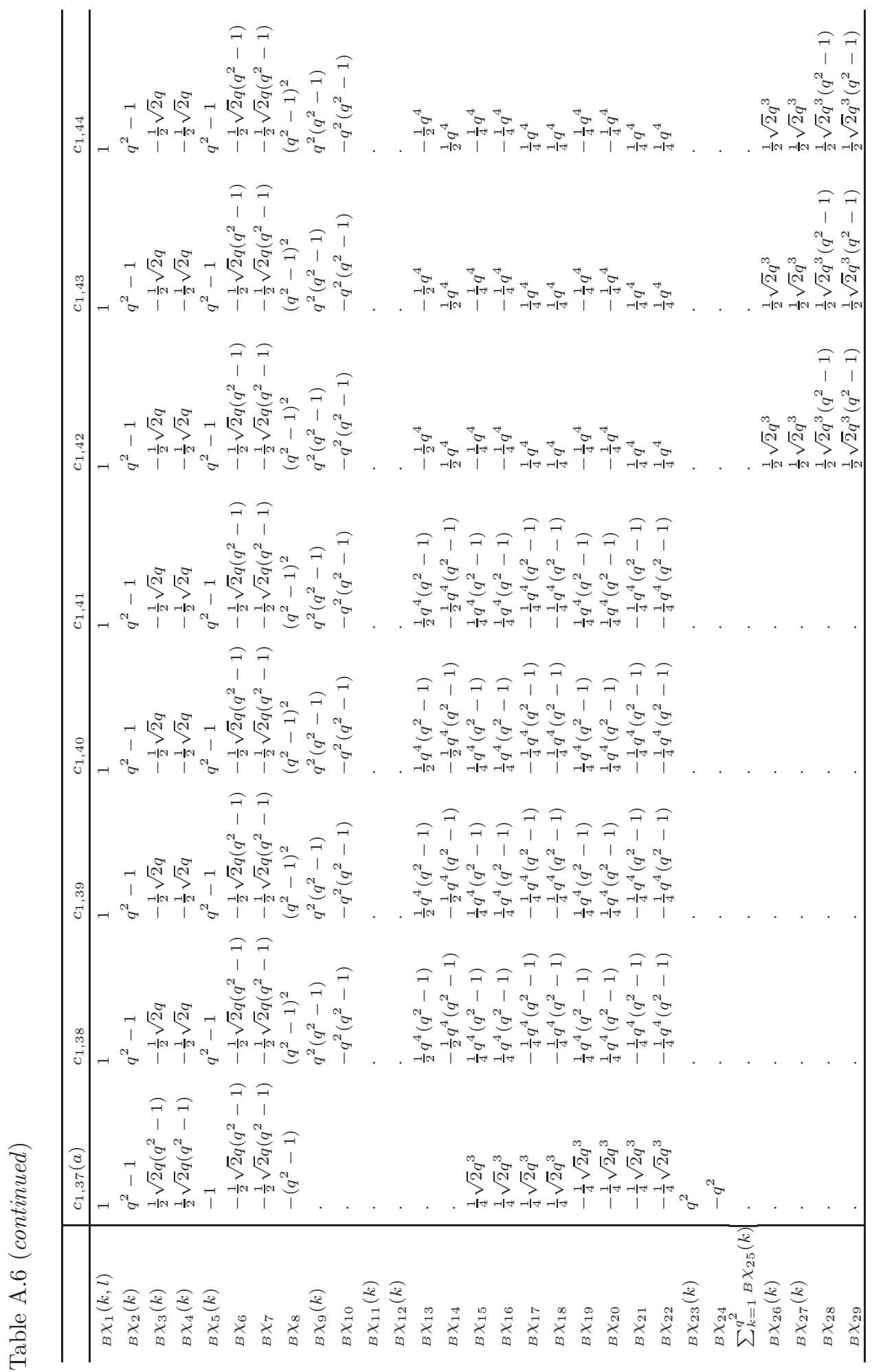


Character table of a Borel subgroup of the Ree groups ${ }^{2} F_{4}\left(q^{2}\right)$

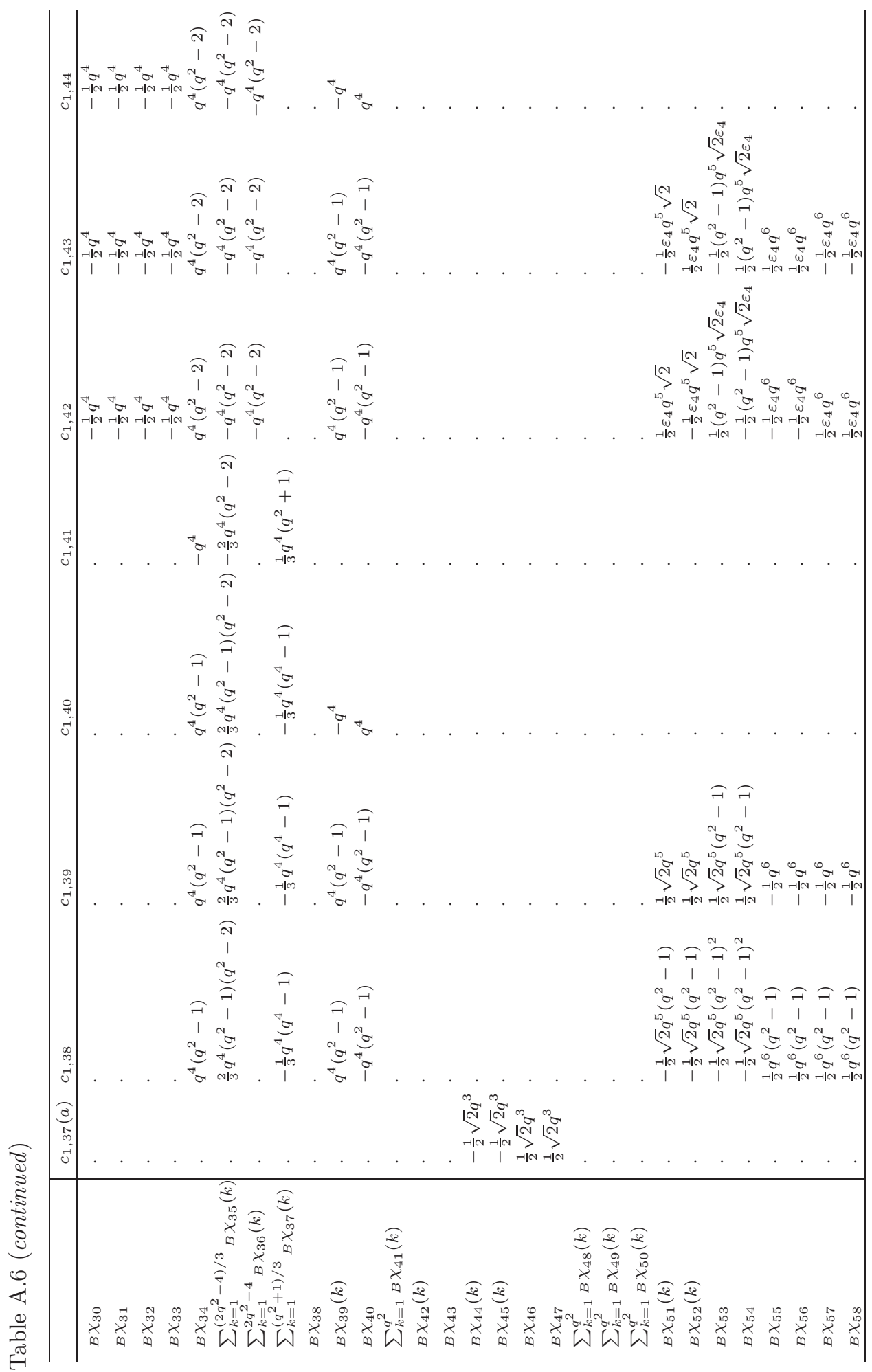


Character table of a Borel subgroup of the Ree groups ${ }^{2} F_{4}\left(q^{2}\right)$

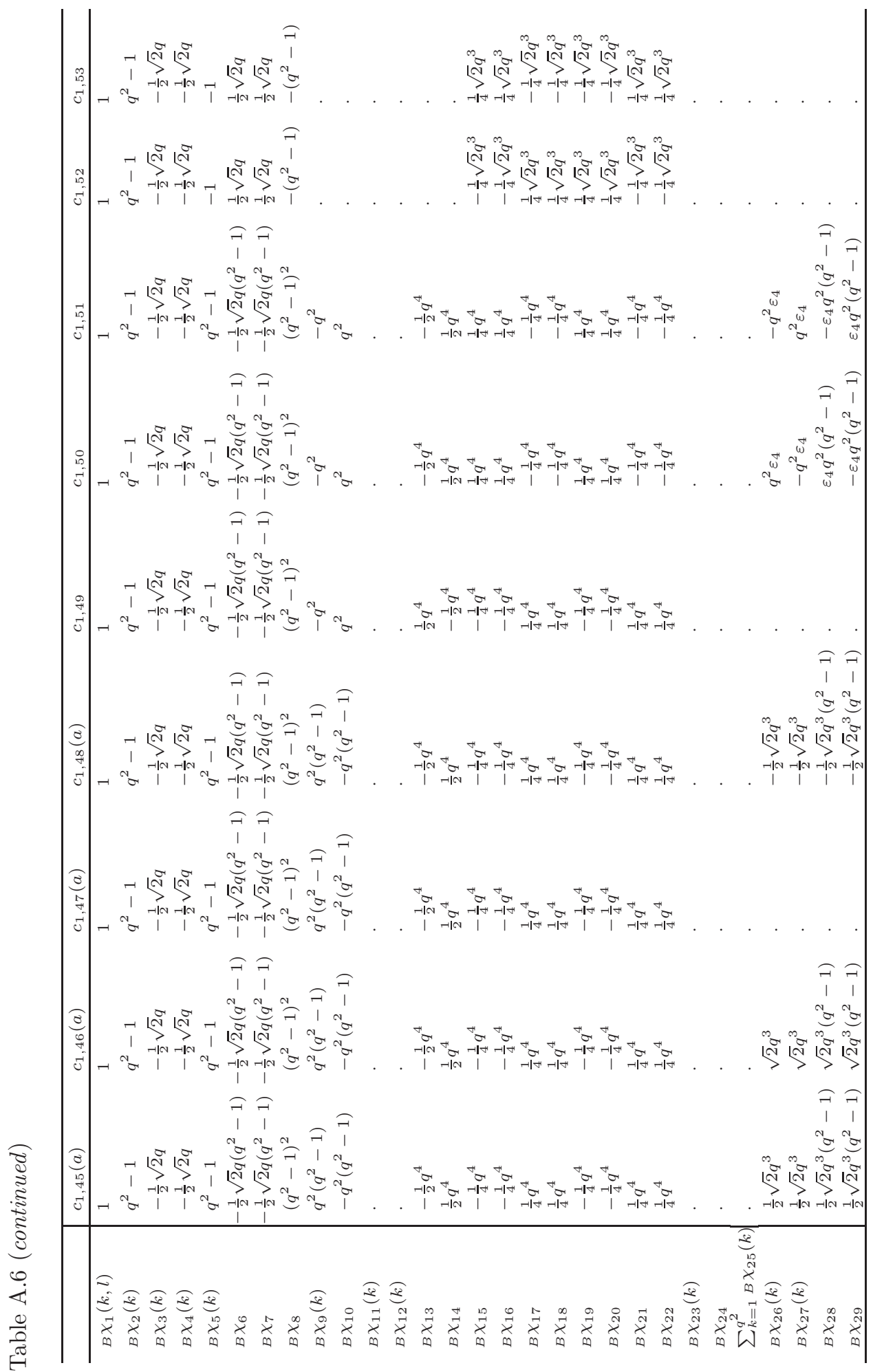


Character table of a Borel subgroup of the Ree groups ${ }^{2} F_{4}\left(q^{2}\right)$

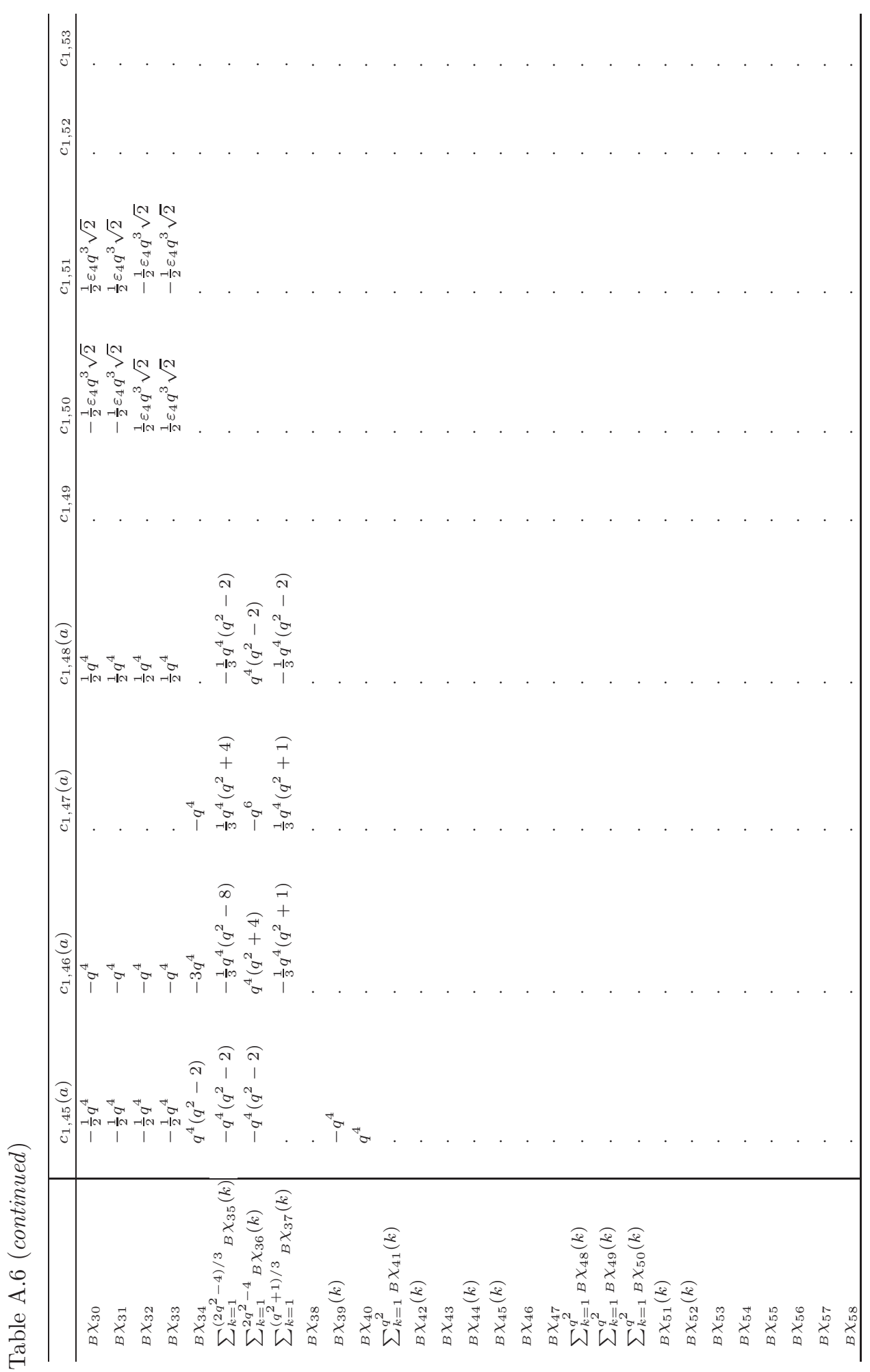


Character table of a Borel subgroup of the Ree groups ${ }^{2} F_{4}\left(q^{2}\right)$

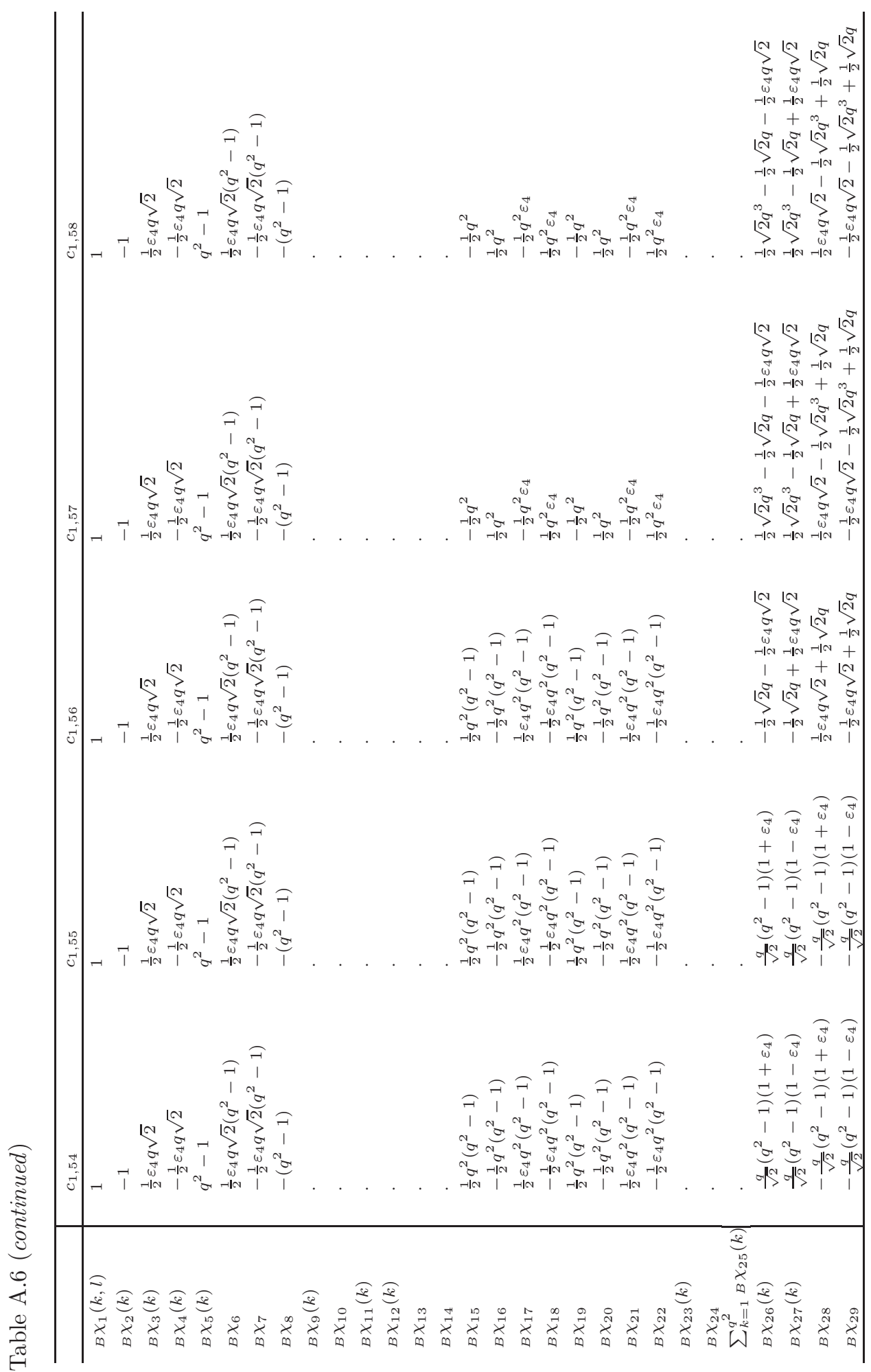


Character table of a Borel subgroup of the Ree groups ${ }^{2} F_{4}\left(q^{2}\right)$

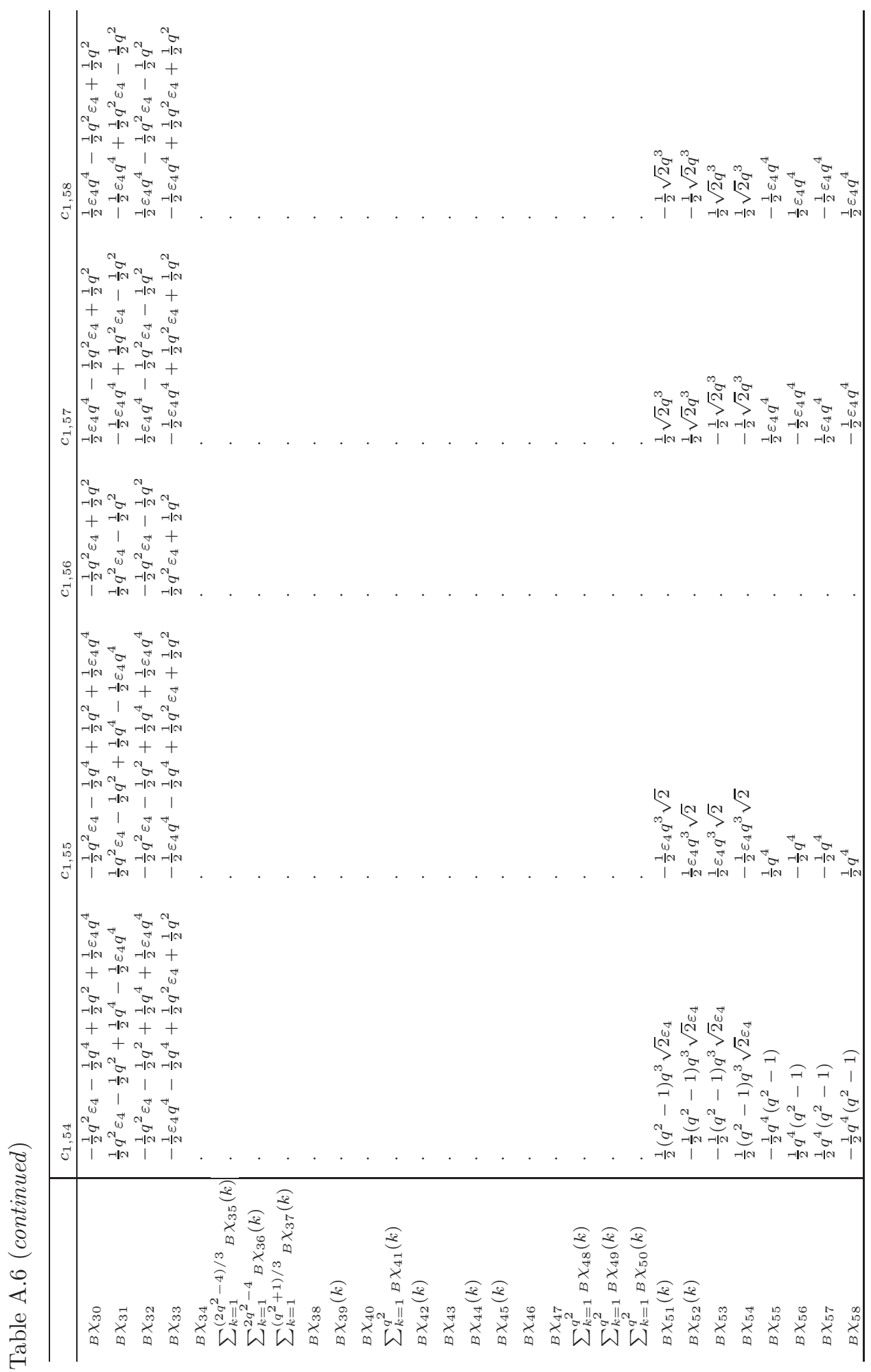


Character table of a Borel subgroup of the Ree groups ${ }^{2} F_{4}\left(q^{2}\right)$

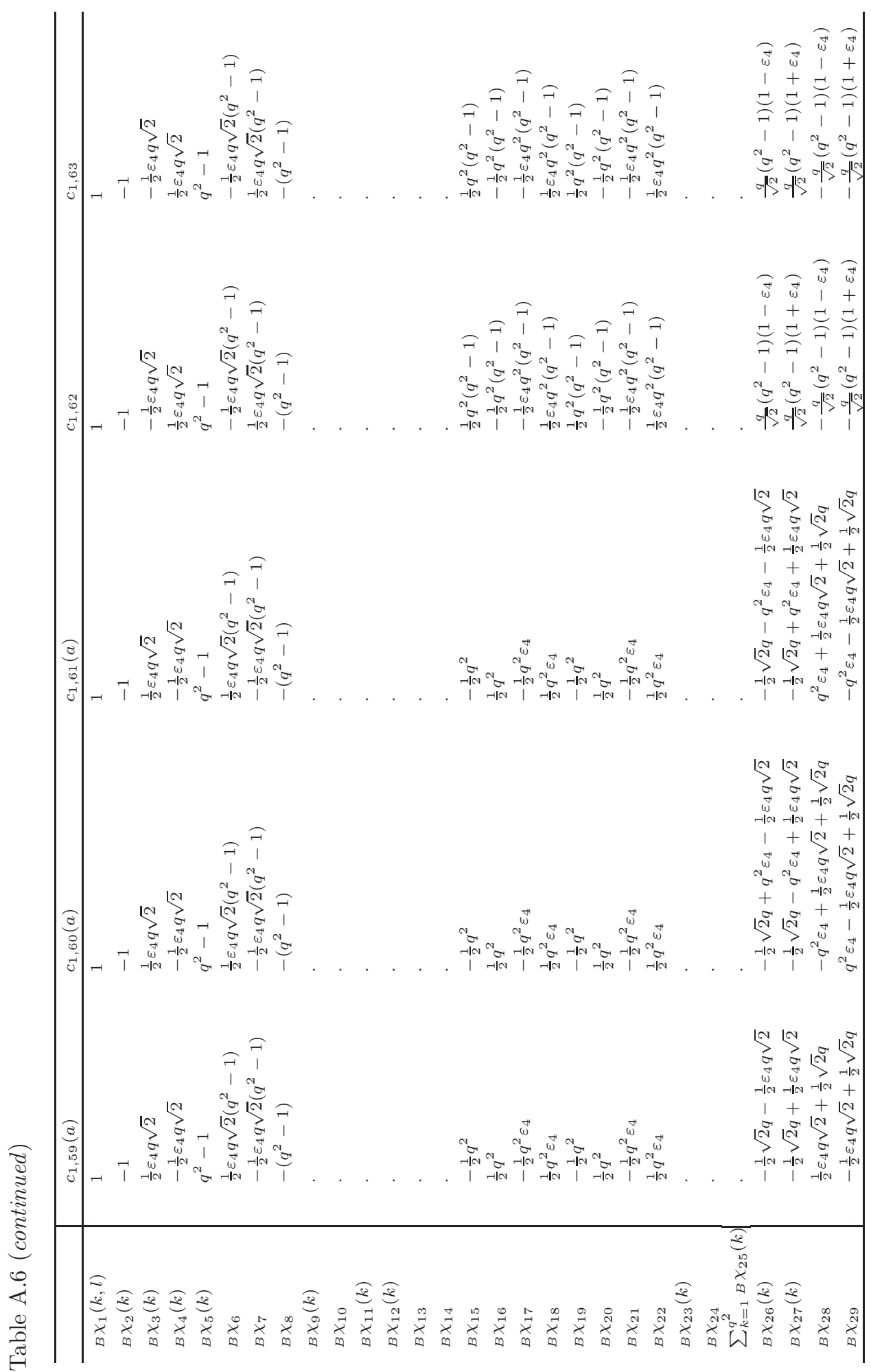


Character table of a Borel subgroup of the Ree groups ${ }^{2} F_{4}\left(q^{2}\right)$

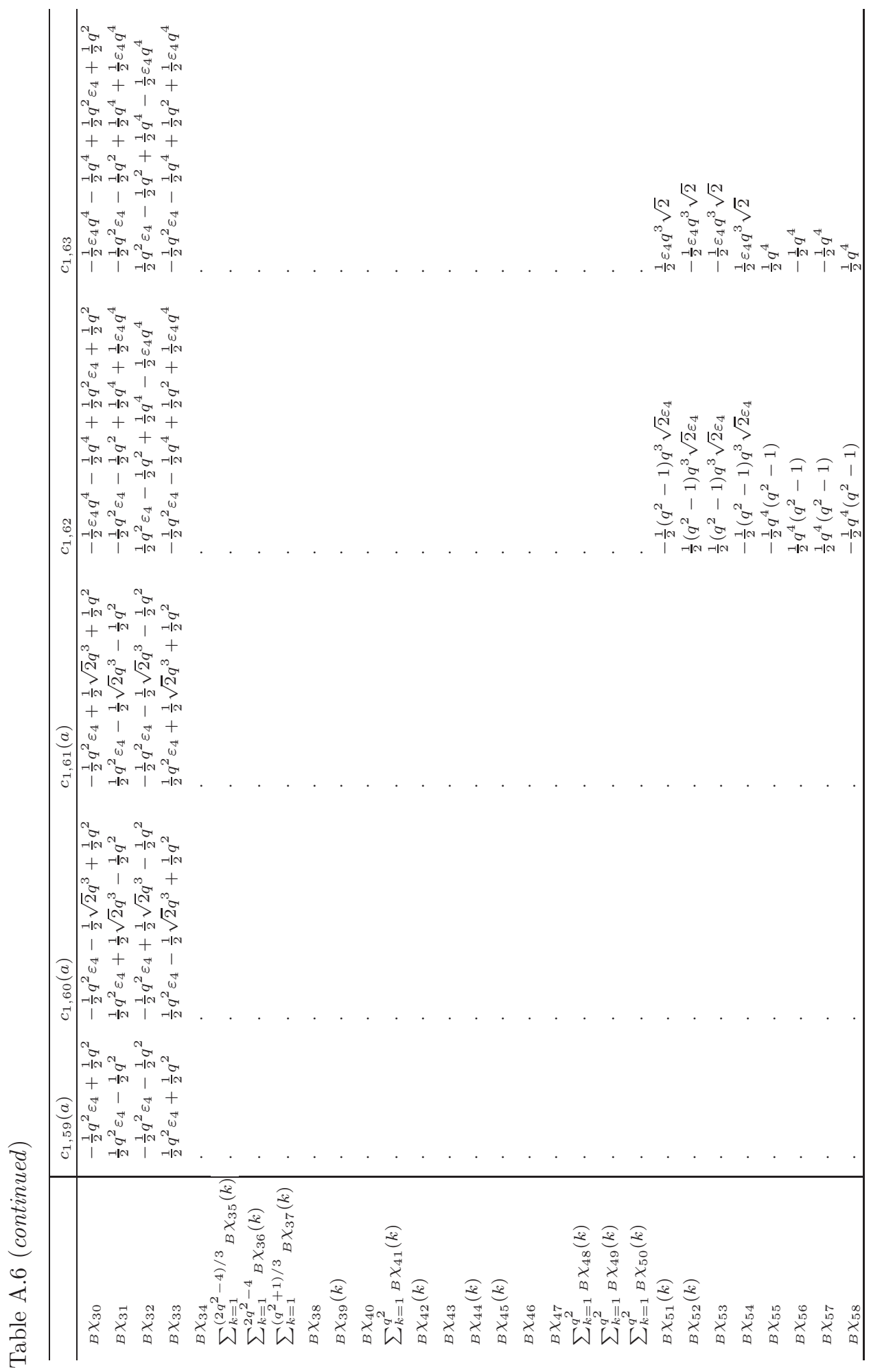


Character table of a Borel subgroup of the Ree groups ${ }^{2} F_{4}\left(q^{2}\right)$

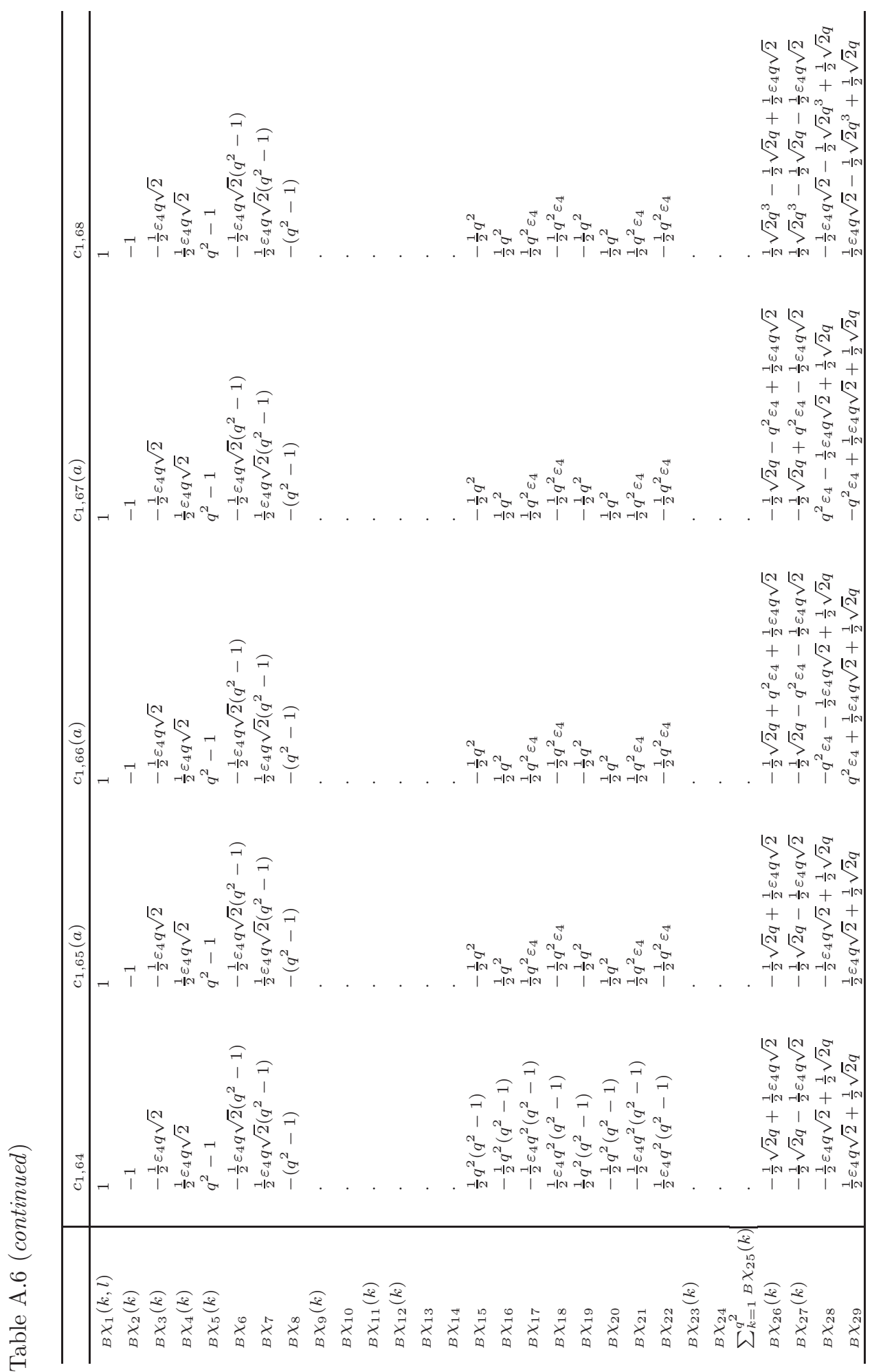


Character table of a Borel subgroup of the Ree groups ${ }^{2} F_{4}\left(q^{2}\right)$

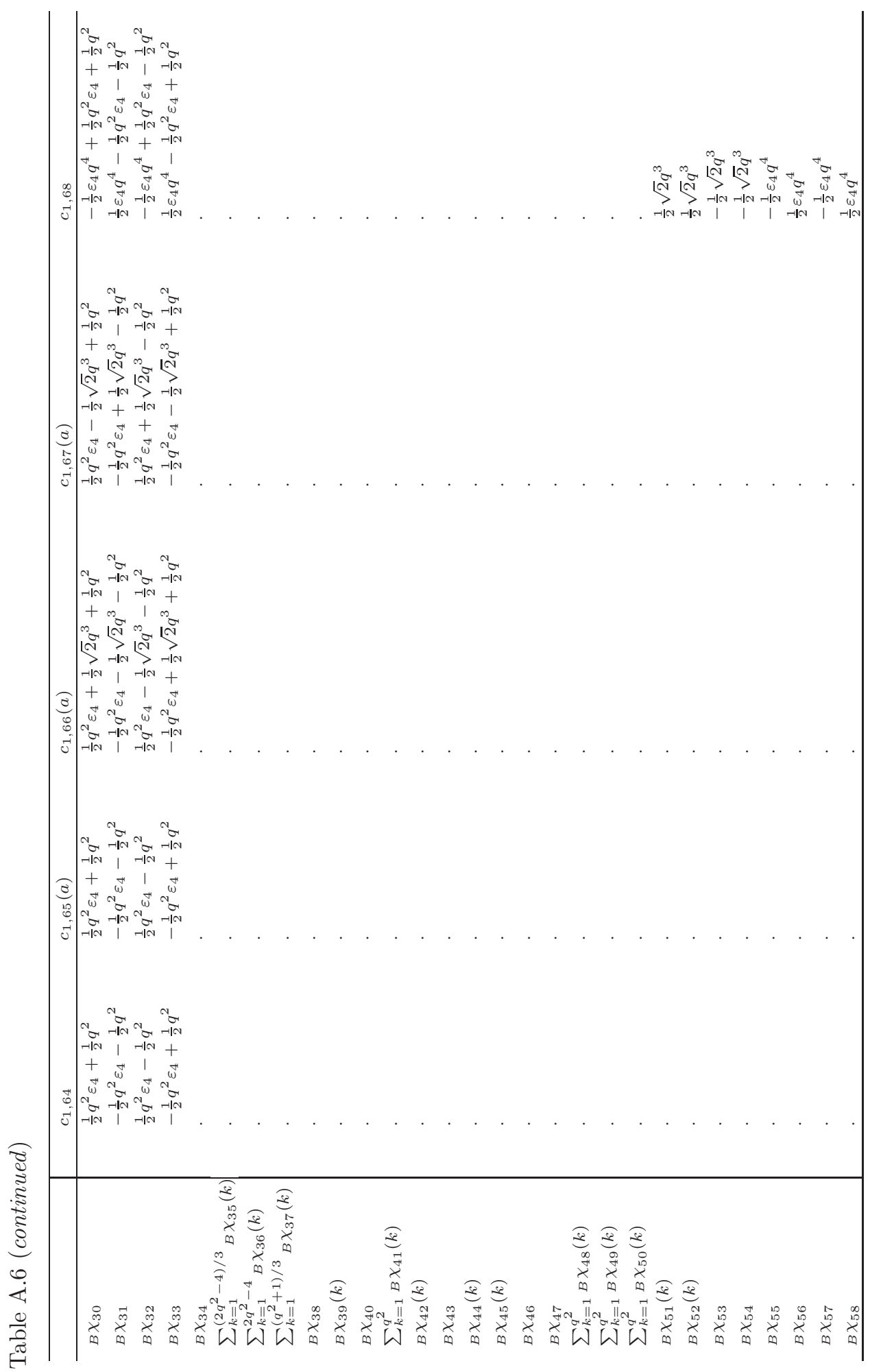


Character table of a Borel subgroup of the Ree groups ${ }^{2} F_{4}\left(q^{2}\right)$

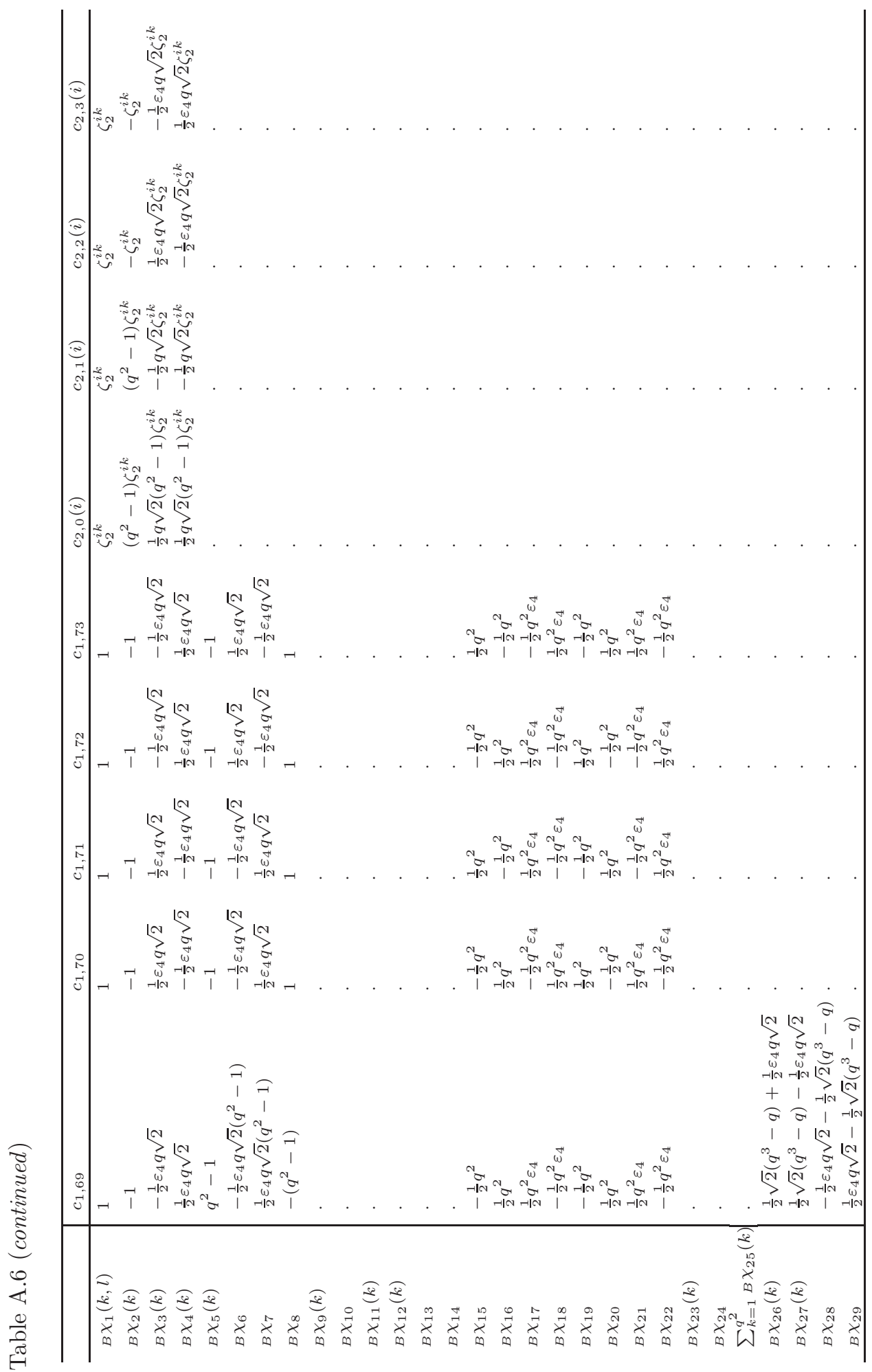


Character table of a Borel subgroup of the Ree groups ${ }^{2} F_{4}\left(q^{2}\right)$

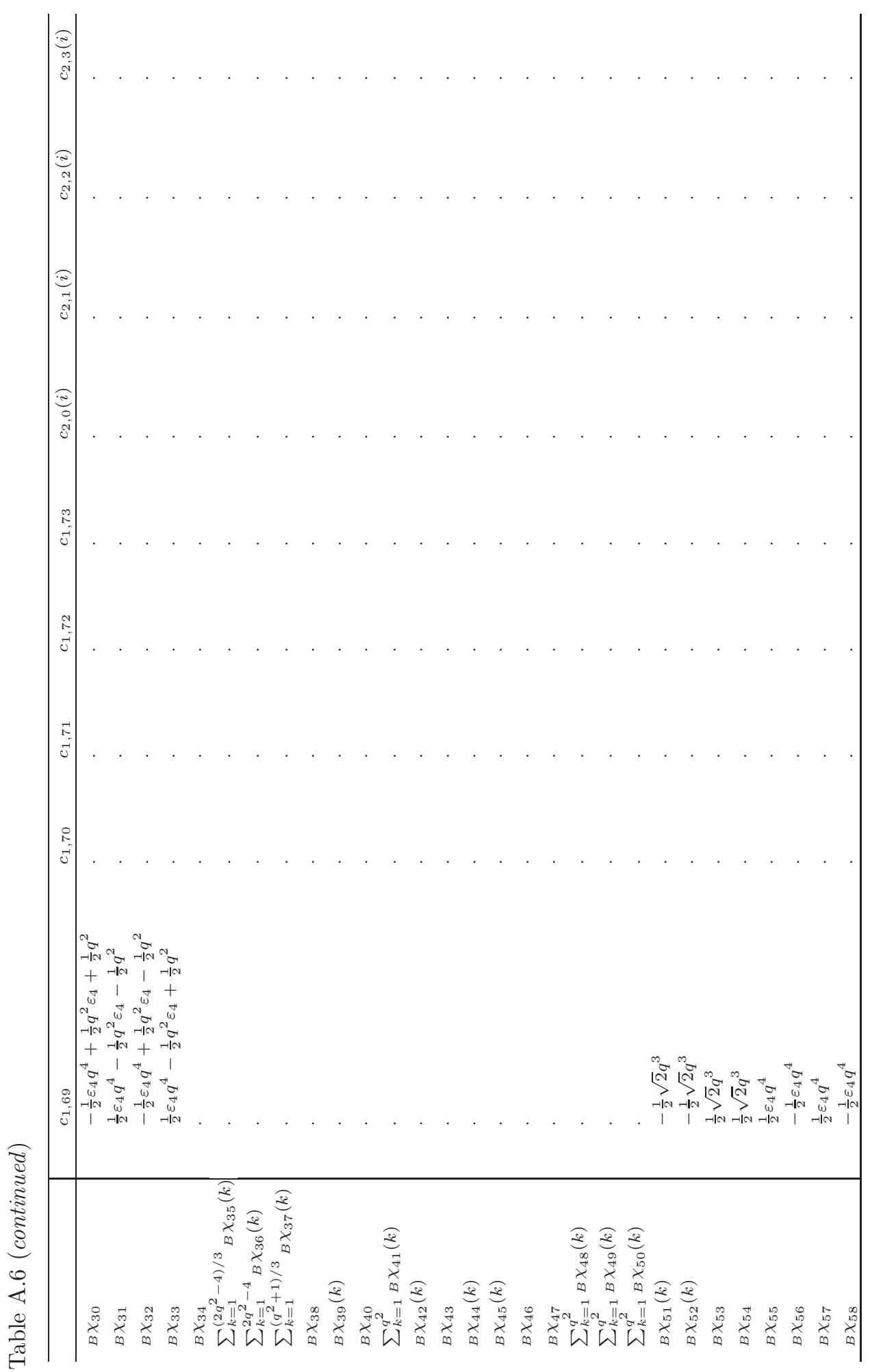


Character table of a Borel subgroup of the Ree groups ${ }^{2} F_{4}\left(q^{2}\right)$

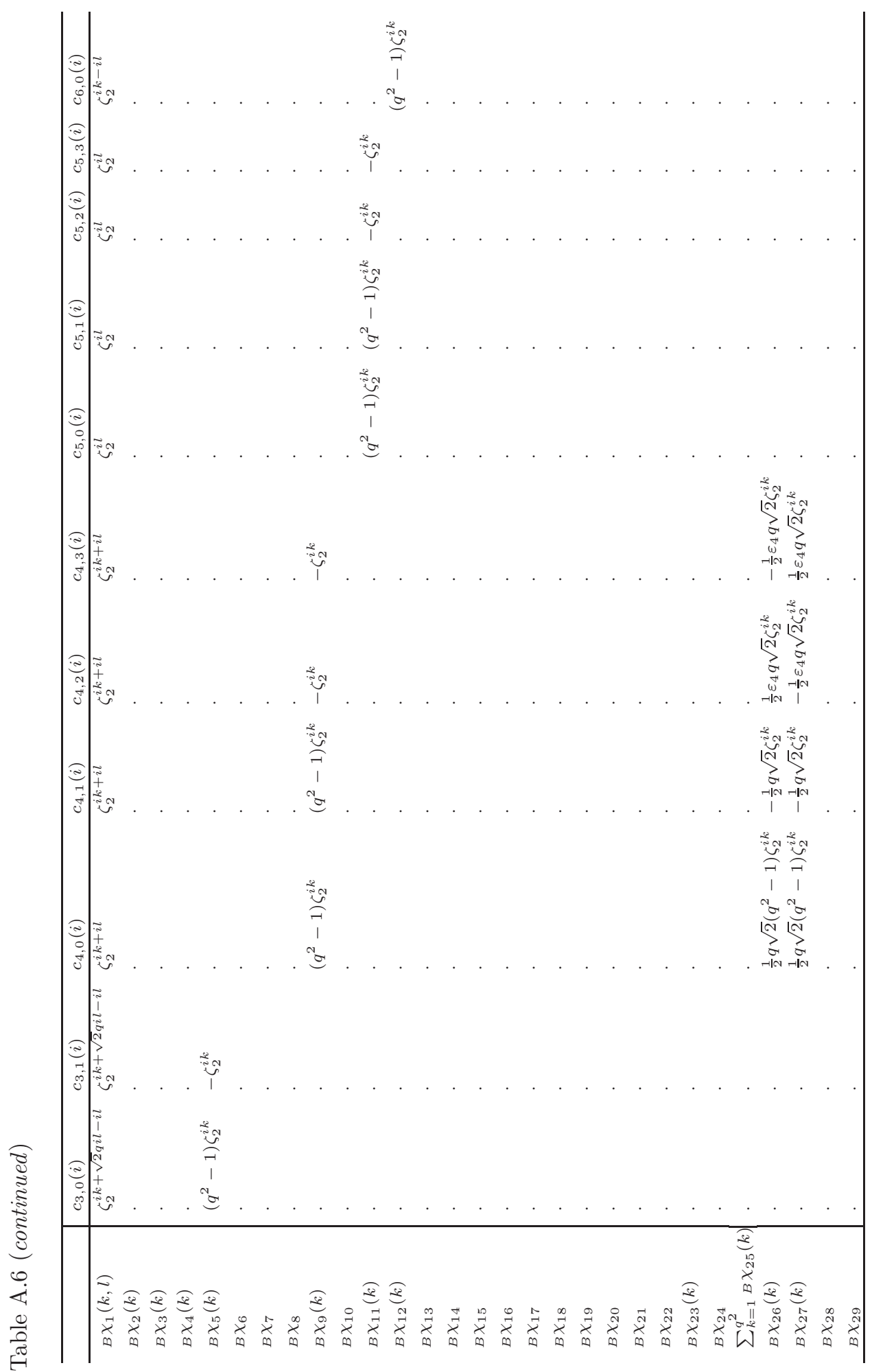


Character table of a Borel subgroup of the Ree groups ${ }^{2} F_{4}\left(q^{2}\right)$

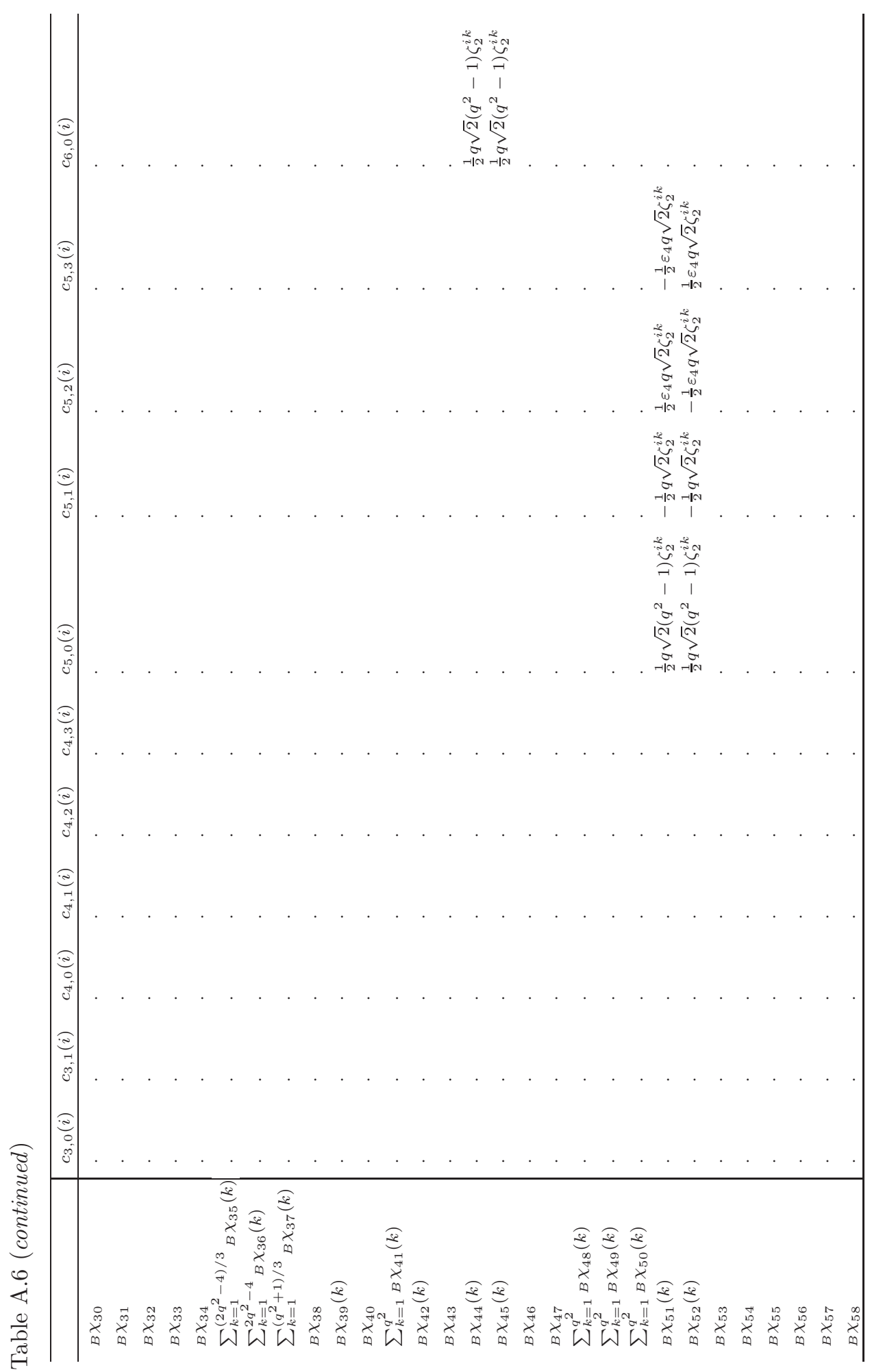


Character table of a Borel subgroup of the Ree groups ${ }^{2} F_{4}\left(q^{2}\right)$

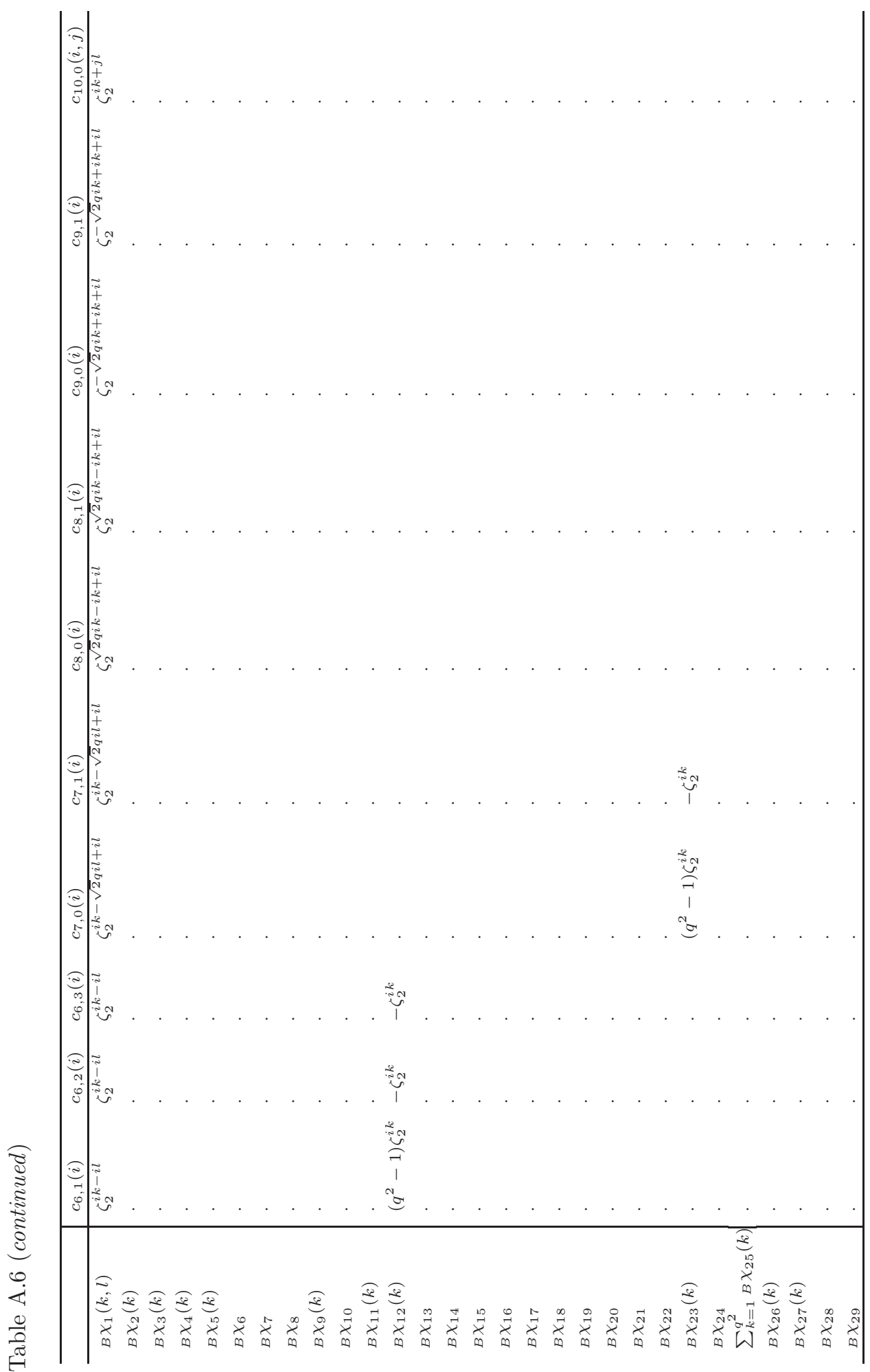


Character table of a Botel subgroup of the Ae groups ${ }^{2} F_{4}\left(q^{2}\right)$

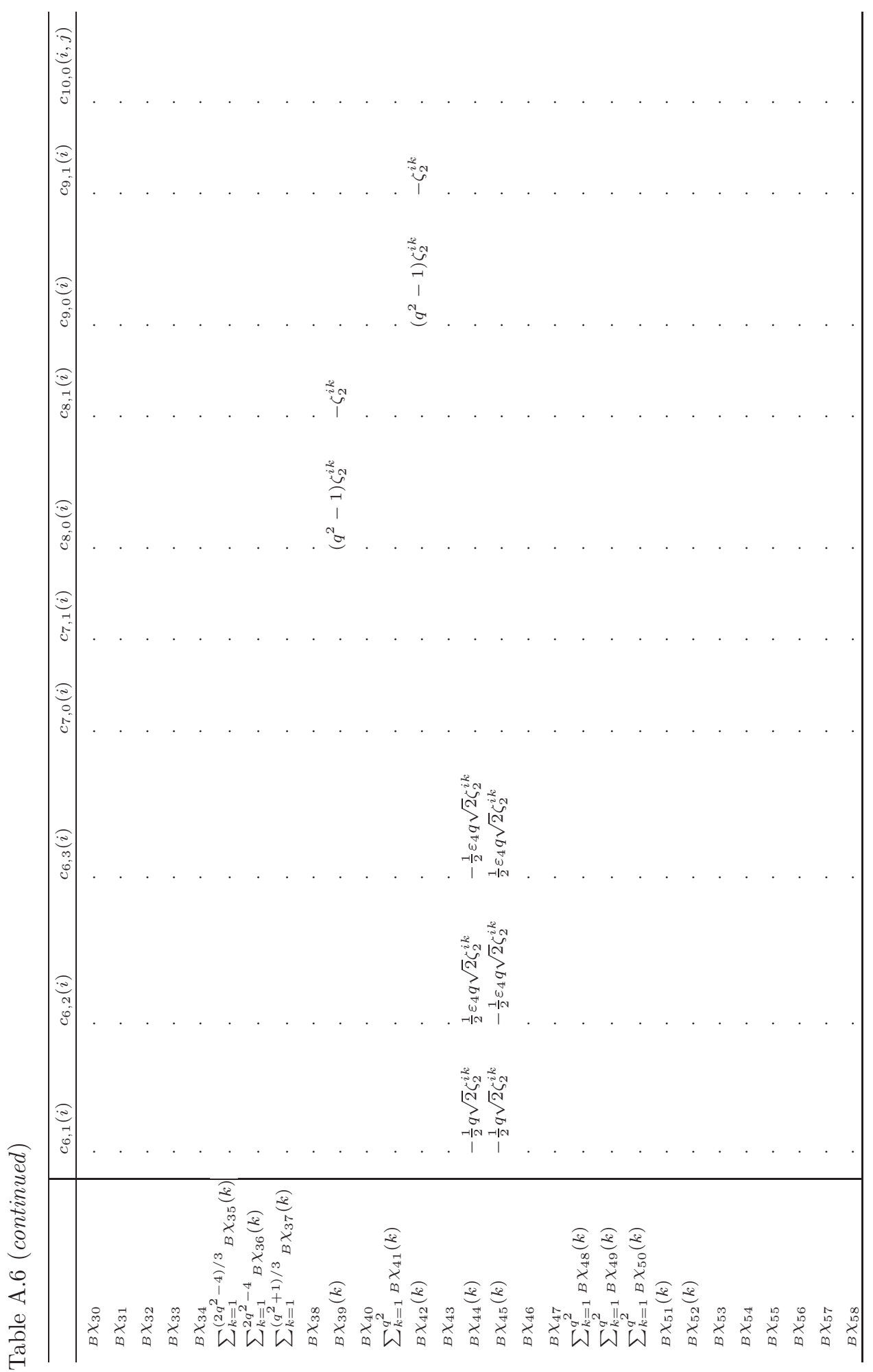

https://doi.org/10.1112/S1461157000000012 Published online by Caß̧pridge University Press 
Character table of a Borel subgroup of the Ree groups ${ }^{2} F_{4}\left(q^{2}\right)$

\section{References}

1. H.I. Blau and G.O. Michler, 'Modular representation theory of finite groups with T.I. Sylow p-subgroups', Trans. Amer. Math. Soc. 319 (1990) 417-468. 18

2. R. CARTER, Simple groups of Lie type (Wiley-Interscience, London, 1972). 2,4

3. R. CARTER, Finite groups of Lie type - Conjugacy classes and complex characters (Wiley-Interscience, Chichester, 1985). 4, 5, 6, 18

4. B.W. Char, K.O. Geddes, G.H. Gonnet, B.L. Leong, M.B. Monagan and S.M. WATT, Maple V, Language Reference Manual (Springer, 1991). 1

5. H. Enomoto and H. Yamada, 'The characters of the finite group $G_{2}\left(2^{n}\right)$ ', Japan. J. Math. 12 (1986) 325-377. 1, 16

6. The GAP Group, 'GAP - Groups, Algorithms, and Programming', Version 4.4.10 (2007), http://www.gap-system.org. 1

7. M. GECK, Verallgemeinerte Gelfand-Graev Charaktere und Zerlegungszahlen endlicher Gruppen von Lie-Typ, Dissertation, RWTH Aachen, 1990. 7

8. M. Geck, G. Hiss, F. LüBeck, G. Malle and G. Pfeiffer, 'CHEvie - A system for computing and processing generic character tables for finite groups of Lie type, Weyl groups and Hecke algebras', Appl. Algebra Engrg. Comm. Comput. 7 (1996) 175-210. 1

9. F. Himstedt, 'On the 2-decomposition numbers of Steinberg's triality groups ${ }^{3} D_{4}(q), q$ odd', J. Algebra 309 (2007) 569-593. 1

10. F. Himstedt and S.-C. Huang, 'Dade's invariant conjecture for Steinberg's triality groups ${ }^{3} D_{4}\left(2^{n}\right)$ in defining characteristic', J. Algebra 316 (2007) 802827. 17,18

11. B. HuPPERT, Endliche Gruppen I, Grundlagen der mathematischen Wissenschaften (Springer, 1967). 17

12. M. IsAACS, Character theory of finite groups (Dover, New York, 1976). 9, 10, $13,14,15,16,17,18$

13. M. IsaAcs, G. Malle and G. Navarro, 'A reduction theorem for the McKay conjecture', Invent. Math. 170 (2007) 33-101. 2, 17, 18

14. P. LANDROCK, Finite group algebras and their modules (Cambridge University Press, 1983). 16

15. F. LüBECK, Charaktertafeln für die Gruppen $C S p_{6}(q)$ mit ungeradem $q$ und $\mathrm{Sp}_{6}(q)$ mit geradem $q$, Dissertation, Universität Heidelberg, Germany, 1993. 5

16. G. Malle, 'Die unipotenten Charaktere von ${ }^{2} F_{4}\left(q^{2}\right)^{\prime}$, Comm. Alg. 18 (1990) 2361-2381. 1

17. R. REE, 'A Family of simple groups associated with the simple Lie algebra of type $\left(F_{4}\right)$ ', Amer. J. of Math. 83 (1961) 401-420. 2, 3

18. K. Shinoda, 'A characterization of odd order extensions of the Ree groups ${ }^{2} F_{4}(q)$ ', J. Fac. Sci. Univ. Tokyo Sect. I A Math. 22 (1975) 79-102. 3, 4, 5

19. K. Shinoda, 'The conjugacy classes of the finite Ree groups of type $\left(F_{4}\right)$ ', $J$. Fac. Sci. Univ. Tokyo Sect. I A Math. 22 (1975) 1-15. 2, 4, 5, 6, 7, 19 
Character table of a Borel subgroup of the Ree groups ${ }^{2} F_{4}\left(q^{2}\right)$

20. B. Szegedy, 'Characters of the Borel and Sylow subgroups of classical groups', J. Algebra 267 (2003) 130-136. 1

21. K. WAKI, 'A note on decomposition numbers of $G_{2}\left(2^{n}\right)$ ', J. Algebra $274(2004)$ 602-606. 1

Frank Himstedt himstedt@ma.tum.de

Technische Universität München

Zentrum Mathematik - M11

Boltzmannstr. 3

85748 Garching

Germany

Shih-chang Huang shua003@math.auckland.ac.nz

Department of Mathematics

University of Auckland

Private Bag 92019

Auckland

New Zealand 